\title{
Asymmetric Total Synthesis of C9'-epi-Sinefungin
}

Ludovic Decultot, Rocco L. Policarpo, Brandon Wright, Danny Huang, Matthew Shair

Submitted date: 17/05/2020 - Posted date: 19/05/2020

Licence: CC BY-NC-ND 4.0

Citation information: Decultot, Ludovic; Policarpo, Rocco L.; Wright, Brandon; Huang, Danny; Shair, Matthew (2020): Asymmetric Total Synthesis of C9'-epi-Sinefungin. ChemRxiv. Preprint.

https://doi.org/10.26434/chemrxiv.12318797.v1

The natural nucleoside (+)-sinefungin, structurally similar to cofactor S-adenosyl-L-methionine (SAM), inhibits various SAM-dependent methyltransferases (MTs). Access to sinefungin analogues could serve as the basis for the rational design of small-molecule methyltransferase inhibitors. We developed a route to the unnatural C9' epimer of sinefungin that employed a diastereoselective Overman rearrangement to install the key C6' amino stereocenter. The ability for late stage modification is highlighted, opening an avenue for the discovery of new MTs inhibitors.

File list (3)

EpiSinefungin_Decultot_Policarpo_ChemRxiv_2020.pdf (3.87 MiB) view on ChemRxiv - download file EpiSinefungin_Decultot_Policarpo_ChemRxiv_2020_SI.pdf (6.39 MiB) view on ChemRxiv • download file EpiSinefungin_Xray_Cpd12_ChemRxiv_2020.cif (402.81 KiB) view on ChemRxiv • download file 


\title{
Asymmetric Total Synthesis of C9'-epi-Sinefungin
}

\author{
Ludovic Decultot $^{\mathrm{a}, \grave{\ddagger}}$, Rocco L. Policarpo $^{\mathrm{b}, \dot{\ddagger}}$, Brandon A. Wright $^{\mathrm{c}}$, Danny Huang ${ }^{\mathrm{c}}$, Matthew D. Shair*. \\ Department of Chemistry and Chemical Biology, Harvard University, Cambridge, MA 02138, USA
}

ABSTRACT: The natural nucleoside (+)-sinefungin, structurally similar to cofactor $S$-adenosyl- $L$-methionine (SAM), inhibits various SAM-dependent methyltransferases (MTs). Access to sinefungin analogues could serve as the basis for the rational design of small-mole-

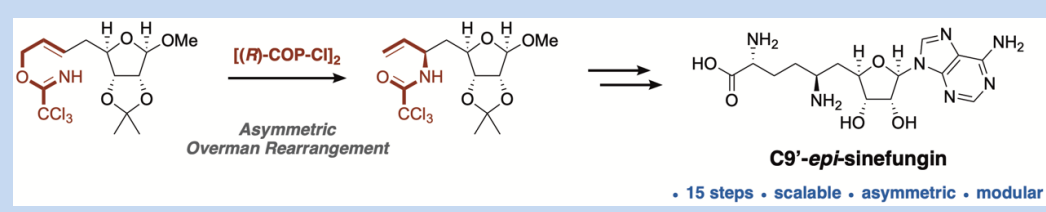
15 steps • scalable • asymmetric • modular cule methyltransferase inhibitors. We developed a route to the unnatural C9' epimer of sinefungin that employed a diastereoselective Overman rearrangement to install the key C6' amino stereocenter. The ability for late stage modification is highlighted, opening an avenue for the discovery of new MTs inhibitors.

$S$-Adenosyl- $L$-methionine (SAM)-dependent methyltransferases (MTs)"2 represent a large class of enzymes which use SAM (1, Figure 1), the second most ubiquitous cofactor, ${ }^{3}$ to methylate a wide variety of biomolecules, including DNA, proteins and small molecules, yielding $S$-adenosyl- $L$-homocysteine (SAH) as the byproduct. The methyl transfer from SAM to these various substrates plays a fundamental role in many biological processes including embryo development, ${ }^{4}$ epigenetic regulation of gene expression, ${ }^{, \%}$ cellular signaling, and metabolism. ${ }^{7}$ Methyltransferases have also emerged as disease-relevant targets ${ }^{\text {s.9 }}$ with abnormal expression implicated in neurological disorders, ${ }^{10.11}$ inflammatory diseases and a variety of cancers. ${ }^{1{ }^{2}{ }^{13}}$

(+)-Sinefungin (2), a naturally occurring nucleoside isolated from broths of Streptomyces griseolu ${ }^{14}$ and Streptomyces incarnatus, presents a structure highly homologous to the structure of SAM, with an amino methylene unit in place of the methyl sulfonium. Sinefungin is known to act as a competitive inhibitor of multiple SAM-dependent methyltransferase ${ }^{15{ }^{1 / 6}}$ and to exhibit antitumor, ${ }^{17}$ antiviral, ${ }^{18}{ }^{19}$ and antiparasitic activity..$^{20}$ Recently, sinefungin was shown to prevent myofibroblast activity in cell culture and in vivo, and to reduce myofibroblast activity in tumor stroma and metastasis burden in lungs in a mouse cancer model. ${ }^{21}$ Sinefungin has also emerged as a privileged scaffold in the development of potent and selective inhibitors of SAMdependent MTs. ${ }^{22{ }^{23} 3}$ Structural modification of sinefungin led to the development of a selective $\mathrm{N}$-alkyl inhibitor (3) of SETD2, ${ }^{24}$ a tumor-suppressing protein lysine methyltransferase (PKMT) whose aberrant activity is associated with multiple developmental pathologies and cancers. ${ }^{25}$ The elaboration of a cyclohexyl substituted analog of sinefungin (4) displaying inhibitory properties towards the PKMTs EHMT1 and EHMT2 with good selectivity was also reported. ${ }^{26}$

Inspired by the promising use of sinefungin and congeners to target SAM-dependent methyltransferases, herein we report the synthesis of C9'-epi-sinefungin. This study provides an efficient solution to the formation of the key C6' amine of the sinefungin scaffold. The reported synthesis is modular and amenable to the generation of sinefungin analogs to facilitate further investigation of sinefungin-derived MT inhibitors.<smiles>C[Sn](C)(CC[C@H](N)C(=O)O)C[C@H]1O[C@@H](n2cnc3c(N)ncnc32)[C@H](O)[C@@H]1O</smiles>

$S$-adenosyl-L-methionine (SAM, 1)<smiles>Nc1ncnc2c1ncn2[C@@H]1O[C@H](C[C@H](N)CC[C@H](N)C(=O)O)[C@@H](O)[C@H]1O</smiles>

sinefungin (2), pan-MT inhibitor<smiles>CCCN[C@@H](CC[C@H](N)C(=O)O)C[C@H]1O[C@@H](n2cnc3c(N)ncnc32)[C@H](O)[C@@H]1O</smiles>

$N$-propyl sinefungin (3), SETD2 inhibitor

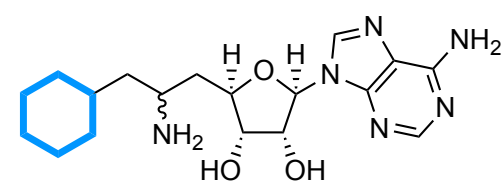

cyclohexyl sinefungin analog (4), EHMT1/2 inhibitor

Figure 1. Structures of SAM (1), sinefungin (2) and analogs $(\mathbf{3}, \mathbf{4})$.

The biochemical activity of sinefungin have made it an attractive target for the synthetic community (Figure 2). Since its first synthesis in 1982 by Mock and co-workers, ${ }^{27}$ existing literature has highlighted the C6' stereocenter as the main synthetic challenge en route to sinefungin. This amine was either accessed in a racemic fashion to give a mixture of C6' epimers most often separable by chromatographic methods, ${ }^{28}{ }^{29930}$ or through stereoselective transformations. Such examples include the alkylation of a chiral imide auxiliary by Ghosh et al...1 or the diastereose- 
lective reduction of a ketone at C6' by Rapoport and colleagues.32 In all cases, multiple functional group interconversions were required to elaborate the alkyl chain of the nucleoside natural product, limiting the tractability of these approaches for the efficient generation of sinefungin and potential analogs. Taken together, these results underline the remaining need for a robust solution to the formation of the C6' amino stereocenter and for a modular synthesis if one aims to rapidly assemble such molecules.

$$
\begin{aligned}
& \text { condensation }
\end{aligned}
$$

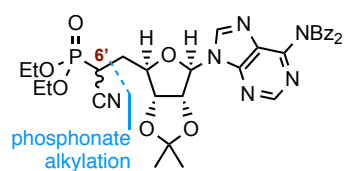

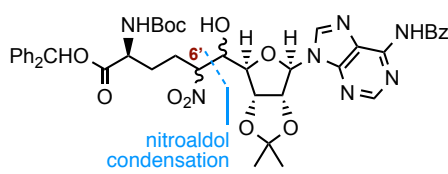

$$
\begin{aligned}
& \text { d.r. }{ }_{92: 8}^{t \text {-BuOO }} \\
& \text { addition }
\end{aligned}
$$

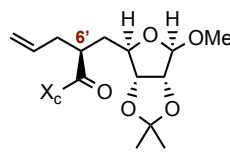

$$
\begin{aligned}
& \text { d.r. } 96: 4 \underbrace{\text { (O) }}_{\mathrm{N}_{3}}
\end{aligned}
$$

Figure 2. Previously reported syntheses of (+)-sinefungin.

Our synthesis of C9'-epi-sinefungin (5) proceeded through key intermediate trichloroacetamide 7 (Scheme 1). Retrosynthetic analysis suggested that the adenine fragment could be installed by means of a Vorbrüggen glycosylation from triacetate $\mathbf{6}$. The amino acid could derive from an aldehyde by a HornerWadsworth-Emmons olefination / rhodium-catalyzed asymmetric hydrogenation sequence. This strategy proved successful in our work on the development of high-affinity inhibitors of nicotinamide $N$-methyltransferase (NNMT). ${ }^{.3}$ Next, we envisioned that the key C6' stereocenter could be introduced by a COP-catalyzed $(\mathrm{COP}=$ cobalt oxazoline palladacycle $)$ asymmetric Overman rearrangement of trichloroacetimidate 8 to provide the $(S)$-amine present in the target product.
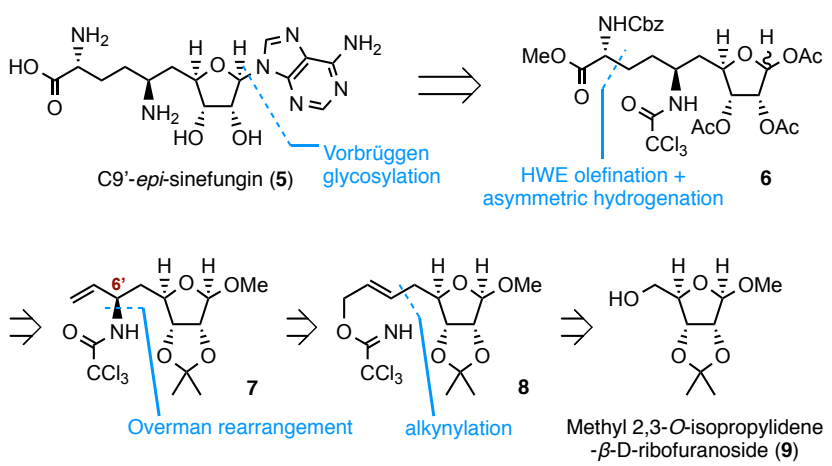

Scheme 1. Retrosynthetic analysis. HWE = Horner-Wadsworth-Emmons .

Trichloroacetamide 7 was prepared in 6 scalable steps from acetonide 9, commercially available or readily accessible from Dribose $^{34}$ (Scheme 2). Triflation of acetonide 9 and subsequent alkynylation upon treatment with the lithium acetylide derived from $t$-butyldimethyl(2-propynyloxy)silane yielded silyl protected propargyl alcohol $\mathbf{1 0}$ in quantitative yield. Cleavage of the TBS protecting group followed by reduction to the $E$-alkene with $\mathrm{LiAlH}_{4}$ afforded known allylic alcohol $11^{\text {s!b }}$ in $63 \%$ yield over 2 steps. Alcohol 11 was converted to trichloroacetimidate $\mathbf{8}$ in the presence of trichloroacetonitrile and in excellent yield, giving access to the precursor required for the envisioned Overman rearrangement.

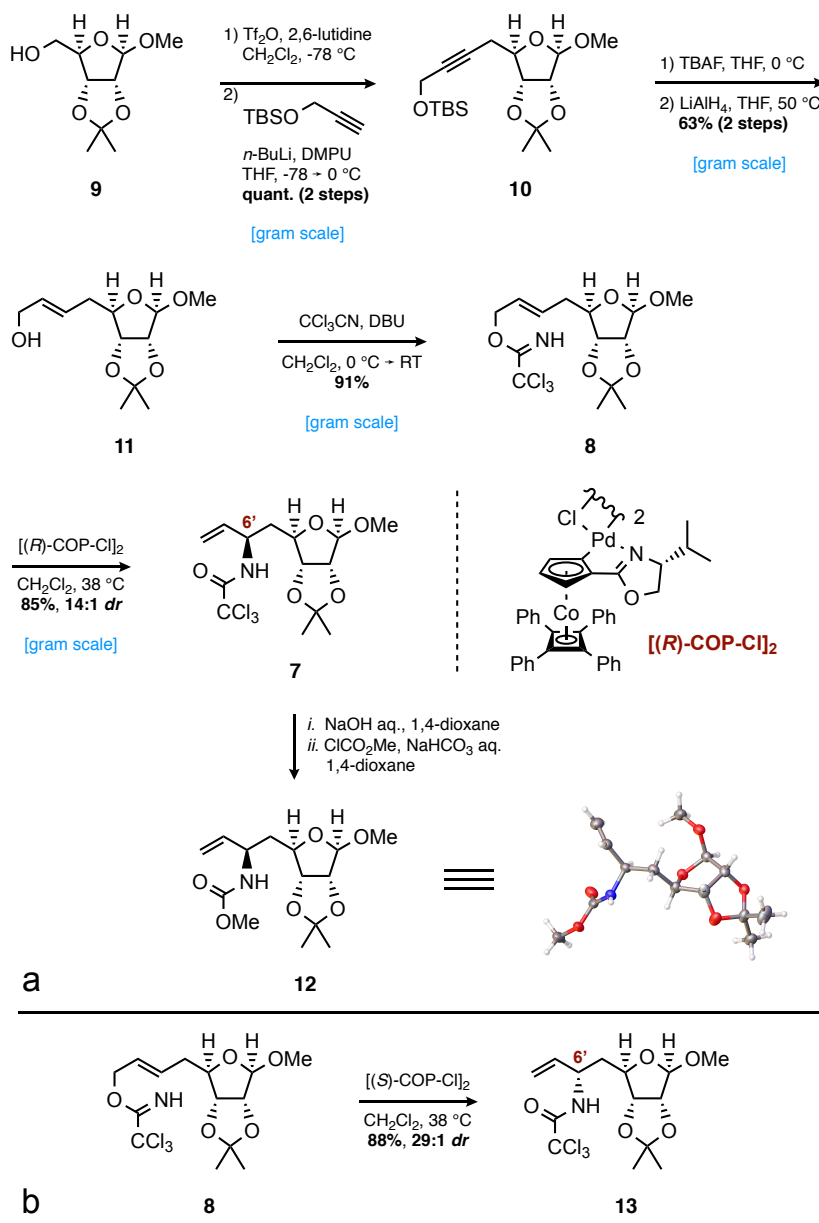

Scheme 2. (a) Synthesis of trichloroacetamide 7. (b) Overman rearrangement towards C6' $(S)$-epimer 13. COP = cobalt oxazoline palladacycle. 
Treatment of $E$-allylic trichloroacetimidate 8 in $\mathrm{CH}_{2} \mathrm{Cl}_{2}$ with Overman's $[(R)-\mathrm{COP}-\mathrm{Cl}]_{2^{35}}$ effected the desired [3,3]-sigmatropic rearrangement, delivering allylic trichloroacetamide 7 in $85 \%$ yield with a 14:1 $d r$. The minor diastereomer could be separated under standard silica gel chromatography conditions. While the extended reaction time proved necessary to reach the observed conversion, no erosion of $d r$ was observed over the course of the reaction. The $[(R)-\mathrm{COP}-\mathrm{Cl}]_{2}$ catalyst was prepared in one step from $[(R)-\mathrm{COP}-\mathrm{OAc}]_{2}$ according to a procedure from Overman and co-workers. ${ }^{36}$ Use of the related $(R)$-COPhfacac catalyst (hfacac $=$ hexafluoroacetylacetonate) did not prove beneficial and a slower conversion was observed with a comparable $d r$. An increased reaction temperature $\left(50{ }^{\circ} \mathrm{C}\right)$ in 1,2-DCE did not improve the outcome, with similar yield and $d r$ noted. Derivatization of compound 7 to the more crystalline carbamate 12 allowed for a small-molecule X-ray crystal structure to be obtained, confirming the $(R)$ configuration of the $\mathrm{C} 6$ ' stereocenter. Interestingly, when $[(S)-\mathrm{COP}-\mathrm{Cl}]_{2}$ was used to catalyze the rearrangement, the C6' (S)-trichloroacetamide 13 was obtained in high diastereoselectivity to give the $(S)$-amide epimer in 29:1 $d r$, thus demonstrating that the stereochemical outcome of this rearrangement is largely under catalyst control. This result also suggests some degree of substrate control, but the catalyst choice largely overcomes this inherent substrate bias.

With this diastereoselective rearrangement providing an efficient solution to the installation of the stereocenter at C6', we turned our attention to the amino acid moiety (Scheme 3 ). Terminal alkene 7 was converted in good yield to aldehyde 14 under Wacker-Tsuji oxidation conditions using $t$-butyl nitrile as an organic redox cocatalyst. ${ }^{37}$ It is worth noting that aldehyde $\mathbf{1 4}$ could represent a powerful handle to enable the synthesis of various analogs aimed at replacing the amino acid moiety. A Horner-Wadsworth-Emmons olefination with Cbz- $\alpha$-phosphonoglycine trimethyl ester yielded enamide 15. The C9' amino stereocenter was set using a rhodium-catalyzed asymmetric hydrogenation with $(S, S)$-Ph-BPE-Rh $\left(\mathrm{BF}_{4}\right.$ salt), a Burktype 1,2-bis(phospholano)ethane (BPE) ligand, ${ }^{38}{ }^{39}$ providing $(R)$-carbamate $\mathbf{1 6}$ as a single diastereomer and with no observed side reactions.

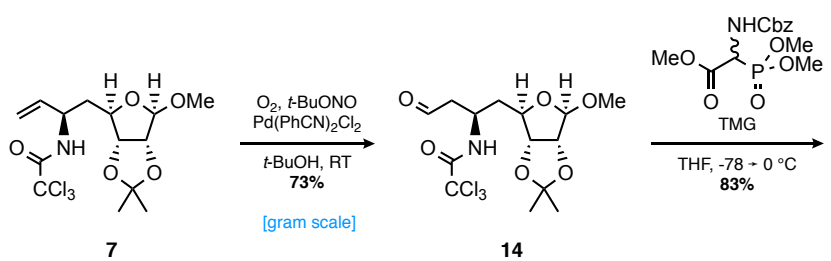

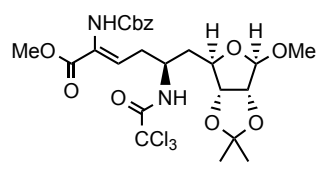

15

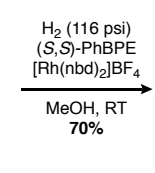

16

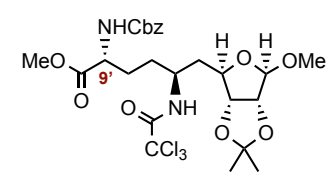

Scheme 3. Elaboration of the amino acid moiety. TMG $=1,1,3,3$-tetramethylguanidine; $\mathrm{BPE}=$ bis(phospholano)ethane; $\mathrm{nbd}=$ norbornadiene .

With the $\mathrm{C} 5$ ' $-\mathrm{C} 10$ ' amino acid chain elaborated, the installation of the adenine moiety and subsequent global deprotection were undertaken (Scheme 4). To this end, acetonide $\mathbf{1 6}$ was converted in two steps into triacetate 6, as a 2:1 mixture of anomers at $\mathrm{C} 1$ ' (by ${ }^{1} \mathrm{H} \mathrm{NMR}$ ). Vorbrüggen glycosylation promoted by

2,6-di-tert-butyl-4-methylpyridinium triflate ${ }^{40}$ provided nucleoside $\mathbf{1 7}$ in $79 \%$ yield and as a single diastereomer.

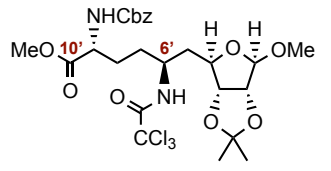

16

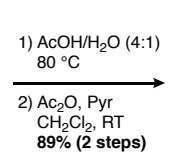

$89 \%$ (2 steps)

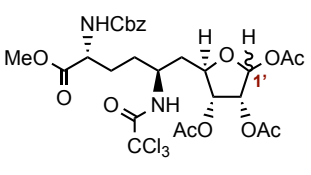

6

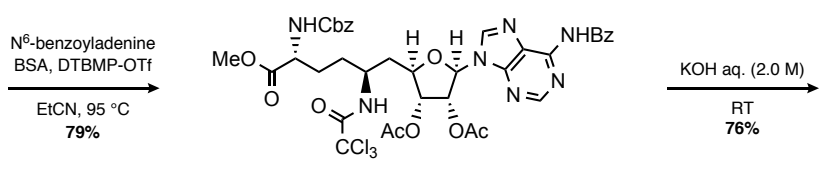

17

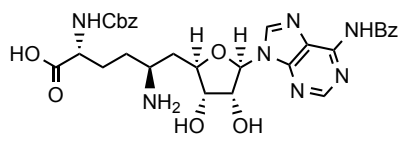

18
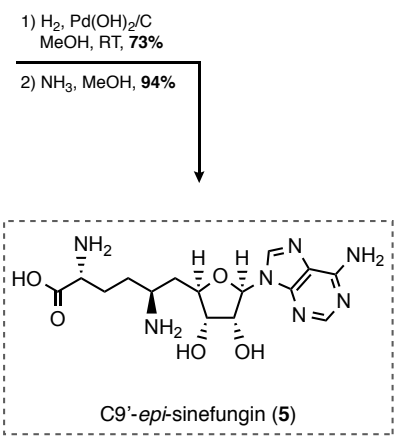

Scheme 4. Installation of the nucleobase and completion of the total synthesis. BSA $=N, O$-bis(trimethylsilyl)acetamide; DTBMP-OTf $=2,6$-ditert-butyl-4-methylpyridinium triflate.

With the entire carbon framework assembled, we finally examined the removal of the various protecting groups. We were particularly interested in implementing conditions which would selectively deprotect the C6' amine over the C9' one, therefore opening a practical avenue for the generation of sinefungin analogs at this position, similar to the work of the Luo group and their report on $N$-propyl sinefungin. ${ }^{24}$ Treatment of trichloroacetamide 17 with aqueous potassium hydroxide delivered amine $\mathbf{1 8}$ in good yield and left the C9'-Cbz protected amine intact. Finally, cleavage of the remaining $\mathrm{Cbz}$ and $\mathrm{Bz}$ protecting groups by hydrogenolysis and subsequent addition of methanolic ammonia completed the synthesis of C9'-epi-sinefungin in a total of 15 steps with an overall yield of $7.6 \%$.

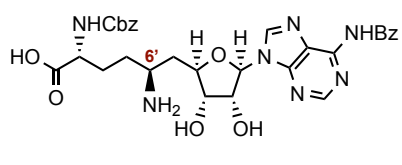

18
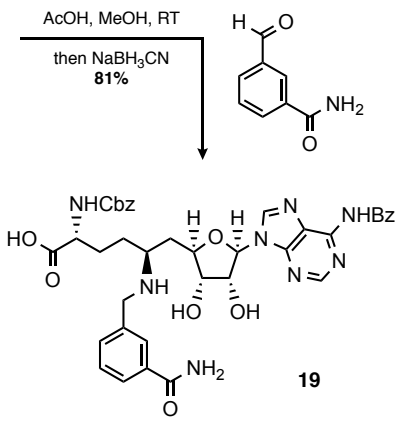

Scheme 5. Functionalization of the C6' amine.

Finally, to illustrate the utility of this deprotection sequence, we subjected amine 18 to reductive amination conditions with 3formyl-benzamide (Scheme 5). Benzamide 19, directly inspired 
by the structure of our high-affinity alkynyl inhibitor of nicotinamide $N$-methyltransferase ${ }^{33}$ and related inhibitors, ${ }^{41}$ was obtained in $81 \%$ yield, highlighting our ability to functionalize the C6' amine at a late stage.

In conclusion, an efficient asymmetric Overman rearrangement was developed to install the C6' amine present in the sinefungin scaffold, providing a tractable solution to this key challenge and avoiding multiple functional group interconversions as well as separation of diastereomers. The study was concluded by the synthesis of C9'-epi-sinefungin, featuring an asymmetric rhodium-catalyzed hydrogenation to form the amino acid stereocenter. The synthesis was designed to be modular and amenable to the elaboration of sinefungin analogs for further biological evaluation, as demonstrated by an illustrative functionalization of the C6' amine.

\section{ASSOCIATED CONTENT}

\section{Supporting Information}

Synthetic schemes, detailed experimental protocols, NMR spectra (PDF)

Small-molecule X-ray crystallography data for 12 (CIF)

\section{AUTHOR INFORMATION}

\section{Corresponding Author}

*E-mail: shair@,chemistry.harvard.edu. Phone: 617-495-5008.

\section{Present Addresses}

a The Broad Institute of MIT and Harvard, Center for the Development of Therapeutics, 415 Main Street, Cambridge, Massachusetts 02142 , United States

b Incyte Corporation, 1801 Augustine Cut-off, Wilmington, Delaware 19803, United States

c Department of Chemistry, University of California, Berkeley, California 94720, United States

\section{Author Contributions}

The manuscript was written through contributions of all authors. / All authors have given approval to the final version of the manuscript. / \$These authors contributed equally.

\section{Notes}

The authors declare no competing financial interests.

\section{ACKNOWLEDGMENT}

The authors thank Dr. Shao-Liang Zheng for assistance with small molecule X-ray crystallography and Jennifer X Wang for assistance with analytical structural characterization. We thank the National Science Foundation for funding Rocco L. Policarpo (NSF Graduate Research Fellowship under Grant No. DGE1144152) and the Harvard College Research Program for providing funding to Danny Huang and Brandon A. Wright.

\section{REFERENCES}

Klimašauskas, S.; Weinhold, E. A new tool for biotechnology: AdoMet-dependent methyltransferases. Trends Biotechnol. 2007, 25, 99-104.

'Struck, A.-W.; Thompson, M. L.; Wong, L. S.; Micklefield, J. SAdenosyl-methionine-dependent methyltransferases: highly versatile enzymes in biocatalysis, biosynthesis and other biotechnological applications. ChemBioChem 2012, 13, 2642-2655.
Fontecave, M.; Atta, M.; Mulliez, E. S-Adenosylmethionine: nothing goes to waste. Trends Biochem. Sci. 2004, 29, 243-249.

· Tachibana, M.; Sugimoto, K.; Nozaki, M.; Ueda, J.; Ohta, T.; Ohki, M.; Fukuda, M.; Takeda, N.; Niida, H.; Kato, H.; Shinkai, Y. G9a histone methyltransferase plays a dominant role in euchromatic histone $\mathrm{H} 3$ lysine 9 methylation and is essential for early embryogenesis. Gene Dev. 2002, 16, 1779-1791.

Kouzarides, T. Chromatin modifications and their function. Cell 2007, 128, 693-705.

- Bedford, M. T.; Clarke, S. G. Protein arginine methylation in mammals: who, what, and why. Mol. Cell 2009, 33, 1-13.

Pissios, P. Nicotinamide N-methyltransferase: more than a vitamin B3 clearance enzyme. Trends Endocrinol. Metab. 2017, 28, 340-353.

* Robertson, K. D. DNA methylation and human disease. Nat. Rev. Genet. 2005, 6, 597-610.

' Copeland, R. A.; Solomon, M. E.; Richon, V. M. Protein methyltransferases as a target class for drug discovery. Nat. Rev. Drug Disc. 2009, 8, 724-32.

Kleefstra, T.; Smidt, M.; Banning, M. J.; Oudakker, A. R.; Van Esch, H.; de Brouwer, A. P.; Nillesen, W.; Sistermans, E. A.; Hamel, B. C.; de Bruijn, D.; Fryns, J. P.; Yntema, H. G.; Brunner, H. G.; de Vries, B. B.; van Bokhoven, H. Disruption of the gene euchromatin histone methyl transferase1 (Eu-HMTase1) is associated with the $9 \mathrm{q} 34$ subtelomeric deletion syndrome. J. Med. Genet. 2005, 42, 299-306.

Tsankova, N.; Renthal, W.; Kumar, A.; Nestler, E. J. Epigenetic regulation in psychiatric disorders. Nat. Rev. Neurosci. 2007, 8, 355367.

Simon, J. A.; Lange, C. A. Roles of the EZH2 histone methyltransferase in cancer epigenetics. Mutat. Res. 2008, 647, 21-29.

Eckert, M. A.; Coscia, F.; Chryplewicz, A.; Chang, J. W.; Hernandez, K. M.; Pan, S.; Tienda, S. M.; Nahotko, D. A.; Li, G.; Blaženović, I.; Lastra, R. R.; Curtis, M.; Yamada, S. D.; Perets, R.; McGregor, S. M.; Andrade, J.; Fiehn, O.; Moellering, R. E.; Mann, M.; Lengyel, E. Proteomics reveals NNMT as a master metabolic regulator of cancerassociated fibroblasts. Nature 2019, 569, 723-728.

"Hamil, R. L.; Hoehn, M. M. A9145, a new adenine-containing antifungal antibiotic: I. discovery and isolation. J. Antibiot. 1973, 26, 463465 .

Borchardt, R. T.; Eiden, L. E.; Wu, B.; Rutledge, C. O. Sinefungin, a potent inhibitor of $S$-adenosylmethionine: protein $O$-methyltransferase. Biochem. Biophys. Res. Commun. 1979, 89, 919-924.

${ }^{16}$ Barbes, C.; Sanchez, J.; Yebra, M. J.; Robert-Gero, M.; Hardisson, C. Effects of sinefungin and $S$-adenosylhomocysteine on DNA and protein methyltransferases from Streptomyces and other bacteria. FEMS Microbiol. Lett. 1990, 69, 239-243.

Zingg, J. M.; Shen, J. C.; Yang, A. S.; Rapoport, H.; Jones, P. A. Methylation inhibitors can increase the rate of cytosine deamination by (cytosine-5)-DNA methyltransferase. Nucleic Acids Res. 1996, 24, 3267-3275

${ }^{1 s}$ Pugh, C. S. G.; Borchardt, R. T.; Stone, H. O. Sinefungin, a potent inhibitor of virion mRNA(guanine-7-)-methyltransferase, mRNA(nucleoside-2'-)-methyltransferase, and viral multiplication. J. Biol. Chem. 1978, 253, 4075-4077.

"Pugh, C. S. G.; Borchardt, R. T. Effects of $S$-adenosylhomocysteine analogs on vaccinia viral mRNA synthesis and methylation. Biochemistry 1982, 21, 1535-1541.

${ }^{20}$ Dube, D. K.; Mpimbaza, G.; Allison, A. C.; Lederer, E.; Rovis, L. Antitrypanosomal activity of sinefungin. Am. J. Trop. Med. Hyg. 1983, 32, 31-33.

Sala, L.; Franco-Valls, H.; Stanisavljevic, J.; Curto, J.; Vergés, J.; Peña, R.; Duch, P.; Alcaraz, J.; García de Herreros, A.; Baulida, J. Abrogation of myofibroblast activities in metastasis and fibrosis by methyltransferase inhibition. Int. J. Cancer 2019, 145, 3064-3077.

Zhang, J.; Zheng, Y. G. SAM/SAH analogs as versatile tools for SAM-dependent methyltransferases. ACS Chem. Biol. 2016, 11, 583597.

${ }^{2}$ Tao, Z.; Cao, R.; Yan, Y.; Huang, G.; Lv, K.; Li, W.; Geng, Y.; Zhao, L.; Wang, A.; He, Q.; Yang, J.; Fan, S.; Huang, M.; Guo, H.; Zhong, W.; Liu, M. Design, synthesis and in vitro anti-Zika virus evaluation of novel sinefungin derivatives. Eur. J. Med. Chem. 2018, 157, 994-1004. 
"Zheng, W.; Ibáñez, G.; Wu, H.; Blum, G.; Zeng, H.; Dong, A.; Li, F.; Hajian, T.; Allali-Hassani, A.; Amaya, M. F.; Siarheyeva, A.; Yu, W.; Brown, P. J.; Schapira, M.; Vedadi, M.; Min, J.; Luo, M. Sinefungin derivatives as inhibitors and structure probes of protein lysine methyltransferase SETD2. J. Am. Chem. Soc. 2012, 134, 18004-18014.

"s (a) Al Sarakbi, W.; Sasi, W.; Jiang, W. G.; Roberts, T.; Newbold, R. F.; Mokbel, K. The mRNA expression of SETD2 in human breast cancer: correlation with clinico-pathological parameters. BMC Cancer 2009, 9, 290-296. (b) Licht, J. D. SETD2: a complex role in blood malignancy. Blood 2017, 130, 2576-2578. (c) Mar, B. G.; Chu, S. H.; Kahn, J. D.; Krivtsov, A. V.; Koche, R.; Castellano, C. A.; Kotlier, J. L.; Zon, R. L.; McConkey, M. E.; Chabon, J.; Chappell, R.; Grauman, P. V.; Hsieh, J. J.; Armstrong, S. A.; Ebert, B. L. SETD2 alterations impair DNA damage recognition and lead to resistance to chemotherapy in leukemia. Blood 2017, 130, 2631-2641. (d) Skucha, A.; Ebner, J.; Grebien, F. Roles of SETD2 in leukemia-transcription, DNAdamage, and beyond. Int. J. Mol. Sci. 2019, 20, 1029-1040.

${ }^{26}$ (a) Devkota, K.; Lohse, B.; Liu, Q.; Wang, M.-W.; Stærk, D.; Berthelsen, J.; Clausen, R. P. Analogues of the natural product sinefungin as inhibitors of EHMT1 and EHMT2. ACS Med. Chem. Lett. 2014, 5, 293-297. (b) Liu, Q.; Cai, X.; Yang, D.; Chen, Y.; Wang, Y.; Shao, L.; Wang, M.-W. Cycloalkane analogues of sinefungin as EHMT1/2 inhibitors. Bioorg. Med. Chem. 2017, 25, 4579-4594.

${ }^{27}$ Mock, G. A.; Moffatt, J. G. An approach to the total synthesis of sinefungin. Nucleic Acids Res. 1982, 10, 6223-6234.

${ }^{28}$ Geze, M.; Blanchard, P.; Fourrey, J. L.; Robert-Gero, M. Synthesis of sinefungin and its C-6' epimer. J. Am. Chem. Soc. 1983, 105, 76387640 .

${ }^{20}$ Buchanan, J. G.; Flinn, A.; Mundill, P. H. C.; Wightman, R. H. Approaches to the synthesis of sinefungin via nitroaldol reactions. $\mathrm{Nu}$ cleosides Nucleotides 1986, 5, 313-323.

${ }^{30}$ Barton, D. H. R.; Géro, S. D.; Quiclet-Sire, B.; Samadi, M. Expedient synthesis of natural $(S)$-sinefungin and of its C-6' epimer. $J$. Chem. Soc., Perkin Trans. 1 1991, 981-985.

${ }^{31}$ (a) Ghosh, A. K.; Liu, W. Total synthesis of (+)-sinefungin. J. Org. Chem. 1996, 61, 6175-6182. (b) Ghosh, A. K.; Wang, Y. Synthetic studies of nucleoside antibiotics: a formal synthesis of $(+)$-sinefungin. J. Chem. Soc., Perkin Trans. 1 1999, 3597-3601.

${ }^{32}$ Maguire, M. P.; Feldman, P. L.; Rapoport, H. Stereoselective synthesis and absolute stereochemistry of sinefungin. J. Org. Chem. 1990, 55, 948-955.

${ }^{33}$ Policarpo, R. L.; Decultot, L.; May, E.; Kuzmič, P.; Carlson, S.; Huang, D.; Chu, V.; Wright, B. A.; Dhakshinamoorthy, S.; Kannt, A.; Rani, S.; Dittakavi, S.; Panarese, J. D.; Gaudet, R.; Shair, M. D. Highaffinity alkynyl bisubstrate inhibitors of nicotinamide $N$-methyltransferase (NNMT). J. Med. Chem. 2019, 62, 9837-9873.

${ }^{34}$ Moreau, C.; Kirchberger, T.; Swarbrick, J. M.; Bartlett, S. J.; Fliegert, R.; Yorgan, T.; Bauche, A.; Harneit, A.; Guse, A. H.; Potter, B. V. Structure-activity relationship of adenosine 5 '-diphosphoribose at the transient receptor potential melastatin 2 (TRPM2) channel: rational design of antagonists. J. Med. Chem. 2013, 56, 10079-10102.

${ }^{35}$ Cannon, J. S.; Overman, L. E. Palladium(II)-catalyzed enantioselective reactions using COP catalysts. Acc. Chem. Res. 2016, 49, 2220 2231.

${ }^{36}$ Anderson, C. E.; Kirsch, S. F.; Overman, L. E.; Richards, C. J.; Watson, M. P. Preparation of the COP catalysts: $[(S)-\mathrm{COP}-\mathrm{OAc}]_{2}$, $[(S)$ COP-Cl $]_{2}$, and (S)-COP-hfacac. Org. Synth. 2007, 84, 148-155.

${ }^{37}$ Ning, X.-S.; Wang, M.-M.; Yao, C.-Z.; Chen, X.-M.; Kang, Y.-B. tert-Butyl nitrite: organic redox cocatalyst for aerobic aldehyde-selective Wacker-Tsuji oxidation. Org. Lett. 2016, 18, 2700-2703.

${ }^{38}$ (a) Burk, M. J.; Feaster, J. E.; Nugent, W. A.; Harlow, R. L. Preparation and use of $C_{2}$-symmetric bis(phospholanes): production of $\alpha$ amino acid derivatives via highly enantioselective hydrogenation reactions. J. Am. Chem. Soc. 1993, 115, 10125-10138. (b) Burk, M. J.; Gross, M. F.; Martinez, J. P. Asymmetric catalytic synthesis of $\beta$ branched amino acids via highly enantioselective hydrogenation reactions. J. Am. Chem. Soc. 1995, 117, 9375-9376. (c) Burk, M. J. Modular phospholane ligands in asymmetric catalysis. Acc. Chem. Res. 2000, $33,363-372$.
${ }^{39}$ Pilkington, C. J.; Zanotti-Gerosa, A. Expanding the family of phospholane-based ligands: 1,2-bis(2,5-diphenylphospholano)ethane. Org. Lett. 2003, 5, 1273-1275.

*o Sniady, A.; Bedore, M. W.; Jamison, T. F. One-flow, multistep synthesis of nucleosides by Bronsted acid-catalyzed glycosylation. Angew. Chem. Int. Ed. 2011, 50, 2155-2158.

${ }^{41}$ Babault, N.; Allali-Hassani, A.; Li, F.; Fan, J.; Yue, A.; Ju, K.; Liu, F.; Vedadi, M.; Liu, J.; Jin, J. Discovery of bisubstrate inhibitors of nicotinamide $N$-methyltransferase (NNMT). J. Med. Chem. 2018, 61, $1541-1551$ 


\section{Asymmetric Total Synthesis of C9'-epi-Sinefungin}

\section{Supporting Information: Experimental Protocols, Compound Characterization and NMR Spectra}

Ludovic Decultot ${ }^{\mathrm{a}, \dagger}$, Rocco L. Policarpo ${ }^{\mathrm{a}, \dagger}$, Brandon Wright $^{\mathrm{a}}$, Danny Huang ${ }^{\mathrm{a}}$, and Matthew D. Shair ${ }^{\mathrm{a}}$

${ }^{a}$ Department of Chemistry \&6 Chemical Biology, Harvard University, Cambridge MA 02138, USA.

${ }^{\dagger}$ These authors contributed equally to this work. 


\section{Table of Contents}

1 List of Abbreviations $\quad 3$

2 General Procedures $\quad 4$

3 Materials $\quad 4$

4 Instrumentation $\quad 4$

5 Synthetic Procedures \& Compound Characterization $\quad 6$

5.1 Trichloroacetimidate $8 \ldots \ldots \ldots \ldots \ldots \ldots \ldots$

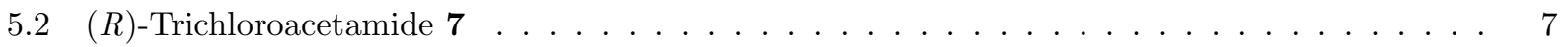

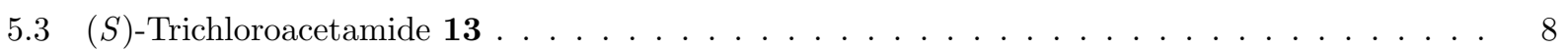

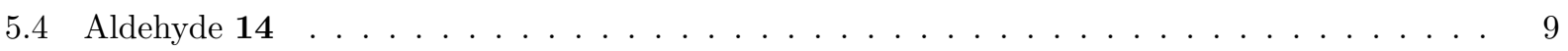

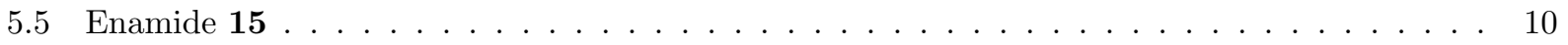

5.6 Protected Amino Acid $16 \ldots \ldots \ldots \ldots \ldots \ldots \ldots \ldots$

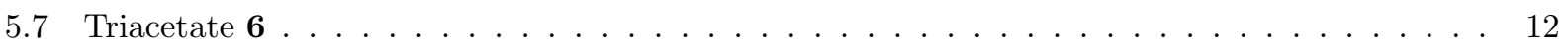

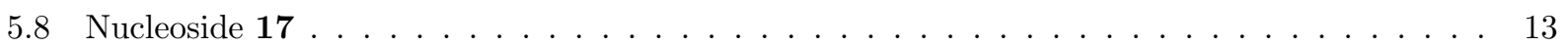

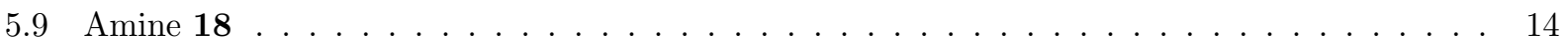

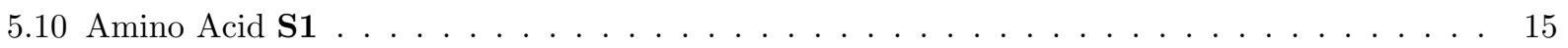

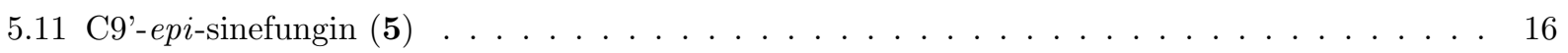

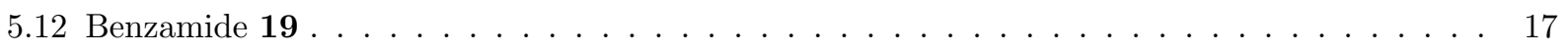

6 Analytical Characterization of C9'-epi-Sinefungin $r$

7 Small-Molecule X-Ray Crystallography 21

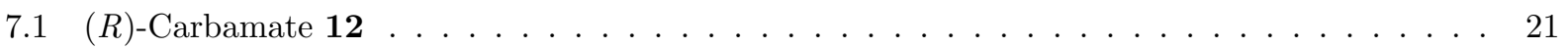

8 NMR Spectra $\quad 26$ 


\section{List of Abbreviations}

$\begin{array}{ll}\text { Ac } & \text { acetyl } \\ \text { BPE } & \text { bis(phospholano)ethane } \\ \text { BSA } & \text { N,O-bis(trimethylsilyl)acetamide } \\ \text { Cbz } & \text { benzyloxycarbonyl } \\ \text { COP } & \text { cobalt oxazoline palladacycle } \\ \text { DBU } & 1,8 \text {-diazabicyclo[5.4.0]undec-7-ene } \\ \text { DTBMP-OTf } & 2,6 \text {-di-tert-butyl-4-methylpyridinium triflate } \\ \text { equiv. } & \text { equivalent } \\ \text { M.S. } & \text { molecular sieves } \\ \text { MTBE } & \text { methyl tert-butyl ether } \\ \text { nbd } & \text { norbornadiene } \\ \text { Pyr } & \text { pyridine } \\ \text { rbf } & \text { round bottom flask } \\ \text { RT } & \text { room temperature } \\ \text { TCA } & \text { trichloroacetyl } \\ \text { TFA } & \text { trifluoroacetic acid } \\ \text { THF } & \text { tetrahydrofuran } \\ \text { TMG } & \end{array}$




\section{General Procedures}

Air and/or moisture sensitive reactions were performed under an atmosphere of nitrogen in a flame dried apparatus. Reactions were monitored by thin layer chromatography (TLC) using Merck $0.25 \mathrm{~mm}$ silica gel 60 covered glass backed plates $\mathrm{F}_{254}$. TLC plates were visualized under UV light and/or exposure to an acidic solution of $p$-anisaldehyde, an aqueous solution of ceric ammonium molybdate or an aqueous solution of potassium permanganate, followed by heating on a hot plate. Reactions were also monitored by analytical LCMS using an Agilent 6120 Quadrupole LC/MS and an Agilent 1260 Infinity II LC system equipped with (1) a Phenomenex Kinetex F5 column $(2.6 \mu \mathrm{m}, 100 \AA$ A, 100 x $3.0 \mathrm{~mm})$, (2) a Phenomenex Kinetex Biphenyl column $(2.6 \mu \mathrm{m}, 100 \AA \AA$, $100 \times 3.0 \mathrm{~mm})$ or $(3)$ a Thermo Scientific Accucore aQ C18 column $(2.6 \mu \mathrm{m}, 100 \times$ $2.1 \mathrm{~mm}$ ). Two methods were used: method $A$ for columns 1, 2, 3: solvent A: $0.1 \%(\mathrm{v} / \mathrm{v})$ formic acid in water, solvent B: $0.1 \%(\mathrm{v} / \mathrm{v})$ formic acid in $\mathrm{CH}_{3} \mathrm{CN}$, gradient elution: $5 \% \mathrm{~B}$ for 1 min then $5 \% \rightarrow 95 \%$ B over 8 min followed by $95 \% \mathrm{~B}$ for $2 \mathrm{~min}$ and reequilibration for $2 \mathrm{~min}$, flow rate: $0.55 \mathrm{~mL} / \mathrm{min}(1), 0.95 \mathrm{~mL} / \mathrm{min}$ (2), $0.70 \mathrm{~mL} / \mathrm{min}(3)$, column temperature: $50{ }^{\circ} \mathrm{C}$, UV detection: $254 \mathrm{~nm}$, and method $B$ for columns 2,3 : solvent A: $0.1 \%(\mathrm{v} / \mathrm{v})$ formic acid in water, solvent B: $0.1 \%(\mathrm{v} / \mathrm{v})$ formic acid in $\mathrm{CH}_{3} \mathrm{CN}$, gradient elution: $0 \%$ B for $1 \mathrm{~min}, 0 \% \rightarrow 40 \%$ B over $8 \mathrm{~min}, 40 \% \rightarrow 95 \%$ B over 2 min followed by $95 \%$ B for 2 min and reequilibration for $2 \mathrm{~min}$, flow rate: $0.95 \mathrm{~mL} / \mathrm{min}(2), 0.70 \mathrm{~mL} / \mathrm{min}(3)$, column temperature: $50{ }^{\circ} \mathrm{C}$, UV detection: $254 \mathrm{~nm}$. Flash column chromatography was performed using an Interchim PuriFlash 215 system with prepacked silica gel cartridges (Büchi FlashPure (35 to $45 \mu \mathrm{m}$ ), Büchi Reveleris HP (16 to $24 \mu \mathrm{m}$ ), Teledyne Isco RediSep Rf (35 to $70 \mu \mathrm{m}$ ) and Teledyne Isco RediSep Rf Gold (20 to $40 \mu \mathrm{m}$ )). Preparative HPLC was performed at room temperature on an Agilent 1260 Infinity II Preparative LC system equipped with a Kromasil C18 column $(10 \mu \mathrm{m}, 100 \AA, 21.2 \times 250 \mathrm{~mm})$ using solvents A $(0.1 \%$ (v/v) TFA in water) and $\mathrm{B}(0.1 \%(\mathrm{v} / \mathrm{v})$ TFA in acetonitrile) at flow rate $10 \mathrm{~mL} / \mathrm{min}$ delivering gradients specified in individual experimental protocols and with UV detection at $254 \mathrm{~nm}$.

\section{Materials}

Commercial reagents and solvents were used as received unless specified otherwise. The molarity of $n$ butyllithium solutions was determined by titration using $N$-benzylbenzamide (average of three determinations). ${ }^{1}$

\section{Instrumentation}

${ }^{1} \mathrm{H}$ NMR spectra were recorded on Varian INOVA-600 or Varian INOVA-500 spectrometers at ambient temperature. Chemical shifts are reported in ppm ( $\delta$ scale $)$ relative to residual undeuterated solvent $\left(\mathrm{CDCl}_{3}\right.$ : $\left.7.26\left(\mathrm{CHCl}_{3}\right), \mathrm{CD}_{3} \mathrm{CN}: 1.94\left(\mathrm{CD}_{2} \mathrm{HCN}\right), \mathrm{D}_{2} \mathrm{O}: 4.79(\mathrm{HOD})\right)$. Data are reported as follows: chemical shift $(\delta \mathrm{ppm})$ (integration, multiplicity $(\mathrm{s}=$ singlet, $\mathrm{d}=$ doublet, $\mathrm{t}=$ triplet, $\mathrm{q}=$ quartet, $\mathrm{m}=$ multiplet, $\mathrm{br}$ = broad, app = apparent, or a combination of these), coupling constant(s) $J(\mathrm{~Hz}))$. When a mixture of deuterated solvents is employed, the residual undeuterated solvent used for reference is indicated in bold. ${ }^{13} \mathrm{C}$ NMR spectra were recorded on Varian INOVA-500, Varian MERCURY-400 and JEOL J400 spectrometers at ambient temperature. Data are reported as follows: chemical shifts ( $\delta$ scale, ppm) relative to the carbon resonances of the solvent $\left(\mathrm{CDCl}_{3}: 77.16, \mathrm{CD}_{3} \mathrm{CN}: 1.32\right)$. When a mixture of deuterated solvents is employed,

1 Burchat, A. F.; Chong, J.; Nielsen, N. J. Organomet. Chem. 1997, 542, 281-283. 
the solvent used for reference is indicated in bold. Infrared (FTIR) spectra were recorded on a Bruker Alpha FT-IR spectrophotometer. Data is reported in frequency of absorption $\left(\mathrm{cm}^{-1}\right)$. The IR spectrum of each compound (solid or liquid) was acquired directly on a thin layer at ambient temperature. High resolution mass spectra (HRMS) were recorded on a Bruker micrOTOF-Q II or on an Agilent 6220 LC-TOF equipped with an electrospray ionization (ESI+). The structure of carbamate $\mathbf{1 2}$ was obtained with the assistance of Dr. Shao-Liang Zheng at the X-ray diffraction facility of the Department of Chemistry \& Chemical Biology at Harvard University. The analytical characterization of C9'-epi-sinefungin was obtained with the assistance of Jennifer X. Wang at the small molecule mass spectrometry facility at Harvard University. 


\section{Synthetic Procedures \& Compound Characterization}

\subsection{Trichloroacetimidate 8}

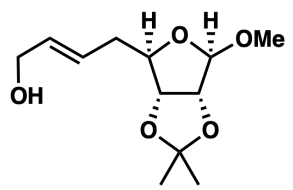

11

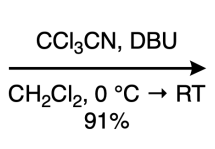

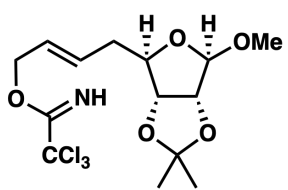

To a solution of known allylic alcohol $\mathbf{1 1}^{2}(7.08 \mathrm{~g}, 29.9 \mathrm{mmol})$ in $\mathrm{CH}_{2} \mathrm{Cl}_{2}(200 \mathrm{~mL})$ at $0{ }^{\circ} \mathrm{C}$, were added DBU (0.87 mL, $5.8 \mathrm{mmol}, 20 \mathrm{~mol} \%$ ) and trichloroacetonitrile ( $4.31 \mathrm{~mL}, 43.5 \mathrm{mmol}, 1.5$ equiv.) sequentially. The resulting mixture was allowed to warm up to RT over 30 min and was stirred at RT for an additional $1.5 \mathrm{~h}$. Volatiles were removed in vacuo. Crude material was purified by silica gel chromatography (hexanes/EtOAc, $0 \rightarrow 30 \%)$ to afford trichloroacetimidate $8(10.3 \mathrm{~g}, 26.5 \mathrm{mmol}, 91 \%)$ as a colorless oil.

$\mathrm{R}_{\mathrm{f}}=0.34$ (hexanes/EtOAc, 80:20);

FTIR (thin film), $\mathrm{cm}^{-1}: \nu_{\max } 3343,2989,2937,1662,1442,1381,1373,1286,1209,1088,1058$;

${ }^{1} \mathrm{H}$ NMR $\left(600 \mathrm{MHz}, \mathrm{CDCl}_{3}\right): \delta 8.29(\mathrm{~s}, 1 \mathrm{H}), 5.91-5.85(\mathrm{~m}, 1 \mathrm{H}), 5.83-5.76(\mathrm{~m}, 1 \mathrm{H}), 4.95(\mathrm{~s}, 1 \mathrm{H}), 4.81-4.74$ $(\mathrm{m}, 2 \mathrm{H}), 4.61(\mathrm{~d}, J=5.9 \mathrm{~Hz}, 1 \mathrm{H}), 4.56(\mathrm{dd}, J=5.9,1.1 \mathrm{~Hz}, 1 \mathrm{H}), 4.25(\operatorname{app} \mathrm{td}, J=7.8,1.1 \mathrm{~Hz}, 1 \mathrm{H}), 3.33$ (s, 3H), 2.45 (dddd, $J=14.4,7.8,6.4,1.3 \mathrm{~Hz}, 1 \mathrm{H}), 2.37-2.30(\mathrm{~m}, 1 \mathrm{H}), 1.47(\mathrm{~s}, 3 \mathrm{H}), 1.31$ (s, 3H);

${ }^{13} \mathrm{C} \mathrm{NMR}\left(126 \mathrm{MHz}, \mathrm{CDCl}_{3}\right): \delta 162.4,132.1,126.2,112.3,109.6,91.5,86.2,85.5,83.5,69.5,55.0,38.1,26.5$, 25.1

HRMS (ESI+): calcd. for $\left[\mathrm{C}_{14} \mathrm{H}_{20} \mathrm{Cl}_{3} \mathrm{~N}_{1} \mathrm{O}_{5} \mathrm{Na}\right]^{+} 410.0299$, meas. 410.0292, $\Delta 1.7 \mathrm{ppm}$.

${ }^{2}$ K. Ghosh, A.; Wang, Y. J. Chem. Soc., Perkin Trans. 1 1999, 3597-3601. 


\section{$5.2 \quad(R)$-Trichloroacetamide 7}

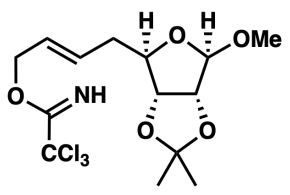

8

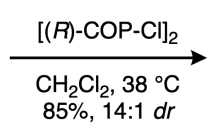

$85 \%, 14: 1 d r$

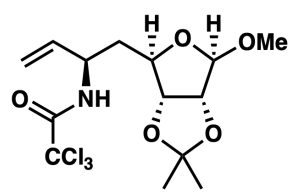

7

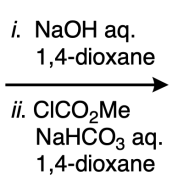

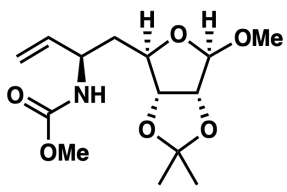

12

To a vial charged with trichloroacetimidate $8(1.22 \mathrm{~g}, 3.14 \mathrm{mmol})$ and $[(R)-\mathrm{COP}-\mathrm{Cl}]_{2}{ }^{3}(115 \mathrm{mg}, 78 \mathrm{\mu mol}$, $2.5 \mathrm{~mol} \%$ ) was added $\mathrm{CH}_{2} \mathrm{Cl}_{2}(9.2 \mathrm{~mL})$. The vial headspace was purged with argon and the vessel was sealed. The resulting mixture was covered with foil and was stirred at $38^{\circ} \mathrm{C}$ for $50 \mathrm{~h}$. Volatiles were removed in vacuo. ${ }^{1} \mathrm{H}$ NMR analysis of the crude mixture revealed a 14:1 $d r$. Crude material was purified by silica gel chromatography (hexanes/MTBE, $0 \rightarrow 45 \%$ ) to afford $(R)$-trichloroacetamide 7 (1.04 g, 2.68 mmol, 85\%) as a single diastereomer, as a colorless oil. Trichloroacetamide $\mathbf{7}$ could be converted to carbamate 12 ( $i$. 1,4-dioxane / $1 \mathrm{M} \mathrm{NaOH}$ aq. (1:1), rt; ii. $\mathrm{ClCO}_{2} \mathrm{Me}$ (1.2 equiv.), 1,4-dioxane / sat. $\mathrm{NaHCO}_{3}$ aq. (1:1), 0 ${ }^{\circ} \mathrm{C}$ ) which crystallized when stored as a neat oil at $-20{ }^{\circ} \mathrm{C}$ overnight. A small molecule X-ray structure was obtained, confirming its absolute configuration as shown in subsection 7.1.

$\mathrm{R}_{\mathrm{f}}=0.27$ (hexanes/EtOAc, 80:20);

FTIR (thin film) $\mathrm{cm}^{-1}: \nu_{\max } 3298,2980,2938,1711,1691,1531,1381,1372,1274,1260,1236,1209,1191$, 1160, 1105, 1091, 1058;

${ }^{1} \mathrm{H} \mathrm{NMR}\left(500 \mathrm{MHz}_{\mathrm{CDCl}}\right): \delta 7.65(\mathrm{br} \mathrm{d}, J=7.9 \mathrm{~Hz}, 1 \mathrm{H}), 5.83(\mathrm{ddd}, J=17.3,10.3,5.0 \mathrm{~Hz}, 1 \mathrm{H}), 5.29-5.25$ $(\mathrm{m}, 2 \mathrm{H}), 5.00(\mathrm{~s}, 1 \mathrm{H}), 4.70-4.63(\mathrm{~m}, 1 \mathrm{H}), 4.61(\mathrm{~d}, J=6.0 \mathrm{~Hz}, 1 \mathrm{H}), 4.56(\mathrm{ddd}, J=6.0,1.6,0.5 \mathrm{~Hz}, 1 \mathrm{H})$, 4.36 (ddd, $J=11.4,3.2,1.6 \mathrm{~Hz}, 1 \mathrm{H}), 3.40(\mathrm{~s}, 3 \mathrm{H}), 2.05$ (ddd, $J=14.4,11.4,4.5 \mathrm{~Hz}, 1 \mathrm{H}), 1.87$ (dddd, $J=$ $14.4,5.6,3.2,0.5 \mathrm{~Hz}, 1 \mathrm{H}), 1.48(\mathrm{~s}, 3 \mathrm{H}), 1.32(\mathrm{~s}, 3 \mathrm{H})$;

${ }^{13} \mathrm{C} \mathrm{NMR}\left(126 \mathrm{MHz}, \mathrm{CDCl}_{3}\right): \delta 161.4,135.4,116.5,112.8,110.3,92.8,84.9,84.5,83.9,55.8,51.6,37.9,26.6$, 25.0

HRMS (ESI+): calcd. for $\left[\mathrm{C}_{14} \mathrm{H}_{20} \mathrm{Cl}_{3} \mathrm{~N}_{1} \mathrm{O}_{5} \mathrm{Na}\right]^{+}$410.0299, meas. 410.0299, $\Delta 0.2 \mathrm{ppm}$.

$3[(R)-\mathrm{COP}-\mathrm{Cl}]_{2}$ (CAS: 612065-00-6) was prepared from $[(R) \text {-COP-OAc }]_{2}$ (CAS: 849592-74-1), commercially available from Millipore Sigma (catalog \#: 661708), following the protocol outlined in Org. Synth. 2007, 84, 148-155. 


\section{$5.3 \quad(S)$-Trichloroacetamide 13}

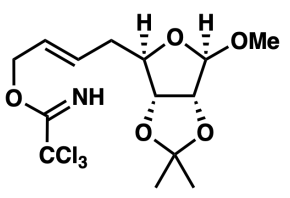

8

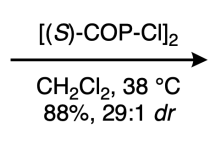

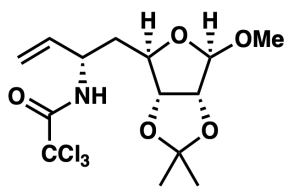

13

To a vial charged with trichloroacetimidate $8(101 \mathrm{mg}, 0.260 \mathrm{mmol})$ and $[(S) \text {-COP-Cl }]_{2}{ }^{4}(19 \mathrm{mg}, 13$ pmol, $5 \mathrm{~mol} \%)$ was added $\mathrm{CH}_{2} \mathrm{Cl}_{2}(0.75 \mathrm{~mL})$. The vial headspace was purged with argon and the vessel was sealed. The resulting mixture was covered with foil and was stirred at $38{ }^{\circ} \mathrm{C}$ for $48 \mathrm{~h}$. Volatiles were removed in vacuo. ${ }^{1} \mathrm{H}$ NMR analysis of the crude mixture revealed a 29:1 $d r$. Crude material was purified by silica gel chromatography (hexanes/MTBE, $0 \rightarrow 45 \%$ ) to afford $(S)$-trichloroacetamide $\mathbf{1 3}(89 \mathrm{mg}, 0.23$ mmol, $88 \%$ ) as a single diastereomer, as a colorless oil.

$\mathrm{R}_{\mathrm{f}}=0.27$ (hexanes/EtOAc, 80:20);

FTIR (thin film), cm ${ }^{-1}: \nu_{\max } 3335,2989,2938,1698,1517,1382,1210,1107,1093,1062$;

${ }^{1} \mathrm{H}$ NMR $\left(500 \mathrm{MHz}, \mathrm{CDCl}_{3}\right): \delta 6.97(\mathrm{br} \mathrm{d}, J=7.4 \mathrm{~Hz}, 1 \mathrm{H}), 5.81(\mathrm{ddd}, J=17.2,10.4,6.5 \mathrm{~Hz}, 1 \mathrm{H}), 5.34$ (app dt, $J=17.2,1.0 \mathrm{~Hz}, 1 \mathrm{H}), 5.27$ (app dt, $J=10.4,1.0 \mathrm{~Hz}, 1 \mathrm{H}), 4.98(\mathrm{~s}, 1 \mathrm{H}), 4.62(\mathrm{~d}, J=5.9 \mathrm{~Hz}, 1 \mathrm{H})$, $4.59-4.51(\mathrm{~m}, 2 \mathrm{H}), 4.25$ (ddd, $J=10.7,4.2,1.3 \mathrm{~Hz}, 1 \mathrm{H}), 3.43(\mathrm{~s}, 3 \mathrm{H}), 1.99$ (ddd, $J=13.8,10.7,6.8 \mathrm{~Hz}$, $1 \mathrm{H}), 1.83(\mathrm{ddd}, J=13.8,8.1,4.2 \mathrm{~Hz}, 1 \mathrm{H}), 1.48(\mathrm{~s}, 3 \mathrm{H}), 1.31(\mathrm{~s}, 3 \mathrm{H})$;

${ }^{13} \mathrm{C} \mathrm{NMR}\left(126 \mathrm{MHz}, \mathrm{CDCl}_{3}\right): \delta 161.1,135.5,117.6,112.7,110.1,92.8,85.3,84.5,84.3,55.7,52.4,39.4,26.6$, 25.1

HRMS (ESI+): calcd. for $\left[\mathrm{C}_{14} \mathrm{H}_{20} \mathrm{Cl}_{3} \mathrm{~N}_{1} \mathrm{O}_{5} \mathrm{Na}\right]^{+} 410.0299$, meas. 410.0304, $\Delta 1.2 \mathrm{ppm}$.

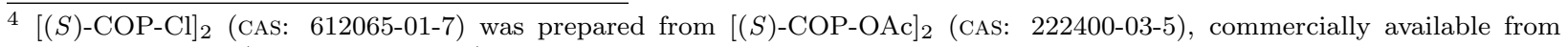
Millipore Sigma (catalog \#: 661716), following the protocol outlined in Org. Synth. 2007, 84, 148-155. 


\subsection{Aldehyde 14}

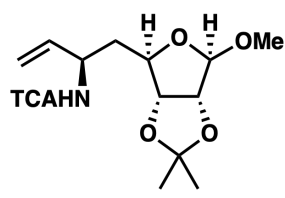

7

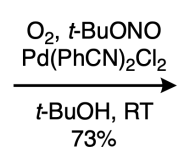

$73 \%$

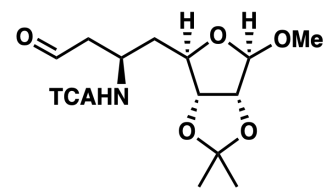

14

To an oversized flask (250 mL rbf, in order to increase the surface area of exposed solvent) charged with $\mathrm{Pd}(\mathrm{PhCN})_{2} \mathrm{Cl}_{2}(99 \mathrm{mg}, 0.257 \mathrm{mmol}, 10 \mathrm{~mol} \%$ ), $t$-BuOH $(36 \mathrm{~mL})$ and $t$-BuONO (122 $\mu \mathrm{L}, 1.03 \mathrm{mmol}, 40 \mathrm{~mol}$ \%) was added a solution of alkene $7(1.00 \mathrm{~g}, 2.57 \mathrm{mmol})$ in $t$-BuOH $(4 \mathrm{~mL})$. The flask headspace was purged with $\mathrm{O}_{2}$ and the reaction mixture was stirred at RT under an atmosphere of $\mathrm{O}_{2}$ (balloon) for 40 h. Volatiles were removed in vacuo. Crude material was purified by silica gel chromatography (hexane/EtOAc, $0 \rightarrow$ $100 \%$ ) to yield aldehyde 14 (760 mg, $1.88 \mathrm{mmol}, 73 \%$ ) as a colorless oil, which was best used immediately in the subsequent reaction. ${ }^{5}$

$\mathrm{R}_{\mathrm{f}}=0.31$ (cyclohexane/EtOAc, 50:50);

FTIR (thin film), $\mathrm{cm}^{-1}: \nu_{\max } 3325,2988,2937,2835,2734,1694,1518,1382,1373,1210,1160,1092,1059$;

${ }^{1} \mathrm{H} \mathrm{NMR}\left(500 \mathrm{MHz}, \mathrm{CDCl}_{3}\right): \delta 9.79(\mathrm{~s}, 1 \mathrm{H}), 7.60(\mathrm{~d}, J=8.2 \mathrm{~Hz}, 1 \mathrm{H}), 4.97(\mathrm{~s}, 1 \mathrm{H}), 4.60(\mathrm{~d}, J=5.9 \mathrm{~Hz}$, $1 \mathrm{H}), 4.53(\mathrm{dd}, J=6.0,1.2 \mathrm{~Hz}, 1 \mathrm{H}), 4.45$ (ddt, $J=13.4,8.1,5.3 \mathrm{~Hz}, 1 \mathrm{H}), 4.27$ (ddd, $J=11.5,3.4,1.2 \mathrm{~Hz}$, $1 \mathrm{H}), 3.39(\mathrm{~s}, 3 \mathrm{H}), 3.00(\mathrm{ddd}, J=18.3,5.2,1.3 \mathrm{~Hz}, 1 \mathrm{H}), 2.85(\mathrm{dd}, J=18.3,6.1 \mathrm{~Hz}, 1 \mathrm{H}), 2.08(\mathrm{ddd}, J=$ 14.5, 8.1, 3.4 Hz, 1H), 1.89 (ddd, $J=14.5,11.6,4.4 \mathrm{~Hz}, 1 \mathrm{H}), 1.47$ (s, 3H), $1.31(\mathrm{~s}, 3 \mathrm{H})$;

${ }^{13} \mathrm{C} \mathrm{NMR}\left(126 \mathrm{MHz}, \mathrm{CDCl}_{3}\right): \delta 200.1,161.7,112.9,110.5,85.1,84.5,83.9,55.8,46.7,45.5,37.7,28.9,26.6$, 25.1

HRMS (ESI+): calcd. for $\left[\mathrm{C}_{14} \mathrm{H}_{20} \mathrm{Cl}_{3} \mathrm{~N}_{1} \mathrm{O}_{6} \mathrm{Na}\right]^{+}$426.0248, meas. 426.0240, $\Delta 2.0$ ppm.

\footnotetext{
5 While in this protocol aldehyde $\mathbf{1 4}$ was purified by silica gel chromatography, it was found that subjection of the crude residue to the Horner-Wadsworth-Emmons olefination conditions reliably generated enamide $\mathbf{1 5}$ in $74 \%$ yield over two steps (oxidation/olefination) from $0.7 \mathrm{mmol}$ of alkene 7 .
} 


\subsection{Enamide 15}

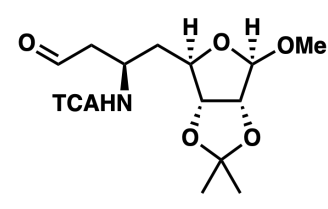

14
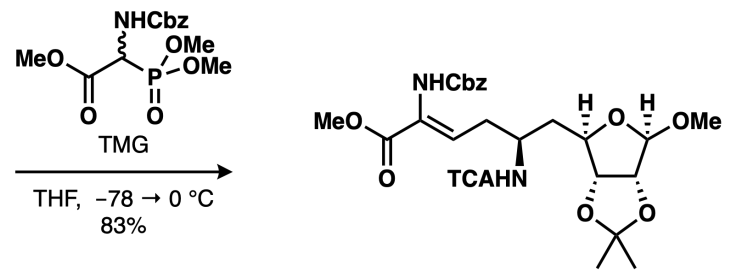

15

To a stirred suspension of ( \pm -Cbz- $\alpha$-phosphonoglycine trimethyl ester ${ }^{6}$ (747 mg, 2.25 mmol, 1.2 equiv.) in THF (10.0 mL) at $-78^{\circ} \mathrm{C}$, was added 1,1,3,3-tetramethylguanidine ( $0.26 \mathrm{~mL}, 2.1 \mathrm{mmol}, 1.1$ equiv.). The resulting mixture was stirred at $-78^{\circ} \mathrm{C}$ for $10 \mathrm{~min}$, before the slow addition of a solution of aldehyde 14 (760 $\mathrm{mg}, 1.88 \mathrm{mmol})$ in THF $(5.0 \mathrm{~mL})$ over $3 \mathrm{~min}$. The reaction mixture was stirred at $-78{ }^{\circ} \mathrm{C}$ for $30 \mathrm{~min}$ and then placed in an ice/water bath and stirred for $10 \mathrm{~min}$. The reaction was quenched by the addition of a $10 \%$ AcOH aq. solution $(5 \mathrm{~mL})$ and water $(20 \mathrm{~mL})$ was added. The layers were separated and the aqueous phase was extracted with $\mathrm{CH}_{2} \mathrm{Cl}_{2}(3 \times 40 \mathrm{~mL})$. The combined organic extracts were washed with water $(25 \mathrm{~mL})$ and brine $(25 \mathrm{~mL})$, passed through a phase separator (Biotage Isolute) and concentrated. Crude material was purified by silica gel chromatography (2,2,4-trimethylpentane/EtOAc, $0 \rightarrow 75 \%)$ to afford $Z$ enamide 15 (945 mg, $1.55 \mathrm{mmol}, 83 \%$ ) as a clear viscous oil.

$\mathrm{R}_{\mathrm{f}}=0.42$ (hexanes/EtOAc, 50:50);

FTIR (thin film), $\mathrm{cm}^{-1}: \nu_{\max } 3315,2952,1699,1514,1438,1382,1374,1234,1159,1089,1055$;

${ }^{1} \mathrm{H} \mathrm{NMR}\left(500 \mathrm{MHz}, \mathrm{CDCl}_{3}\right): \delta 8.05$ (br s, 1H), 7.41-7.31 (m, 5H), 6.53 (br s, 1H), 6.52 (dd, J=10.0, 5.8 $\mathrm{Hz}, 1 \mathrm{H}), 5.14(\mathrm{~s}, 2 \mathrm{H}), 4.97(\mathrm{~s}, 1 \mathrm{H}), 4.61(\mathrm{~s}, 2 \mathrm{H}), 4.28(\mathrm{dd}, J=7.6,7.1 \mathrm{~Hz}, 1 \mathrm{H}), 4.26-4.19(\mathrm{~m}, 1 \mathrm{H}), 3.76(\mathrm{~s}$, $3 \mathrm{H}), 3.36(\mathrm{~s}, 3 \mathrm{H}), 2.65(\mathrm{dt}, J=15.0,9.9 \mathrm{~Hz}, 1 \mathrm{H}), 2.54(\mathrm{dt}, J=15.0,5.9 \mathrm{~Hz}, 1 \mathrm{H}), 1.86(\mathrm{dd}, J=8.1,6.0$ $\mathrm{Hz}, 2 \mathrm{H}), 1.47(\mathrm{~s}, 3 \mathrm{H}), 1.31(\mathrm{~s}, 3 \mathrm{H})$;

${ }^{13} \mathrm{C} \mathrm{NMR}\left(151 \mathrm{MHz}, \mathrm{CDCl}_{3}\right): \delta 164.6,162.1,154.2,135.7,130.9,128.7,128.5,128.4,112.6,110.1,92.8,85.3$, $84.4,83.9,67.8,55.4,52.8,48.7,39.9,33.0,26.5,25.0$;

HRMS (ESI+): calcd. for $\left[\mathrm{C}_{25} \mathrm{H}_{31} \mathrm{Cl}_{3} \mathrm{~N}_{2} \mathrm{O}_{9} \mathrm{Na}\right]^{+}$631.0987, meas. 631.0997, $\Delta 1.6 \mathrm{ppm}$.

$\overline{6}$ ( \pm )-Cbz- $a$-phosphonoglycine trimethyl ester, CAS: 88568-95-0, was purchased from Chem-Impex International, Inc. (catalog \#: 14125). 


\subsection{Protected Amino Acid 16}

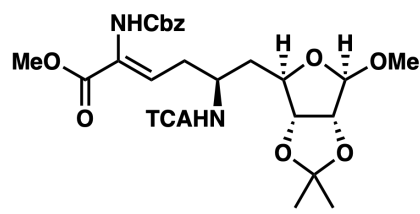

15

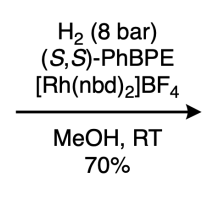

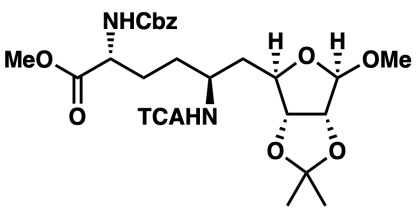

16

In a glove box, $\left[\mathrm{Rh}(\mathrm{nbd})_{2}\right] \mathrm{BF}_{4}{ }^{7}(24 \mathrm{mg}, 0.063 \mathrm{mmol}, 19 \mathrm{~mol} \%)$ and $(S, S)-\mathrm{PhBPE}^{8}$ (34 mg, $0.067 \mathrm{mmol}$, $20 \mathrm{~mol} \%$ ) were added to a vial containing a stir bar. The vial was capped with a septum, removed from the glovebox and placed under an atmosphere of nitrogen. $\mathrm{MeOH}(4 \mathrm{~mL})$ was added and the red/orange solution was stirred for 15 min before being transferred to a pressure tube ${ }^{9}$ equipped with a side arm through which hydrogen was flowing freely. A solution of enamide 15 (203 $\mathrm{mg}, 0.333 \mathrm{mmol})$ in $\mathrm{MeOH}(4 \mathrm{~mL})$ was added through the side arm and the side arm was sealed. The vessel was pressurized to 4 bar of hydrogen and the pressure was released to purge the vessel $(3 \times)$. After the third purge, a hydrogen pressure of 8 bar was applied and the reaction mixture was stirred vigorously at RT for $15 \mathrm{~min}$, before releasing

the pressure. Volatiles were removed in vacuo. Crude material was purified by silica gel chromatography (2,2,4-trimethylpentane/EtOAc, $0 \rightarrow 70 \%)$ to yield protected amino acid 16 (143 mg, $0.234 \mathrm{mmol}, 70 \%)$ as an amorphous white solid. ${ }^{1} \mathrm{H}$ NMR analysis indicated the presence of only one diastereomer.

$\mathrm{R}_{\mathrm{f}}=0.39$ (cyclohexane/EtOAc, 50:50);

FTIR (thin film), $\mathrm{cm}^{-1}: \nu_{\max } 3325,2937,1694,1522,1454,1382,1238,1211,1161,1090,1057$;

${ }^{1} \mathrm{H}$ NMR $\left(600 \mathrm{MHz}, \mathrm{CDCl}_{3}\right): \delta 7.38-7.28(\mathrm{~m}, 5 \mathrm{H}), 7.25(\mathrm{br} \mathrm{d}, J=8.4 \mathrm{~Hz}, 1 \mathrm{H}), 5.49(\mathrm{br} \mathrm{d}, J=8.2 \mathrm{~Hz}, 1 \mathrm{H})$, $5.09(\mathrm{~s}, 2 \mathrm{H}), 4.95(\mathrm{~s}, 1 \mathrm{H}), 4.58(\mathrm{~d}, J=5.9 \mathrm{~Hz}, 1 \mathrm{H}), 4.52(\mathrm{dd}, J=5.9,1.3 \mathrm{~Hz}, 1 \mathrm{H}), 4.43-4.35(\mathrm{~m}, 1 \mathrm{H}), 4.26$ (ddd, $J=11.2,3.9,1.3 \mathrm{~Hz}, 1 \mathrm{H}), 4.11-4.04(\mathrm{~m}, 1 \mathrm{H}), 3.72(\mathrm{~s}, 3 \mathrm{H}), 3.34(\mathrm{~s}, 3 \mathrm{H}), 1.98-1.89(\mathrm{~m}, 1 \mathrm{H}), 1.84$ (ddd, $J=15.0,10.9,3.9 \mathrm{~Hz}, 1 \mathrm{H}), 1.80-1.67(\mathrm{~m}, 3 \mathrm{H}), 1.67-1.59(\mathrm{~m}, 1 \mathrm{H}), 1.46(\mathrm{~s}, 3 \mathrm{H}), 1.29(\mathrm{~s}, 3 \mathrm{H})$;

${ }^{13} \mathrm{C} \mathrm{NMR}\left(151 \mathrm{MHz}, \mathrm{CDCl}_{3}\right): \delta 172.5,161.8,156.1,136.2,128.6,128.3,128.2,112.8,110.2,92.9,85.1,84.5$, 83.6, 67.2, 55.7, 53.8, 52.7, 49.6, 38.6, 30.0, 29.4, 26.6, 25.1;

HRMS (ESI+): calcd. for $\left[\mathrm{C}_{25} \mathrm{H}_{34} \mathrm{Cl}_{3} \mathrm{~N}_{2} \mathrm{O}_{9}\right]^{+}$611.1324, meas. 611.1323, $\Delta 0.3 \mathrm{ppm}$.

$7\left[\mathrm{Rh}(\mathrm{nbd})_{2}\right] \mathrm{BF}_{4}, \mathrm{CAS}: 36620-11-8$, was obtained from Strem Chemicals, Inc. (catalog : 45-0230).

8 (S,S)-PhBPE ligand, CAS: 824395-67-7, was obtained from Strem Chemicals, Inc. (catalog : 15-0474).

9 The pressure vessel, $60 \mathrm{~mL}$, was obtained from Ace Glass (catalog : 8649-115). 


\subsection{Triacetate 6}

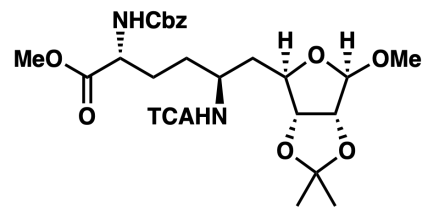

16

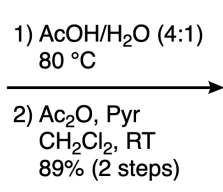

89\% (2 steps)

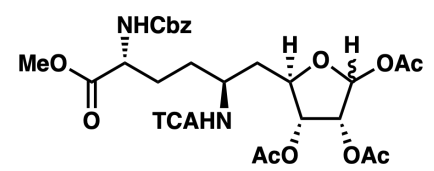

6

To a vial charged with acetonide $16(145 \mathrm{mg}, 0.237 \mathrm{mmol})$ was added a 4:1 mixture of acetic acid and water $(10 \mathrm{~mL})$ at RT. The resulting mixture was stirred at $80^{\circ} \mathrm{C}$ for $18 \mathrm{~h}$. Volatiles were removed in vacuo and the residue was dried by azeotropic distillation with benzene $(2 \times 1.5 \mathrm{~mL})$. Crude material was used directly in the next step without further purification.

To a vial charged with the crude triol was added a mixture of pyridine $(1.53 \mathrm{~mL}, 19.0 \mathrm{mmol}, 80$ equiv.) and $\mathrm{Ac}_{2} \mathrm{O}$ (0.90 mL, $9.5 \mathrm{mmol}, 40$ equiv.). The resulting mixture was stirred at $\mathrm{RT}$ for $1 \mathrm{~h}$ and the volatiles were removed in vacuo. Crude material was purified by silica gel chromatography (2,2,4-trimethylpentane/EtOAc, $2 \rightarrow 70 \%)$ to afford triacetate 6 (145 mg, $0.212 \mathrm{mmol}, 89 \%$ over 2 steps) as a 2:1 mixture of diastereomers, as a white amorphous solid.

$\mathrm{R}_{\mathrm{f}}=0.36 / 0.45$ (hexanes/EtOAc, 40:60);

FTIR (thin film), $\mathrm{cm}^{-1}: \nu_{\max } 3336,2955,1743,1703,1522,1454,1436,1373,1217,1112,1047,1011$;

${ }^{1} \mathrm{H} \mathrm{NMR}\left(600 \mathrm{MHz}, \mathrm{CDCl}_{3}\right): \delta 7.47(\mathrm{br} \mathrm{d}, J=8.8 \mathrm{~Hz}, 3 \mathrm{H}), 7.39-7.29(\mathrm{~m}, 15 \mathrm{H}), 6.37(\mathrm{~d}, J=4.6 \mathrm{~Hz}, 1 \mathrm{H})$, $6.18(\mathrm{~s}, 2 \mathrm{H}), 5.49-5.41(\mathrm{~m}, 3 \mathrm{H}), 5.29(\mathrm{dd}, J=4.7,0.8 \mathrm{~Hz}, 2 \mathrm{H}), 5.19(\mathrm{dd}, J=6.9,4.6 \mathrm{~Hz}, 1 \mathrm{H}), 5.12(\mathrm{dd}, J$ $=7.6,4.7 \mathrm{~Hz}, 2 \mathrm{H}), 5.11-5.09(\mathrm{~m}, 6 \mathrm{H}), 4.95(\mathrm{dd}, J=6.9,4.0 \mathrm{~Hz}, 1 \mathrm{H}), 4.40-4.31(\mathrm{~m}, 6 \mathrm{H}), 4.17-4.11(\mathrm{~m}, 2 \mathrm{H})$, 4.11-4.06 (m, 1H), $3.73(\mathrm{~s}, 6 \mathrm{H}), 3.73(\mathrm{~s}, 3 \mathrm{H}), 2.13(\mathrm{~s}, 6 \mathrm{H}), 2.10(\mathrm{~s}, 3 \mathrm{H}), 2.09(\mathrm{~s}, 3 \mathrm{H}), 2.07(\mathrm{~s}, 3 \mathrm{H}), 2.06(\mathrm{~s}, 6 \mathrm{H})$, $2.05(\mathrm{~s}, 6 \mathrm{H}), 1.98-1.90(\mathrm{~m}, 3 \mathrm{H}), 1.82(\mathrm{ddd}, J=14.9,11.4,3.6 \mathrm{~Hz}, 3 \mathrm{H}), 1.79-1.67(\mathrm{~m}, 9 \mathrm{H}), 1.66-1.60(\mathrm{~m}, 3 \mathrm{H})$;

${ }^{13} \mathrm{C}$ NMR $\left(151 \mathrm{MHz}, \mathrm{CDCl}_{3}\right): \delta 172.4,170.3,169.8,169.5,169.4,169.4,168.8,161.8,161.7,156.1,156.1$, 136.2, 128.6, 128.6, 128.3, 128.2, 128.1, 98.0, 93.7, 92.8, 92.6, 80.5, 78.1, 77.2, 74.0, 73.7, 72.8, 69.6, 67.1, $53.8,52.6,49.5,49.3,37.7,36.8,29.6,29.5,29.3,21.0,21.0,20.6,20.5,20.5,20.3$;

HRMS (ESI+): calcd. for $\left[\mathrm{C}_{27} \mathrm{H}_{33} \mathrm{Cl}_{3} \mathrm{~N}_{2} \mathrm{O}_{12} \mathrm{~K}\right]^{+} 721.0731$, meas. $721.0742, \Delta 1.5 \mathrm{ppm}$. 


\section{$5.8 \quad$ Nucleoside 17}

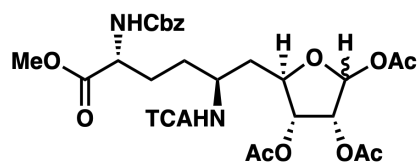

6

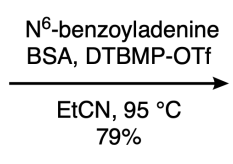

$79 \%$

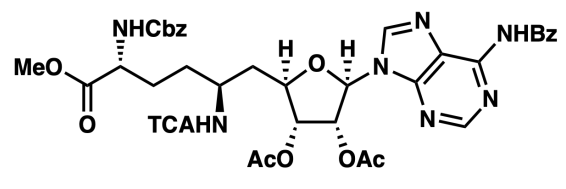

17

To a suspension of $\mathrm{N}^{6}$-benzoyladenine $(168 \mathrm{mg}, 0.702 \mathrm{mmol}, 4.0$ equiv.) in propionitrile (dried over $4 \AA$ M.S., $4.0 \mathrm{~mL})$ at RT, was added $N, O$-bis(trimethylsilyl)acetamide $(0.22 \mathrm{~mL}, 0.88 \mathrm{mmol}, 5.0$ equiv.). The resulting mixture was stirred at $80{ }^{\circ} \mathrm{C}$ for $10 \mathrm{~min}$, upon which complete solubilization of $\mathrm{N}^{6}$-benzoyladenine was observed. To a separate flask charged with triacetate 6 (dried by azeotropic distillation with benzene $(4 \times), 120 \mathrm{mg}, 0.175 \mathrm{mmol})$ and 2,6-di-tert-butyl-4-methylpyridinium triflate (31 $\mathrm{mg}, 88 \mu \mathrm{mol}, 50 \mathrm{~mol} \%)$ was added propionitrile $(4.0 \mathrm{~mL})$. The resulting mixture was stirred at RT for 5 min, until a clear solution was obtained, and the solution of silylated $\mathrm{N}^{6}$-benzoyladenine was added. The reaction mixture was stirred at $95{ }^{\circ} \mathrm{C}$ for $3 \mathrm{~h}$, cooled to $\mathrm{RT}$ and the volatiles were removed in vacuo. Crude material was purified by silica gel chromatography (PhMe/MeCN, $0 \rightarrow 100 \%$ ) to afford nucleoside 17 (119 mg, $0.138 \mathrm{mmol}, 79 \%$ ) as a white amorphous solid.

$\mathrm{R}_{\mathrm{f}}=0.35(\mathrm{EtOAc})$

FTIR (thin film), $\mathrm{cm}^{-1}: \nu_{\max } 3315,2956,1747,1702,1611,1583,1518,1455,1329,1244,1221,1097,1048$;

${ }^{1} \mathrm{H}$ NMR $\left(600 \mathrm{MHz}, \mathrm{CDCl}_{3}\right): \delta 8.77(\mathrm{~s}, 1 \mathrm{H}), 8.11(\mathrm{~s}, 1 \mathrm{H}), 8.06-8.02(\mathrm{~m}, 2 \mathrm{H}), 7.64-7.59(\mathrm{~m}, 1 \mathrm{H}), 7.55-7.51$ $(\mathrm{m}, 2 \mathrm{H}), 7.38-7.28(\mathrm{~m}, 4 \mathrm{H}), 7.11(\mathrm{br} \mathrm{d}, J=8.8 \mathrm{~Hz}, 1 \mathrm{H}), 6.13-6.09(\mathrm{~m}, 2 \mathrm{H}), 5.56(\operatorname{app~t}, J=4.7 \mathrm{~Hz}, 1 \mathrm{H})$, $5.46(\mathrm{br} \mathrm{d}, J=8.3 \mathrm{~Hz}, 1 \mathrm{H}), 5.10(\mathrm{~d}, J=12.9 \mathrm{~Hz}, 1 \mathrm{H}), 5.09(\mathrm{~d}, J=12.9 \mathrm{~Hz}, 1 \mathrm{H}), 4.40-4.33(\mathrm{~m}, 2 \mathrm{H})$, 4.14-4.07 (m, 1H), $3.72(\mathrm{~s}, 3 \mathrm{H}), 2.32(\mathrm{ddd}, J=14.6,11.1,3.4 \mathrm{~Hz}, 1 \mathrm{H}), 2.14(\mathrm{~s}, 3 \mathrm{H}), 2.08(\mathrm{~s}, 3 \mathrm{H}), 2.11-2.05$ $(\mathrm{m}, 1 \mathrm{H}), 1.95-1.86(\mathrm{~m}, 1 \mathrm{H}), 1.77-1.62(\mathrm{~m}, 3 \mathrm{H})$;

${ }^{13} \mathrm{C} \mathrm{NMR}\left(126 \mathrm{MHz}, \mathrm{CDCl}_{3}\right): \delta 172.5,169.9,169.6,164.8,161.8,156.2,153.0,151.6,150.0,142.5,136.2$, 133.5, 133.0, 129.0, 128.7, 128.4, 128.3, 128.1, 124.1, 92.7, 87.7, 80.0, 74.0, 72.5, 67.3, 53.8, 52.7, 49.4, 36.9, $29.8,29.6,20.7,20.5$;

HRMS (ESI+): calcd. for $\left[\mathrm{C}_{37} \mathrm{H}_{39} \mathrm{Cl}_{3} \mathrm{~N}_{7} \mathrm{O}_{11}\right]^{+}$862.1768, meas. 862.1753, $\Delta 1.7 \mathrm{ppm}$. 


\subsection{Amine 18}

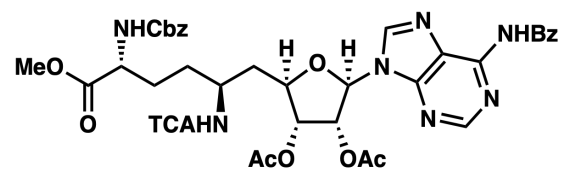

17

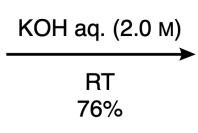

$\%$

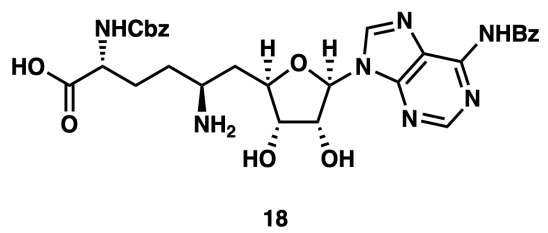

18

To a vial charged with nucleoside $17(167 \mathrm{mg}, 0.193 \mathrm{mmol})$ and cooled to $0{ }^{\circ} \mathrm{C}$, was added a $2.0 \mathrm{M}$ aq. $\mathrm{KOH}$ solution $\left(5.32 \mathrm{~mL}, 10.6 \mathrm{mmol}, 55\right.$ equiv.) precooled to $0{ }^{\circ} \mathrm{C}$. The resulting mixture was stirred at $0{ }^{\circ} \mathrm{C}$ for $5 \mathrm{~h}$ and TFA $(0.60 \mathrm{~mL})$ was added dropwise until $\mathrm{pH} 7$ was reached, at which point a white precipitate appeared. MeCN $(1.5 \mathrm{~mL})$ was added and the resulting clear solution was directly purified by preparative HPLC (gradient elution: $5 \% \rightarrow 45 \%$ B over $30 \mathrm{~min}$ ) to afford amine 18 (108 $\mathrm{mg}, 0.147 \mathrm{mmol}, 76 \%$ ) as the TFA salt.

${ }^{1} \mathrm{H}$ NMR $\left(500 \mathrm{MHz}, \mathbf{C D}_{3} \mathrm{CN}(350 \mu \mathrm{L}) / \mathrm{D}_{2} \mathrm{O}(350 \mu \mathrm{L})\right): \delta 8.75(\mathrm{~s}, 1 \mathrm{H}), 8.56(\mathrm{~s}, 1 \mathrm{H}), 8.04-7.99(\mathrm{~m}, 2 \mathrm{H})$, 7.71-7.65 (m, 1H), 7.59-7.53 (m, 2H), 7.35-7.18 (m, 5H), $6.05(\mathrm{~d}, J=4.2 \mathrm{~Hz}, 1 \mathrm{H}), 5.04-4.96(\mathrm{~m}, 2 \mathrm{H}), 4.71$ $(\mathrm{dd}, J=5.4,4.2 \mathrm{~Hz}, 1 \mathrm{H}), 4.24(\mathrm{dd}, J=5.7,5.4 \mathrm{~Hz}, 1 \mathrm{H}), 4.17(\mathrm{ddd}, J=9.7,5.7,3.0 \mathrm{~Hz}, 1 \mathrm{H}), 4.10-4.03$ $(\mathrm{m}, 1 \mathrm{H}), 3.44-3.34(\mathrm{~m}, 1 \mathrm{H}), 2.15(\mathrm{ddd}, J=14.5,9.7,4.4 \mathrm{~Hz}, 1 \mathrm{H}), 2.09-2.01(\mathrm{~m}, 1 \mathrm{H}), 1.90-1.79(\mathrm{~m}, 1 \mathrm{H})$, $1.78-1.63(\mathrm{~m}, 3 \mathrm{H})$;

${ }^{13} \mathrm{C}$ NMR $\left(126 \mathrm{MHz}, \mathbf{C D}_{3} \mathrm{CN}(350 \mu \mathrm{L}) / \mathrm{D}_{2} \mathrm{O}(350 \mu \mathrm{L})\right): \delta 175.4,169.1,158.1,152.4,150.9,149.0,145.2$, 137.4, 134.7, 133.2, 129.9, 129.5, 129.4, 129.1, 128.5, 123.1, 90.7, 81.1, 74.1, 74.1, 67.6, 54.4, 50.0, 35.4, 29.4, 27.8 ;

HRMS (ESI+): calcd. for $\left[\mathrm{C}_{30} \mathrm{H}_{34} \mathrm{~N}_{7} \mathrm{O}_{8}\right]^{+} 620.2463$, meas. 620.2468, $\Delta 0.8 \mathrm{ppm}$. 


\subsection{Amino Acid S1}
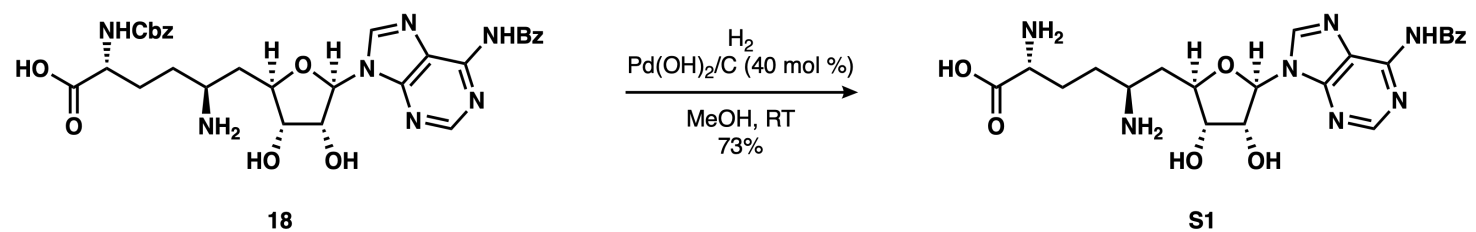

To a solution of $\mathrm{Cbz}$ protected amino acid $18(49 \mathrm{mg}, 67 \mu \mathrm{mol})$ in $\mathrm{MeOH}(6.7 \mathrm{~mL})$ at RT, was added $\mathrm{Pd}(\mathrm{OH})_{2} / \mathrm{C}(20 \mathrm{wt} \%, 19 \mathrm{mg}, 27 \mathrm{\mu mol}, 40 \mathrm{~mol} \%)$. The reaction mixture was purged with hydrogen $(3 \times)$, stirred vigorously at RT under a hydrogen atmosphere (balloon) for $3 \mathrm{~h}$ and filtered through a nylon syringe filter $(13 \mathrm{~mm}, 0.22 \mathrm{\mu m})$. The solids were washed with $\mathrm{MeOH}(5 \times 1 \mathrm{~mL})$ and the filtrates were concentrated. Crude material was purified by preparative HPLC (injection volume: $3.0 \mathrm{~mL}$ (water $/ \mathrm{CH}_{3} \mathrm{CN}, 0.1 \%$ (v/v) TFA, 97.5:2.5), gradient elution: $2.5 \% \rightarrow 40 \% \mathrm{~B}$ over $65 \mathrm{~min}$ ) to afford amino acid $\mathbf{S 1}(35 \mathrm{mg}, 49 \mathrm{\mu mol}$, $73 \%)$ as the TFA salt.

${ }^{1} \mathrm{H}$ NMR $\left(500 \mathrm{MHz}, \mathbf{C D}_{3} \mathrm{CN}(350 \mu \mathrm{L}) / \mathrm{D}_{2} \mathrm{O}(350 \mu \mathrm{L})\right): \delta 8.70(\mathrm{~s}, 1 \mathrm{H}), 8.44(\mathrm{~s}, 1 \mathrm{H}), 8.02-7.96(\mathrm{~m}, 2 \mathrm{H})$, $7.68-7.62(\mathrm{~m}, 1 \mathrm{H}), 7.57-7.52(\mathrm{~m}, 2 \mathrm{H}), 6.03(\mathrm{~d}, J=4.2 \mathrm{~Hz}, 1 \mathrm{H}), 4.70(\mathrm{dd}, J=5.4,4.2 \mathrm{~Hz}, 1 \mathrm{H}), 4.27$ (dd, $J=6.0,5.4 \mathrm{~Hz}, 1 \mathrm{H}), 4.20-4.15(\mathrm{~m}, 1 \mathrm{H}), 3.83(\operatorname{app~t}, J=6.0 \mathrm{~Hz}, 1 \mathrm{H}), 3.49-3.42(\mathrm{~m}, 1 \mathrm{H}), 2.17(\mathrm{ddd}, J=$ $15.4,9.4,4.2 \mathrm{~Hz}, 1 \mathrm{H}), 2.10$ (ddd, $J=15.4,7.5,3.6 \mathrm{~Hz}, 1 \mathrm{H}), 1.97-1.79(\mathrm{~m}, 3 \mathrm{H}), 1.80-1.70(\mathrm{~m}, 1 \mathrm{H})$;

${ }^{13} \mathrm{C}$ NMR $\left(126 \mathrm{MHz}, \mathbf{C D}_{3} \mathrm{CN}(350 \mu \mathrm{L}) / \mathrm{D}_{2} \mathrm{O}(350 \mu \mathrm{L})\right): \delta 172.1,168.9,152.5,151.6,149.4,145.0,134.5$, $133.4,129.9,129.3,123.7,90.6,80.7,74.0,74.0,53.4,49.7,35.0,28.5,26.7$;

HRMS (ESI+): calcd. for $\left[\mathrm{C}_{22} \mathrm{H}_{28} \mathrm{~N}_{7} \mathrm{O}_{6}\right]^{+} 486.2096$, meas. 486.2098, $\Delta 0.5 \mathrm{ppm}$. 


\subsection{C9'-epi-sinefungin (5)}
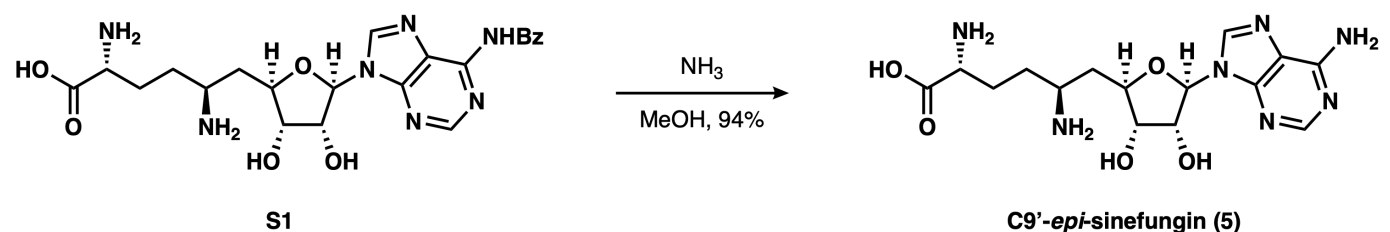

C9'-epi-sinefungin (5)

To a vial charged with Bz protected C9'-epi-sinefungin S1 (35 mg, $49 \mu \mathrm{mol}$ ) was added a solution of ammonia in methanol $(7 \mathrm{~N}, 5 \mathrm{~mL})$ at RT. The resulting mixture was stirred for $5.5 \mathrm{~h}$ and the volatiles were removed in vacuo. Crude material was purified by preparative HPLC (injection volume: $3.0 \mathrm{~mL}$ (water), elution: $2.5 \%$ B over $20 \mathrm{~min}$ ) to afford C9'-epi-sinefungin (5) (28 mg, $46 \mu \mathrm{mol}, 94 \%$ ) as the TFA salt. Analytical data is presented in section 6 .

${ }^{1} \mathrm{H}$ NMR $\left(500 \mathrm{MHz}, \mathrm{D}_{2} \mathrm{O}(875 \mu \mathrm{L}\right.$ for $19 \mathrm{mg}$ of material)): $\delta 8.46(\mathrm{~s}, 1 \mathrm{H}), 8.46(\mathrm{~s}, 1 \mathrm{H}), 6.14(\mathrm{~d}, J=4.1 \mathrm{~Hz}$, $1 \mathrm{H}), 4.80(\mathrm{dd}, J=5.5,4.1 \mathrm{~Hz}, 1 \mathrm{H}), 4.39(\mathrm{dd}, J=6.0,5.5 \mathrm{~Hz}, 1 \mathrm{H}), 4.30(\mathrm{ddd}, J=9.6,6.0,3.7 \mathrm{~Hz}, 1 \mathrm{H})$, 3.90 (app t, $J=5.9 \mathrm{~Hz}, 1 \mathrm{H}), 3.62-3.56(\mathrm{~m}, 1 \mathrm{H}), 2.32-2.19(\mathrm{~m}, 2 \mathrm{H}), 2.07-1.82(\mathrm{~m}, 4 \mathrm{H})$;

${ }^{13} \mathrm{C}$ NMR (126 MHz, $\mathrm{D}_{2} \mathrm{O}$ (875 $\mu \mathrm{L}$ for $19 \mathrm{mg}$ of material)): $\delta 173.2,150.3,148.1,145.0,142.9,119.1,89.3$, $79.7,73.2,73.1,53.8,48.8,34.2,27.7,26.2$;

HRMS (ESI+): calcd. for $\left[\mathrm{C}_{15} \mathrm{H}_{24} \mathrm{~N}_{7} \mathrm{O}_{5}\right]^{+} 382.1833$, meas. 382.1827, $\Delta 1.6 \mathrm{ppm}$. 


\subsection{Benzamide 19}

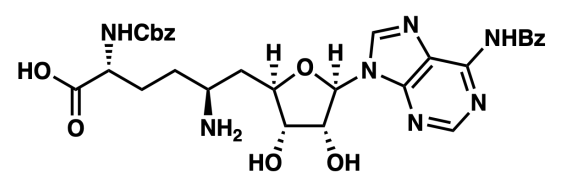

18
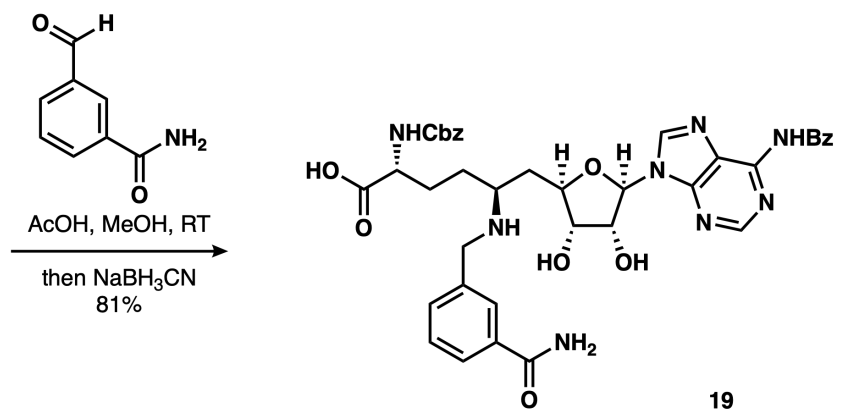

To a solution of amine 18 (TFA salt, $50 \mathrm{mg}, 0.068 \mathrm{mmol}$ ) in $\mathrm{MeOH}(4.0 \mathrm{~mL}$ ) at RT, were added 3formyl-benzamide (203 mg, $1.36 \mathrm{mmol}, 20$ equiv.) and $\mathrm{AcOH}(40 \mu \mathrm{L}, 0.68 \mathrm{mmol}, 10$ equiv.). The resulting mixture was stirred at RT for $1.5 \mathrm{~h}$ before the addition of $\mathrm{NaBH}_{3} \mathrm{CN}(1.0 \mathrm{M}$ in THF, $3.4 \mathrm{~mL}, 3.4 \mathrm{mmol}, 50$ equiv.). The reaction mixture was stirred for $4 \mathrm{~h}$ and the volatiles were removed in vacuo. Crude material was purified by preparative HPLC (gradient elution: $5 \% \rightarrow 45 \%$ B over $60 \mathrm{~min}$ ) to afford benzamide 19 (48 $\mathrm{mg}, 0.055 \mathrm{mmol}, 81 \%)$ as the TFA salt.

${ }^{1} \mathrm{H}$ NMR $\left(600 \mathrm{MHz}, \mathbf{C D}_{3} \mathrm{CN}(350 \mu \mathrm{L}) / \mathrm{D}_{2} \mathrm{O}(350 \mu \mathrm{L})\right): \delta 8.54(\mathrm{~s}, 1 \mathrm{H}), 8.44(\mathrm{~s}, 1 \mathrm{H}), 8.02-7.99(\mathrm{~m}, 2 \mathrm{H})$, 7.69-7.65 (m, 1H), $7.62(\mathrm{dt}, J=6.5,2.0 \mathrm{~Hz}, 1 \mathrm{H}), 7.60-7.54(\mathrm{~m}, 3 \mathrm{H}), 7.32-7.21(\mathrm{~m}, 7 \mathrm{H}), 5.96(\mathrm{~d}, J=4.4$ $\mathrm{Hz}, 1 \mathrm{H}), 5.02(\mathrm{~d}, J=14.6 \mathrm{~Hz}, 1 \mathrm{H}), 5.02(\mathrm{~d}, J=14.6 \mathrm{~Hz}, 1 \mathrm{H}), 4.66(\mathrm{dd}, J=5.5,4.4 \mathrm{~Hz}, 1 \mathrm{H}), 4.29(\mathrm{dd}, J$ $=6.1,5.5 \mathrm{~Hz}, 1 \mathrm{H}), 4.21-4.18(\mathrm{~m}, 1 \mathrm{H}), 4.14-4.10(\mathrm{~m}, 1 \mathrm{H}), 4.11(\mathrm{~d}, J=13.5 \mathrm{~Hz}, 1 \mathrm{H}), 4.07(\mathrm{~d}, J=13.5 \mathrm{~Hz}$, $1 \mathrm{H}), 3.51-3.44(\mathrm{~m}, 1 \mathrm{H}), 2.31-2.18(\mathrm{~m}, 2 \mathrm{H}), 1.91-1.70(\mathrm{~m}, 4 \mathrm{H})$;

${ }^{13} \mathrm{C}$ NMR $\left(126 \mathrm{MHz}, \mathbf{C D}_{3} \mathrm{CN}(350 \mu \mathrm{L}) / \mathrm{D}_{2} \mathrm{O}(350 \mu \mathrm{L})\right): \delta 175.3,171.1,168.3,158.1,152.6,152.1,150.2$, 144.8, 137.4, 134.4, 134.2, 133.9, 133.7, 132.0, 130.1, 129.8, 129.5, 129.4, 129.2, 129.1, 129.1, 128.6, 124.5, $90.4,80.7,73.7,73.4,67.6,56.4,54.3,48.6,32.0,27.9,26.7$;

HRMS (ESI+): calcd. for $\left[\mathrm{C}_{38} \mathrm{H}_{41} \mathrm{~N}_{8} \mathrm{O}_{9}\right]^{+} 753.2991$, meas. $753.3014, \Delta 3.1 \mathrm{ppm}$. 


\section{Analytical Characterization of C9'-epi-Sinefungin}

\section{Method Description}

Detection of various compounds by LC/MS were carried out on a Thermo Scientific Dionex UltiMate 3000 UHPLC coupled to a Thermo Q Exactive Plus mass spectrometer system (Thermo Fisher Scientific Inc, Waltham, MA) equipped with an HESI-II electrospray ionization (ESI) source. Data were acquired with Chromeleon Xpress software for UHPLC and Thermo Xcalibur software version 3.0.63 for mass spectrometry, and processed with Thermo Xcalibur Qual Browser software version 4.0.27.19.

$1 \mu \mathrm{L}$ sample was injected onto the UHPLC including an HPG-3400RS binary pump with a built-in vacuum degasser and a thermostated WPS-3000TRS high performance autosampler. A Coagent Diamond Hydride analytical column $(2.1 \times 150 \mathrm{~mm}, 4 \mu \mathrm{m})$ from Microsolv Technology Corporation (Leland, NC) was used at the flow rate of $0.4 \mathrm{~mL} / \mathrm{min}$ using $0.1 \%$ formic acid in water as mobile phase $\mathrm{A}$ and $0.1 \%$ formic acid in acetonitrile as mobile phase B. The column temperature was maintained at room temperature. The following gradient was applied: 0-5 min: $95-75 \% \mathrm{~B}, 5-6 \mathrm{~min}: 75-60 \% \mathrm{~B}, 6-7 \mathrm{~min}$ : $60 \%$ B isocratic, 7-9 min: 60-50\% B, 9-15 min: 50\% B isocratic, 15-16 min: 50-95\% B, 16-25 min, 95\%B isocratic.

The MS conditions were as follows: positive ionization mode; full scan mass range, m/z 50 to 750; resolution, 70,000; AGC target, 1e6; maximum IT, 200 ms; PRM NCE, 20 for m/z 382.1833; resolution, 35,000; AGC target, 2e5; maximum IT, $100 \mathrm{~ms}$; spray voltage, $3500 \mathrm{~V}$; capillary temperature, $280{ }^{\circ} \mathrm{C}$; sheath gas, 50; Aux gas, 12.5; probe heater temperature, $425{ }^{\circ} \mathrm{C}$; S-Lens RF level, 50.00. A mass window of $\pm 5 \mathrm{ppm}$ was used to extract the ion of $[\mathrm{M}+\mathrm{H}]^{+}$for the compounds. Targets were considered detected when the mass

accuracy was less than $5 \mathrm{ppm}$ and there were a match of isotopic pattern between the observed and the theoretical ones. 


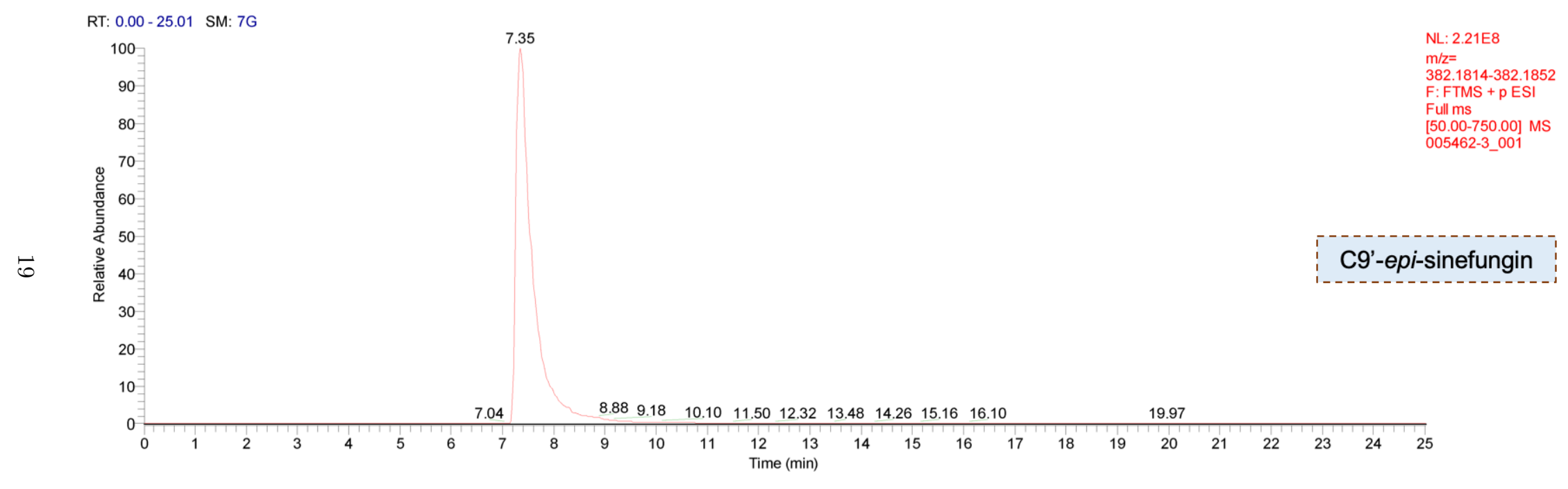

LC/MS Compound Detection 


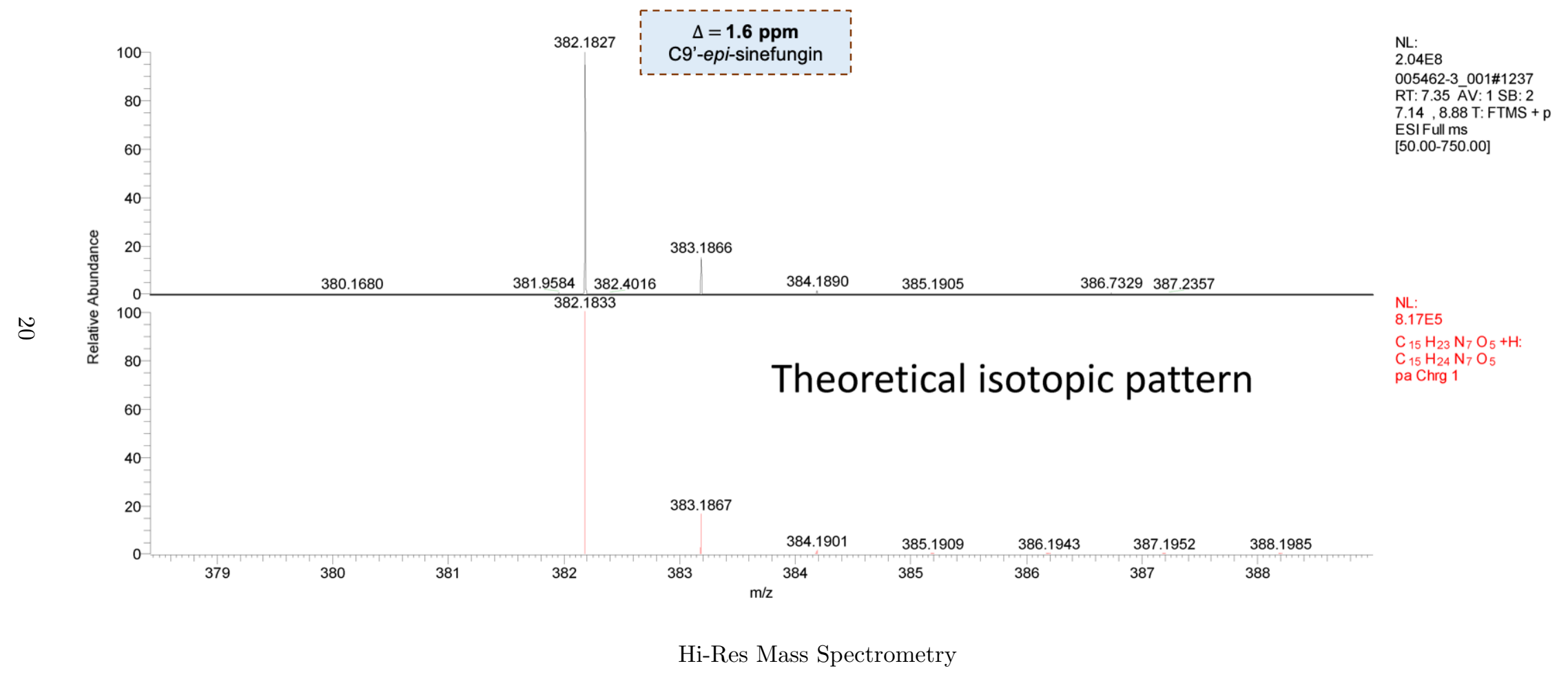




\section{Small-Molecule X-Ray Crystallography}

A crystal mounted on a diffractometer was collected data at $100 \mathrm{~K}$. The intensities of the reflections were collected by means of a Bruker APEX DUO CCD diffractometer $\left(\mathrm{Cu}_{\mathrm{K} \alpha}\right.$ radiation, $\left.\lambda=1.54178 \AA\right)$, and equipped with an Oxford Cryosystems nitrogen flow apparatus. The collection method involved $1.0^{\circ}$ scans in $\omega$ at $-30^{\circ},-55^{\circ},-80^{\circ}, 30^{\circ}, 55^{\circ}, 80^{\circ}$ and $115^{\circ}$ in $2 \theta$. Data integration down to $0.84 \AA$ resolution was carried out using SAINT V8.37 $\mathrm{A}^{10}$ with reflection spot size optimization. Absorption corrections were made with the program SADABS ${ }^{10}$. The structure was solved by the Intrinsic Phasing methods and refined by least-squares methods again $F^{2}$ using SHELXT-2014 11 and SHELXL-2014 ${ }^{12}$ with OLEX 2 interface ${ }^{13}$. Non-hydrogen atoms were refined anisotropically, and hydrogen atoms were allowed to ride on the respective atoms. Crystal data as well as details of data collection and refinement are summarized in Table 1 for compound 12. Geometric parameters are shown in Table 2, and hydrogen-bond parameters are listed in Table 3. The Ortep plots were produced with SHELXL-2014, and the other images were produced with Accelrys DS Visualizer $2.0^{14}$.

\section{$7.1 \quad(R)$-Carbamate 12}

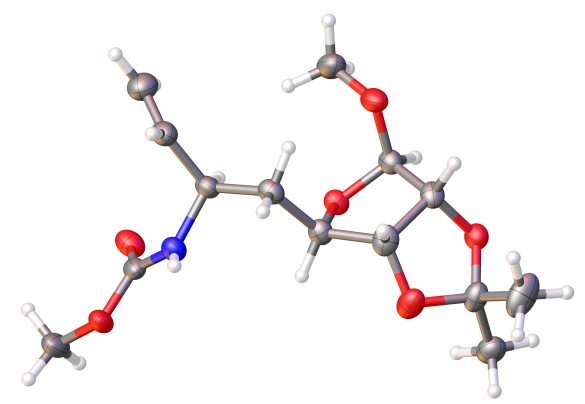

Table 1: Experimental Details

\begin{tabular}{|l|l|}
\hline Crystal Data & \\
\hline Chemical Formula & $\mathrm{C}_{14} \mathrm{H}_{23} \mathrm{NO}_{6}$ \\
\hline$M_{r}$ & 301.33 \\
\hline Crystal system, space group & Monoclinic, $C 2$ \\
\hline Temperature $(\mathrm{K})$ & 100 \\
\hline$a, b, c(\AA)$ & $26.9198(7), 5.4862(1), 10.6405(3)$ \\
\hline$\beta\left(^{\circ}\right)$ & $99.4349(14)$ \\
\hline$V\left(\AA^{3}\right)$ & $1550.21(7)$ \\
\hline
\end{tabular}

${ }^{10}$ Bruker AXS APEX3, Bruker AXS, Madison, Wisconsin, 2015.

11 Sheldrick, G. M. Acta Crystallogr., Sect. A 2015, 71, 3-8.

${ }^{12}$ Sheldrick, G. M. Acta Crystallogr., Sect. C 2015, 71, 3-8.

${ }^{13}$ Dolomanov, O. V.; Bourhis, L. J.; Gildea, R. J.; Howard, J. A. K.; Puschmann, H. J. Appl. Cryst. 2009, 42, 339-341.

${ }^{14}$ Accelrys DS Visualizer v2.0.1, Accelrys Software Inc., 2007. 


\begin{tabular}{|c|c|}
\hline$Z$ & 4 \\
\hline Radiation type & $\mathrm{Cu} K \alpha$ \\
\hline$\mu\left(\mathrm{mm}^{-1}\right)$ & 0.84 \\
\hline Crystal size (mm) & $0.18 \times 0.12 \times 0.06$ \\
\hline \multicolumn{2}{|l|}{ Data collection } \\
\hline Diffractometer & Bruker D8 goniometer with CCD area detector \\
\hline Absorption correction & Multi-scan $S A D A B S$ \\
\hline$T_{\min }, T_{\max }$ & $0.724,0.806$ \\
\hline $\begin{array}{l}\text { No. of measured, independent and } \\
\text { observed }[I>2 \sigma(I)] \text { reflections }\end{array}$ & $12976,2622,2557$ \\
\hline$R_{\text {int }}$ & 0.027 \\
\hline$(\sin \theta / \lambda)_{\max }\left(\AA^{-1}\right)$ & 0.596 \\
\hline \multicolumn{2}{|l|}{ Refinement } \\
\hline$R\left[F^{2}>2 \sigma\left(F^{2}\right)\right], w R\left(F^{2}\right), S$ & $0.033,0.087,1.05$ \\
\hline No. of reflections & 2622 \\
\hline No. of parameters & 198 \\
\hline No. of restraints & 1 \\
\hline H-atom treatment & $\begin{array}{l}\mathrm{H} \text { atoms treated by a mixture of independent and constrained } \\
\text { refinement }\end{array}$ \\
\hline$\Delta \rho_{\max }, \Delta \rho_{\min }\left(e \AA^{-3}\right)$ & $0.39,-0.14$ \\
\hline Absolute structure & Flack x determined using 1093 quotients $[(\mathrm{I}+)-(\mathrm{I}-)] /[(\mathrm{I}+)+(\mathrm{I}-)]^{15}$ \\
\hline Absolute structure parameter & $-0.07(17)$ \\
\hline
\end{tabular}

Computer programs: SAINT 8.37A (Bruker-AXS, 2015), SHELXT2014 (Sheldrick, 2015), SHELXL2014 (Sheldrick, 2015), Bruker SHELXTL (Sheldrick, 2015).

Table 2: Geometric parameters $\left(\AA{ }^{\circ}\right)$

\begin{tabular}{|l|l|l|l|}
\hline O1-C1 & $1.426(3)$ & C5-H5 & 1 \\
\hline O1-C2 & $1.433(3)$ & C6-C7 & $1.533(4)$ \\
\hline O2-C3 & $1.418(3)$ & C6-H6A & 0.99 \\
\hline O2-C1 & $1.428(3)$ & C6-H6B & 0.99 \\
\hline O3-C4 & $1.408(3)$ & C7-C8 & $1.504(3)$ \\
\hline O3-C5 & $1.450(3)$ & C7-H7 & 1 \\
\hline O4-C4 & $1.412(3)$ & C8-C9 & $1.311(4)$ \\
\hline O4-C12 & $1.427(4)$ & C8-H8 & 0.95 \\
\hline O5-C13 & $1.354(3)$ & C9-H9A & 0.95 \\
\hline O5-C14 & $1.440(4)$ & C9-H9B & 0.95 \\
\hline O6-C13 & $1.210(3)$ & C10-H10A & 0.98 \\
\hline
\end{tabular}

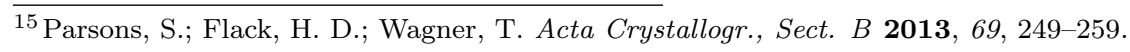




\begin{tabular}{|c|c|c|c|}
\hline N1-C13 & $1.348(4)$ & C10-H10B & 0.98 \\
\hline N1-C7 & $1.469(3)$ & C10-H10C & 0.98 \\
\hline $\mathrm{N} 1-\mathrm{H} 1$ & $0.81(4)$ & C11-H11A & 0.98 \\
\hline $\mathrm{C} 1-\mathrm{C} 11$ & $1.503(4)$ & C11-H11B & 0.98 \\
\hline $\mathrm{C} 1-\mathrm{C} 10$ & $1.513(4)$ & C11-H11C & 0.98 \\
\hline $\mathrm{C} 2-\mathrm{C} 5$ & $1.530(4)$ & $\mathrm{C} 12-\mathrm{H} 12 \mathrm{~A}$ & 0.98 \\
\hline $\mathrm{C} 2-\mathrm{C} 3$ & $1.543(4)$ & $\mathrm{C} 12-\mathrm{H} 12 \mathrm{~B}$ & 0.98 \\
\hline $\mathrm{C} 2-\mathrm{H} 2$ & 1 & $\mathrm{C} 12-\mathrm{H} 12 \mathrm{C}$ & 0.98 \\
\hline C3-C4 & $1.514(4)$ & C14-H14A & 0.98 \\
\hline C3-H3 & 1 & C14-H14B & 0.98 \\
\hline $\mathrm{C} 4-\mathrm{H} 4$ & 1 & C14-H14C & 0.98 \\
\hline $\mathrm{C} 5-\mathrm{C} 6$ & $1.522(4)$ & & \\
\hline $\mathrm{C} 1-\mathrm{O} 1-\mathrm{C} 2$ & $107.8(2)$ & C7-C6-H6B & 109.4 \\
\hline C3-O2-C1 & $107.72(18)$ & $\mathrm{H} 6 \mathrm{~A}-\mathrm{C} 6-\mathrm{H} 6 \mathrm{~B}$ & 108 \\
\hline C4-O3-C5 & $108.53(17)$ & $\mathrm{N} 1-\mathrm{C} 7-\mathrm{C} 8$ & $110.3(2)$ \\
\hline $\mathrm{C} 4-\mathrm{O} 4-\mathrm{C} 12$ & $112.3(2)$ & $\mathrm{N} 1-\mathrm{C} 7-\mathrm{C} 6$ & $109.1(2)$ \\
\hline C13-O5-C14 & $114.9(2)$ & $\mathrm{C} 8-\mathrm{C} 7-\mathrm{C} 6$ & $113.2(2)$ \\
\hline C13-N1-C7 & $120.9(2)$ & N1-C7-H7 & 108 \\
\hline C13-N1-H1 & $121(2)$ & $\mathrm{C} 8-\mathrm{C} 7-\mathrm{H} 7$ & 108 \\
\hline C7-N1-H1 & $117(2)$ & $\mathrm{C} 6-\mathrm{C} 7-\mathrm{H} 7$ & 108 \\
\hline $\mathrm{O} 1-\mathrm{C} 1-\mathrm{O} 2$ & $104.2(2)$ & $\mathrm{C} 9-\mathrm{C} 8-\mathrm{C} 7$ & $124.2(3)$ \\
\hline O1-C1-C11 & $108.8(2)$ & C9-C8-H8 & 117.9 \\
\hline $\mathrm{O} 2-\mathrm{C} 1-\mathrm{C} 11$ & $109.1(2)$ & $\mathrm{C} 7-\mathrm{C} 8-\mathrm{H} 8$ & 117.9 \\
\hline O1-C1-C10 & $111.8(2)$ & C8-C9-H9A & 120 \\
\hline $\mathrm{O} 2-\mathrm{C} 1-\mathrm{C} 10$ & $110.6(3)$ & C8-C9-H9B & 120 \\
\hline $\mathrm{C} 11-\mathrm{C} 1-\mathrm{C} 10$ & $112.2(3)$ & H9A-C9-H9B & 120 \\
\hline $\mathrm{O} 1-\mathrm{C} 2-\mathrm{C} 5$ & $109.8(2)$ & C1-C10-H10A & 109.5 \\
\hline $\mathrm{O} 1-\mathrm{C} 2-\mathrm{C} 3$ & $104.21(19)$ & C1-C10-H10B & 109.5 \\
\hline C5-C2-C3 & $105.0(2)$ & H10A-C10-H10B & 109.5 \\
\hline $\mathrm{O} 1-\mathrm{C} 2-\mathrm{H} 2$ & 112.4 & C1-C10-H10C & 109.5 \\
\hline $\mathrm{C} 5-\mathrm{C} 2-\mathrm{H} 2$ & 112.4 & $\mathrm{H} 10 \mathrm{~A}-\mathrm{C} 10-\mathrm{H} 10 \mathrm{C}$ & 109.5 \\
\hline $\mathrm{C} 3-\mathrm{C} 2-\mathrm{H} 2$ & 112.4 & H10B-C10-H10C & 109.5 \\
\hline $\mathrm{O} 2-\mathrm{C} 3-\mathrm{C} 4$ & $109.10(19)$ & $\mathrm{C} 1-\mathrm{C} 11-\mathrm{H} 11 \mathrm{~A}$ & 109.5 \\
\hline $\mathrm{O} 2-\mathrm{C} 3-\mathrm{C} 2$ & $104.5(2)$ & C1-C11-H11B & 109.5 \\
\hline C4-C3-C2 & $104.5(2)$ & H11A-C11-H11B & 109.5 \\
\hline $\mathrm{O} 2-\mathrm{C} 3-\mathrm{H} 3$ & 112.7 & C1-C11-H11C & 109.5 \\
\hline C4-C3-H3 & 112.7 & $\mathrm{H} 11 \mathrm{~A}-\mathrm{C} 11-\mathrm{H} 11 \mathrm{C}$ & 109.5 \\
\hline C2-C3-H3 & 112.7 & $\mathrm{H} 11 \mathrm{~B}-\mathrm{C} 11-\mathrm{H} 11 \mathrm{C}$ & 109.5 \\
\hline O3-C4-O4 & $113.24(19)$ & $\mathrm{O} 4-\mathrm{C} 12-\mathrm{H} 12 \mathrm{~A}$ & 109.5 \\
\hline $\mathrm{O} 3-\mathrm{C} 4-\mathrm{C} 3$ & $106.11(19)$ & $\mathrm{O} 4-\mathrm{C} 12-\mathrm{H} 12 \mathrm{~B}$ & 109.5 \\
\hline
\end{tabular}




\begin{tabular}{|c|c|c|c|}
\hline $\mathrm{O} 4-\mathrm{C} 4-\mathrm{C} 3$ & $107.6(2)$ & H12A-C12-H12B & 109.5 \\
\hline O3-C4-H4 & 109.9 & $\mathrm{O} 4-\mathrm{C} 12-\mathrm{H} 12 \mathrm{C}$ & 109.5 \\
\hline $\mathrm{O} 4-\mathrm{C} 4-\mathrm{H} 4$ & 109.9 & $\mathrm{H} 12 \mathrm{~A}-\mathrm{C} 12-\mathrm{H} 12 \mathrm{C}$ & 109.5 \\
\hline $\mathrm{C} 3-\mathrm{C} 4-\mathrm{H} 4$ & 109.9 & H12B-C12-H12C & 109.5 \\
\hline $\mathrm{O} 3-\mathrm{C} 5-\mathrm{C} 6$ & $110.78(19)$ & $\mathrm{O} 6-\mathrm{C} 13-\mathrm{N} 1$ & $125.8(2)$ \\
\hline $\mathrm{O} 3-\mathrm{C} 5-\mathrm{C} 2$ & $104.39(18)$ & $\mathrm{O} 6-\mathrm{C} 13-\mathrm{O} 5$ & $124.2(2)$ \\
\hline $\mathrm{C} 6-\mathrm{C} 5-\mathrm{C} 2$ & $115.5(2)$ & N1-C13-O5 & $110.0(2)$ \\
\hline O3-C5-H5 & 108.7 & O5-C14-H14A & 109.5 \\
\hline C6-C5-H5 & 108.7 & O5-C14-H14B & 109.5 \\
\hline $\mathrm{C} 2-\mathrm{C} 5-\mathrm{H} 5$ & 108.7 & H14A-C14-H14B & 109.5 \\
\hline $\mathrm{C} 5-\mathrm{C} 6-\mathrm{C} 7$ & $111.1(2)$ & O5-C14-H14C & 109.5 \\
\hline C5-C6-H6A & 109.4 & $\mathrm{H} 14 \mathrm{~A}-\mathrm{C} 14-\mathrm{H} 14 \mathrm{C}$ & 109.5 \\
\hline C7-C6-H6A & 109.4 & H14B-C14-H14C & 109.5 \\
\hline C5-C6-H6B & 109.4 & & \\
\hline $\mathrm{C} 2-\mathrm{O} 1-\mathrm{C} 1-\mathrm{O} 2$ & $-32.3(3)$ & $\mathrm{O} 2-\mathrm{C} 3-\mathrm{C} 4-\mathrm{O} 4$ & $-148.49(18)$ \\
\hline $\mathrm{C} 2-\mathrm{O} 1-\mathrm{C} 1-\mathrm{C} 11$ & $-148.5(2)$ & $\mathrm{C} 2-\mathrm{C} 3-\mathrm{C} 4-\mathrm{O} 4$ & $100.2(2)$ \\
\hline $\mathrm{C} 2-\mathrm{O} 1-\mathrm{C} 1-\mathrm{C} 10$ & $87.1(3)$ & $\mathrm{C} 4-\mathrm{O} 3-\mathrm{C} 5-\mathrm{C} 6$ & $92.0(2)$ \\
\hline $\mathrm{C} 3-\mathrm{O} 2-\mathrm{C} 1-\mathrm{O} 1$ & $34.1(3)$ & $\mathrm{C} 4-\mathrm{O} 3-\mathrm{C} 5-\mathrm{C} 2$ & $-32.9(2)$ \\
\hline C3-O2-C1-C11 & $150.1(2)$ & O1-C2-C5-O3 & $-93.7(2)$ \\
\hline $\mathrm{C} 3-\mathrm{O} 2-\mathrm{C} 1-\mathrm{C} 10$ & $-86.1(3)$ & $\mathrm{C} 3-\mathrm{C} 2-\mathrm{C} 5-\mathrm{O} 3$ & $17.9(2)$ \\
\hline $\mathrm{C} 1-\mathrm{O} 1-\mathrm{C} 2-\mathrm{C} 5$ & $130.3(2)$ & $\mathrm{O} 1-\mathrm{C} 2-\mathrm{C} 5-\mathrm{C} 6$ & $144.5(2)$ \\
\hline $\mathrm{C} 1-\mathrm{O} 1-\mathrm{C} 2-\mathrm{C} 3$ & $18.3(3)$ & C3-C2-C5-C6 & $-104.0(2)$ \\
\hline $\mathrm{C} 1-\mathrm{O} 2-\mathrm{C} 3-\mathrm{C} 4$ & $-133.7(2)$ & $\mathrm{O} 3-\mathrm{C} 5-\mathrm{C} 6-\mathrm{C} 7$ & $62.4(2)$ \\
\hline $\mathrm{C} 1-\mathrm{O} 2-\mathrm{C} 3-\mathrm{C} 2$ & $-22.4(2)$ & $\mathrm{C} 2-\mathrm{C} 5-\mathrm{C} 6-\mathrm{C} 7$ & $-179.23(19)$ \\
\hline $\mathrm{O} 1-\mathrm{C} 2-\mathrm{C} 3-\mathrm{O} 2$ & $2.5(2)$ & C13-N1-C7-C8 & $84.5(3)$ \\
\hline $\mathrm{C} 5-\mathrm{C} 2-\mathrm{C} 3-\mathrm{O} 2$ & $-113.0(2)$ & C13-N1-C7-C6 & $-150.5(2)$ \\
\hline $\mathrm{O} 1-\mathrm{C} 2-\mathrm{C} 3-\mathrm{C} 4$ & $117.1(2)$ & $\mathrm{C} 5-\mathrm{C} 6-\mathrm{C} 7-\mathrm{N} 1$ & $73.0(3)$ \\
\hline $\mathrm{C} 5-\mathrm{C} 2-\mathrm{C} 3-\mathrm{C} 4$ & $1.6(2)$ & C5-C6-C7-C8 & $-163.7(2)$ \\
\hline C5-O3-C4-O4 & $-83.3(2)$ & N1-C7-C8-C9 & $-114.5(3)$ \\
\hline $\mathrm{C} 5-\mathrm{O} 3-\mathrm{C} 4-\mathrm{C} 3$ & $34.5(2)$ & C6-C7-C8-C9 & $122.9(3)$ \\
\hline C12-O4-C4-O3 & $-59.3(3)$ & $\mathrm{C} 7-\mathrm{N} 1-\mathrm{C} 13-\mathrm{O} 6$ & $10.7(4)$ \\
\hline $\mathrm{C} 12-\mathrm{O} 4-\mathrm{C} 4-\mathrm{C} 3$ & $-176.2(2)$ & $\mathrm{C} 7-\mathrm{N} 1-\mathrm{C} 13-\mathrm{O} 5$ & $-169.5(2)$ \\
\hline $\mathrm{O} 2-\mathrm{C} 3-\mathrm{C} 4-\mathrm{O} 3$ & $90.0(2)$ & $\mathrm{C} 14-\mathrm{O} 5-\mathrm{C} 13-\mathrm{O} 6$ & $-2.5(4)$ \\
\hline $\mathrm{C} 2-\mathrm{C} 3-\mathrm{C} 4-\mathrm{O} 3$ & $-21.3(2)$ & $\mathrm{C} 14-\mathrm{O} 5-\mathrm{C} 13-\mathrm{N} 1$ & $177.8(2)$ \\
\hline
\end{tabular}

Table 3: Hydrogen-bond parameters

\begin{tabular}{|l|l|l|l|l|}
\hline $\mathrm{D}-\mathrm{H} \cdots A$ & $\mathrm{D}-\mathrm{H}(\AA)$ & $\mathrm{H} \cdots A(\AA)$ & $\mathrm{D} \cdots A(\AA)$ & $\mathrm{D}-\mathrm{H} \cdots A\left(^{\circ}\right)$ \\
\hline $\mathrm{C} 14-\mathrm{H} 14 \mathrm{C} \cdots \mathrm{O} 1^{\mathrm{i}}$ & 0.98 & 2.77 & $3.136(3)$ & 102.7 \\
\hline
\end{tabular}

Symmetry code(s): (i) $-x+1, y-1,-z+1$. 


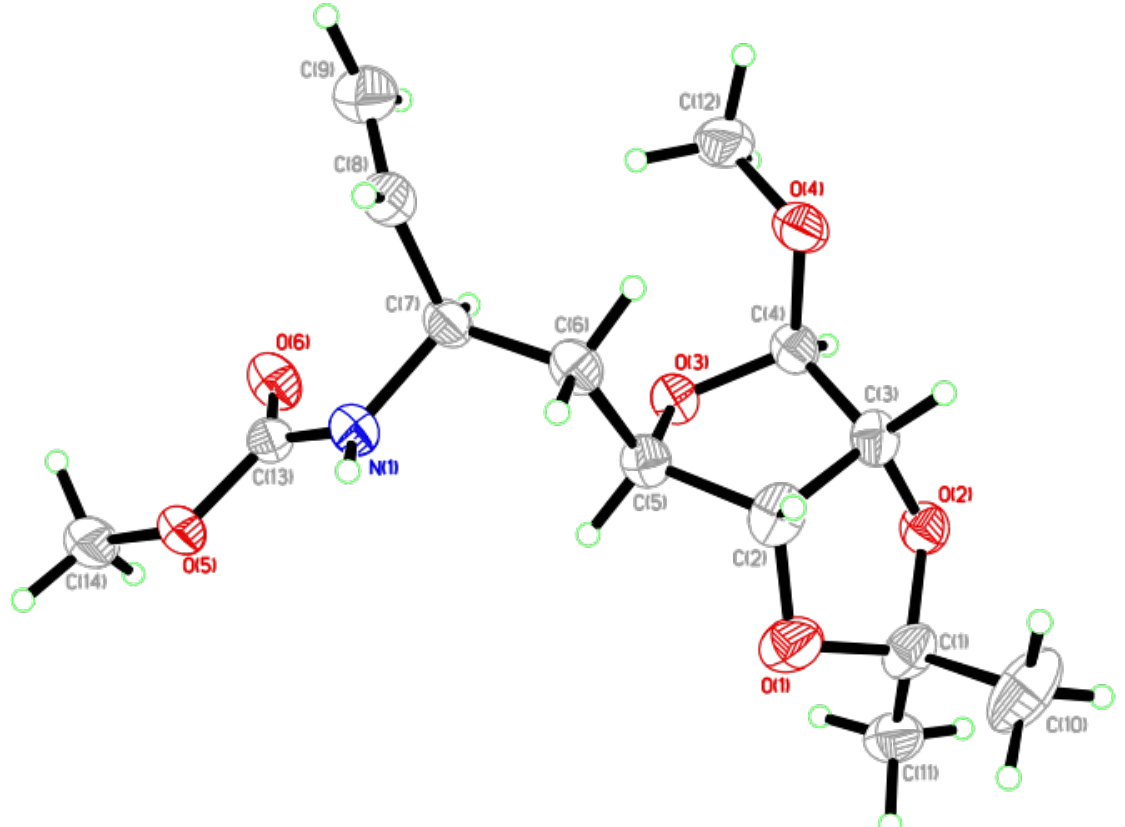

Figure 1: Perspective views showing $50 \%$ probability displacement.

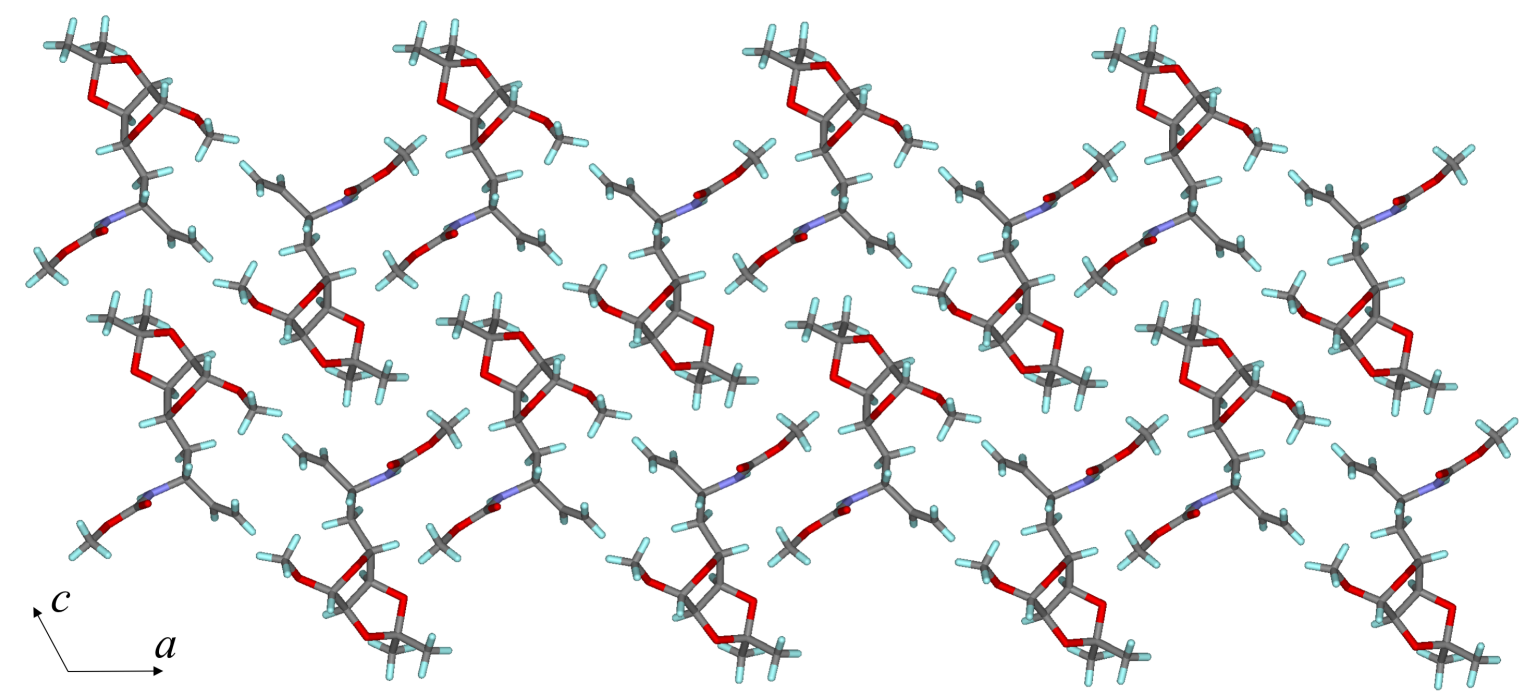

Figure 2: Three-dimensional supramolecular architecture viewed along the $b$-axis direction. 


\section{NMR Spectra}

8 Trichloroacetimidate . . . . . . . . . . . . . . . . . . . . . . 26

$7(R)$-Trichloroacetamide . . . . . . . . . . . . . . . . . . . . . . . . . . 29

$13(S)$-Trichloroacetamide . . . . . . . . . . . . . . . . . . . . . . . . . . . . . 31

14 Aldehyde . . . . . . . . . . . . . . . . . . . . . . . . . 33

15 Enamide . . . . . . . . . . . . . . . . . . . . . . . . . . 35

16 Protected Amino Acid . . . . . . . . . . . . . . . . . . . . . . . . . 37

6 Triacetate . . . . . . . . . . . . . . . . . . . . . . . . . . 39

17 Nucleoside . . . . . . . . . . . . . . . . . . . . . . . . . . 41

18 Amine . . . . . . . . . . . . . . . . . . . . . . . . . . . . 43

S1 Amino Acid . . . . . . . . . . . . . . . . . . . . . . . . . . 45

5 C9'-epi-sinefungin . . . . . . . . . . . . . . . . . . . . . . . . 47

19 Benzamide . . . . . . . . . . . . . . . . . . . . . . . . . 49 


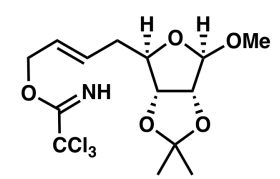

8, ${ }^{1} \mathrm{H}, 600 \mathrm{MHz}, \mathrm{CDCl}_{3}$

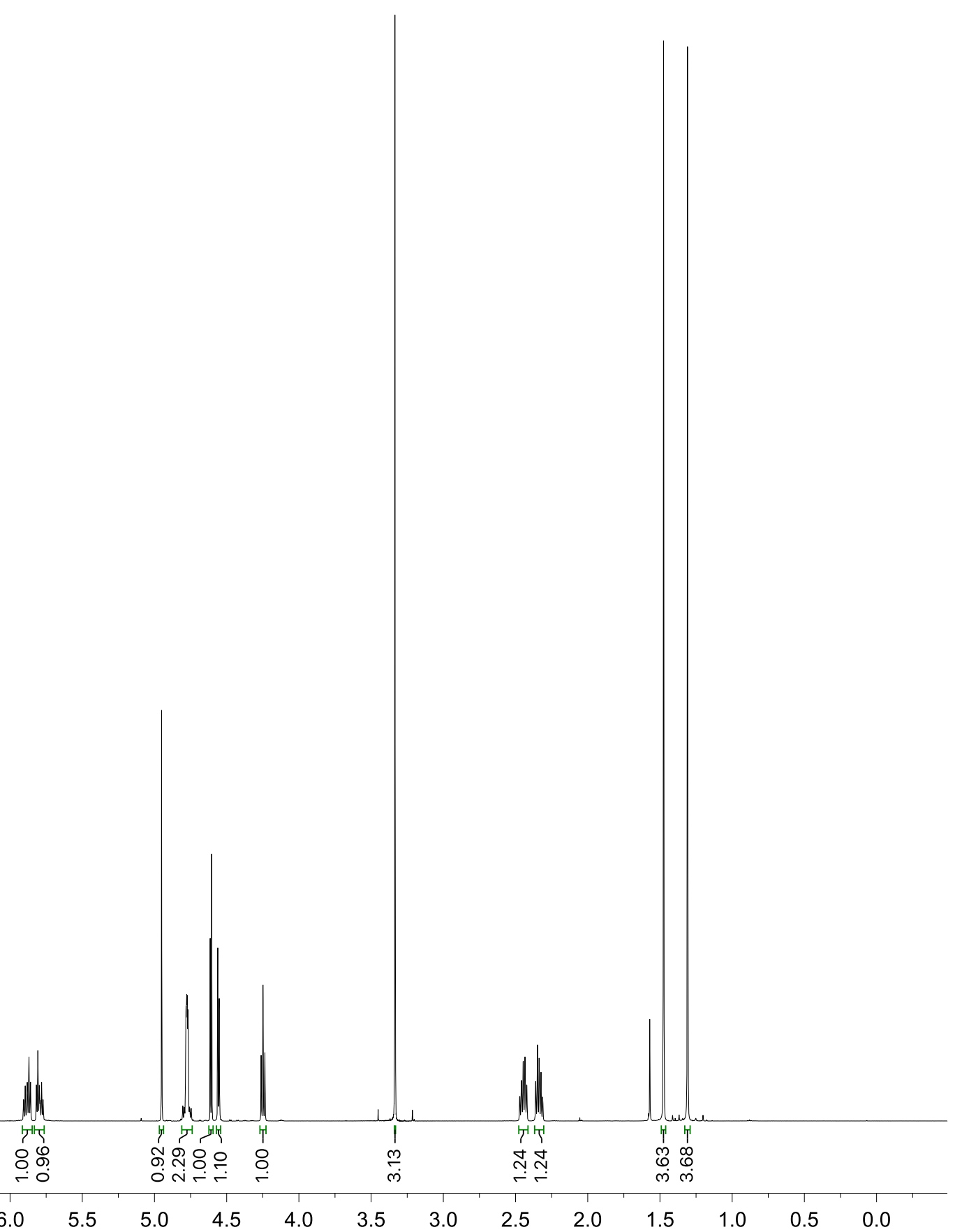



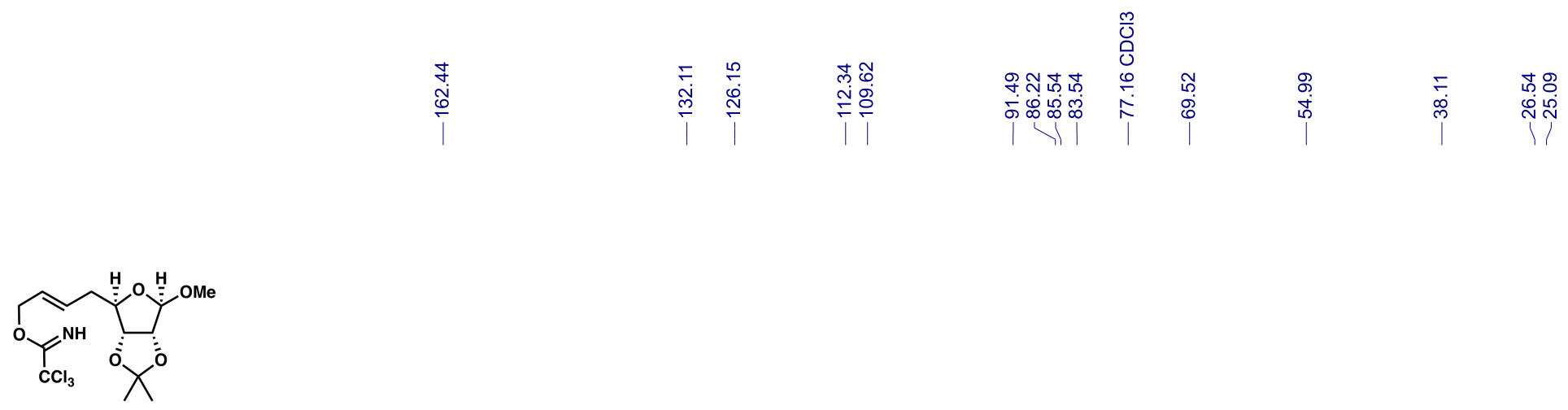

8, ${ }^{13} \mathrm{C}, 126 \mathrm{MHz}, \mathrm{CDCl}_{3}$

$\stackrel{\infty}{\infty}$

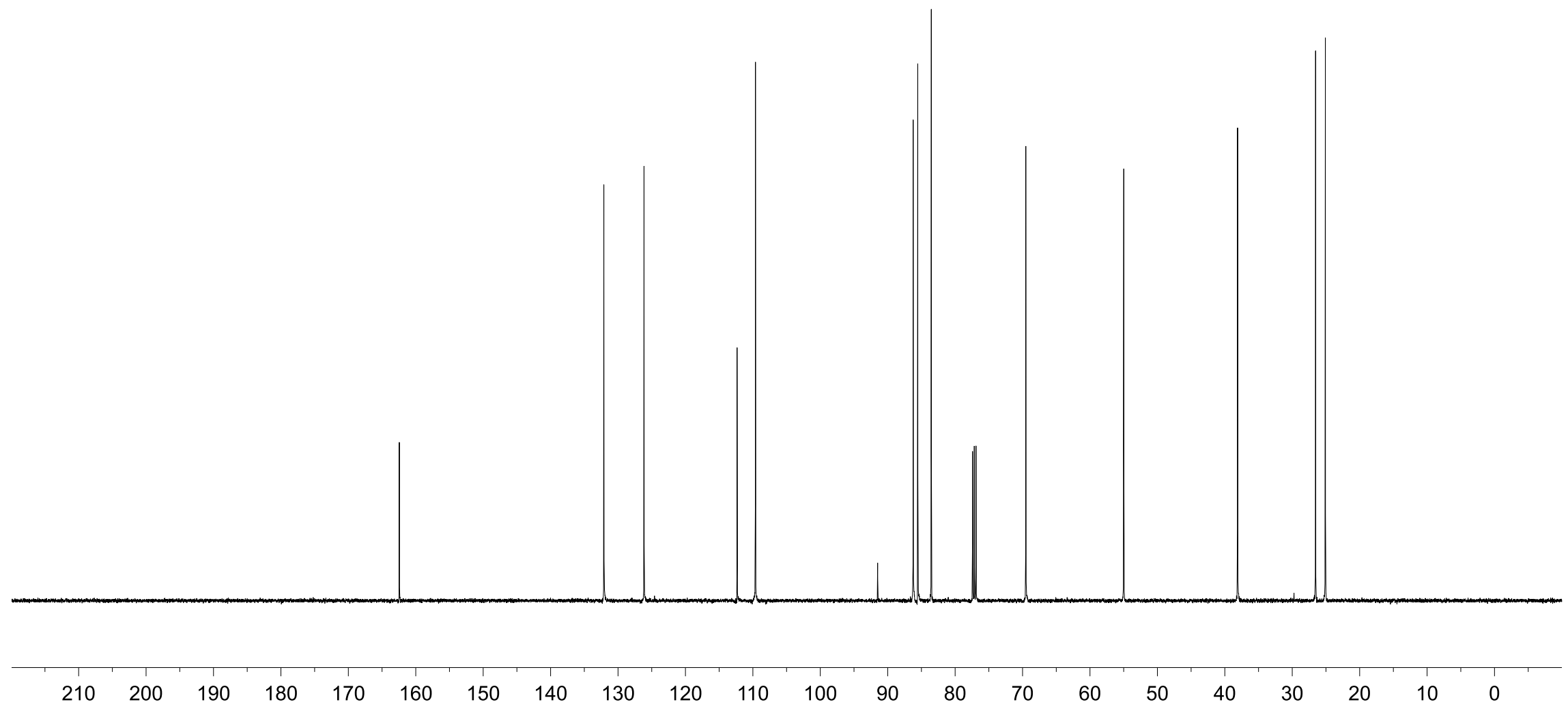


7, ${ }^{1} \mathrm{H}, 500 \mathrm{MHz}, \mathrm{CDCl}_{3}$

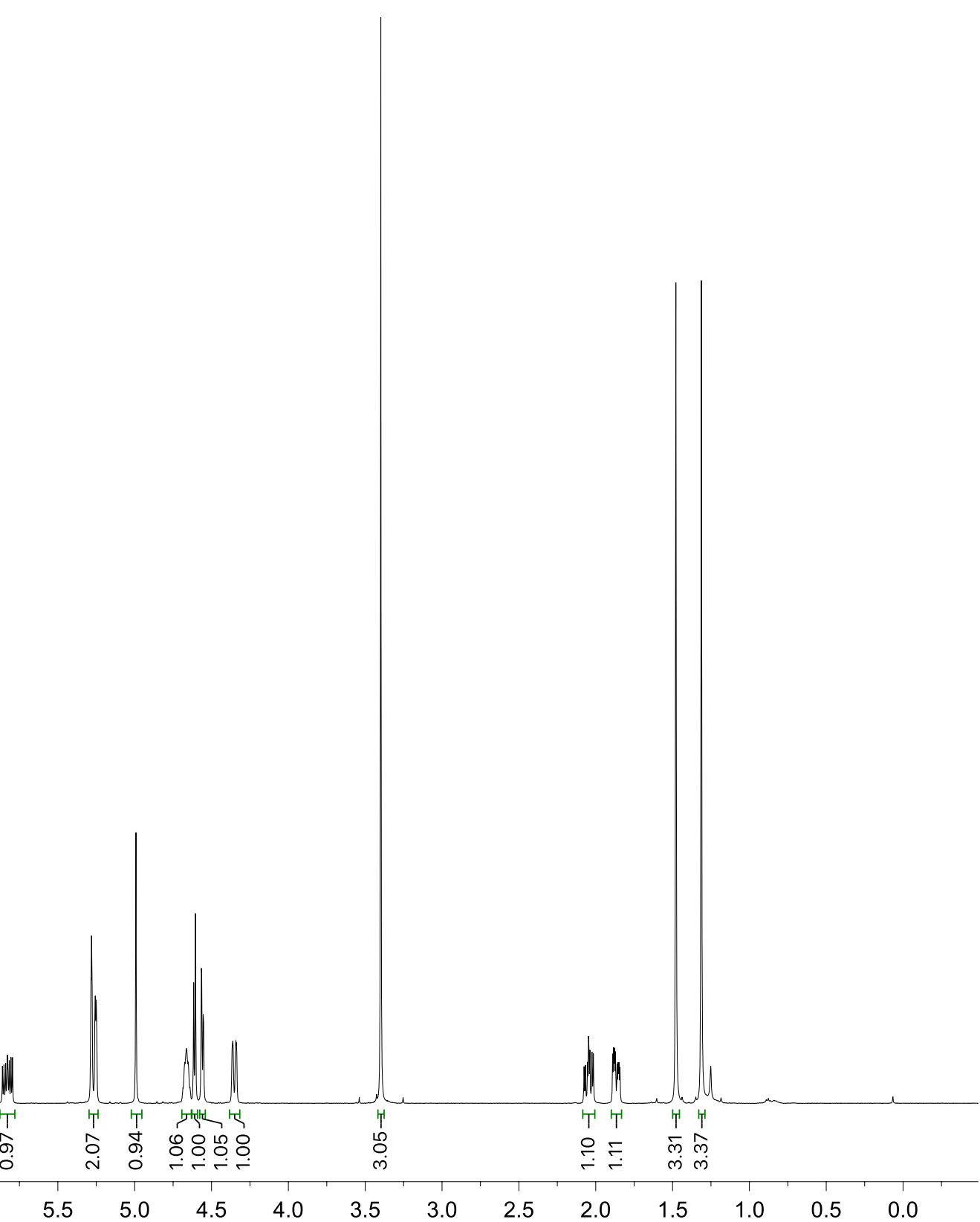


理 


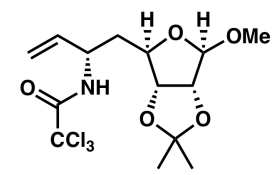

13, ${ }^{1} \mathrm{H}, 500 \mathrm{MHz}, \mathrm{CDCl}_{3}$

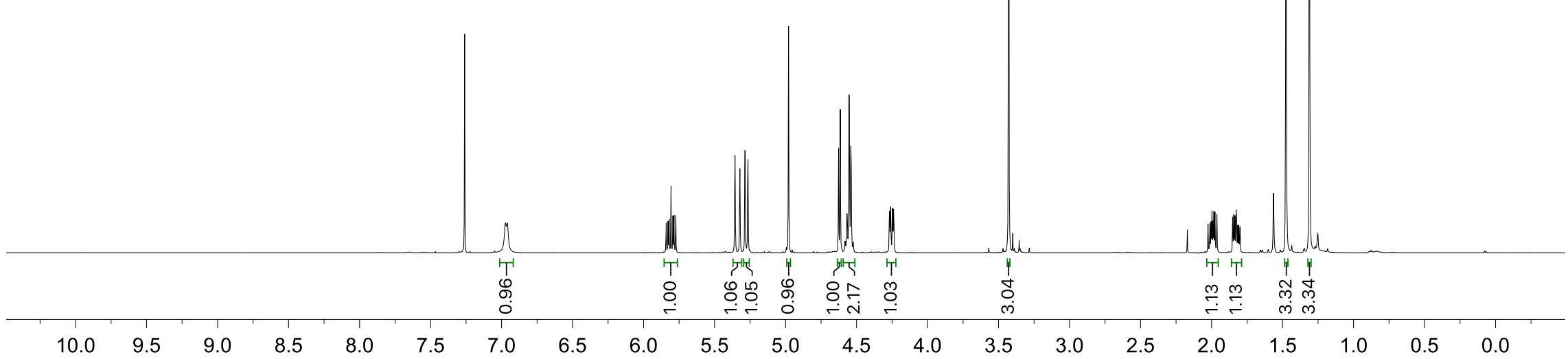




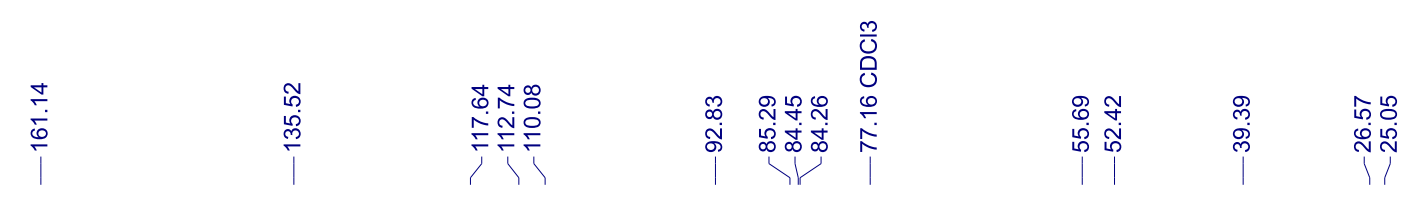

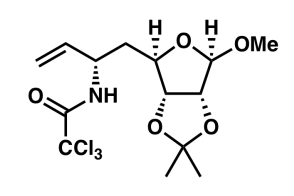

13, ${ }^{13} \mathrm{C}, 126 \mathrm{MHz}, \mathrm{CDCl}_{3}$

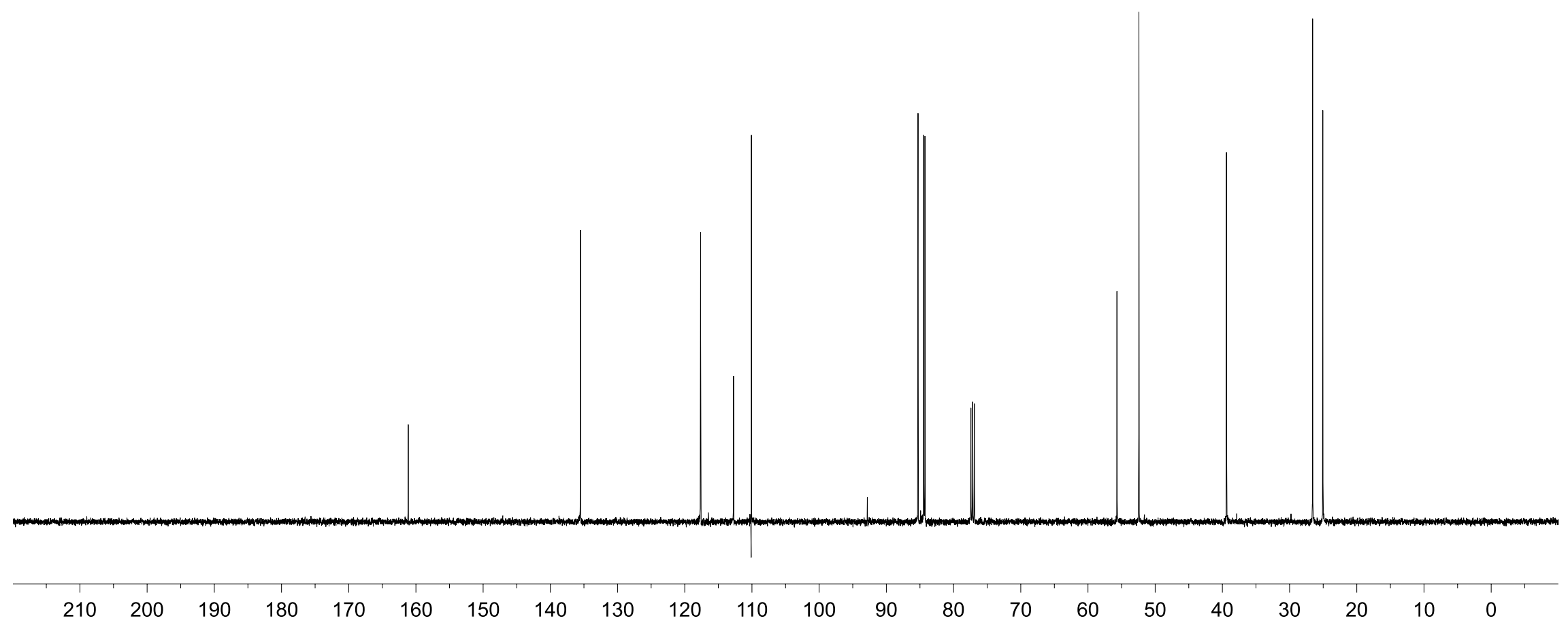




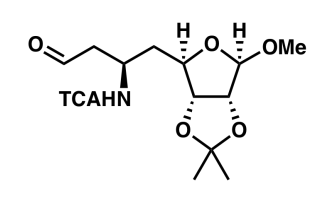

14, ${ }^{1} \mathrm{H}, 500 \mathrm{MHz}, \mathrm{CDCl}_{3}$

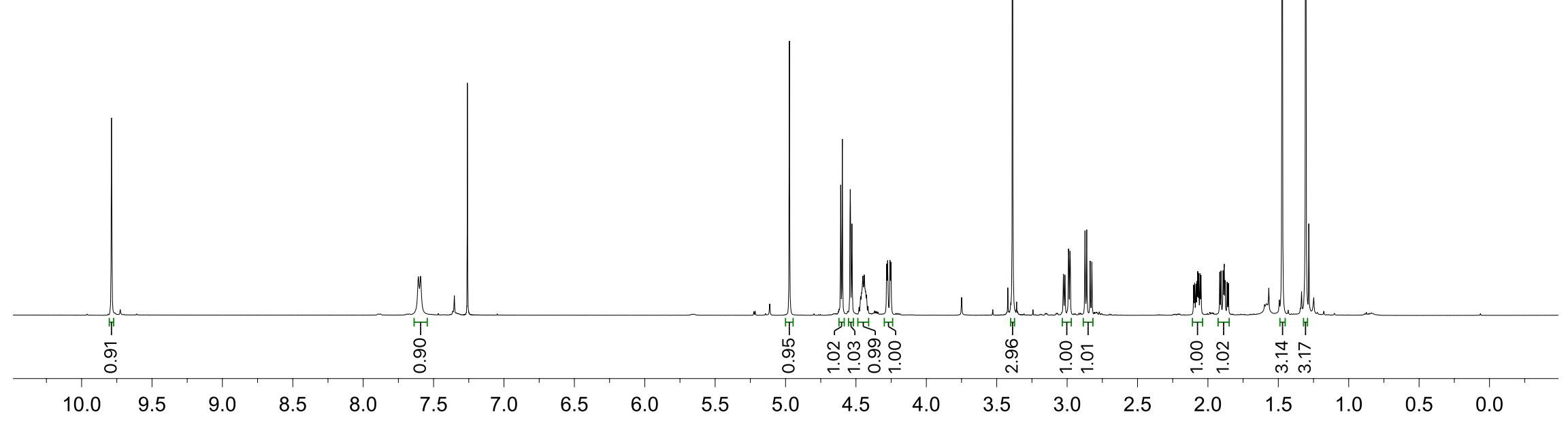




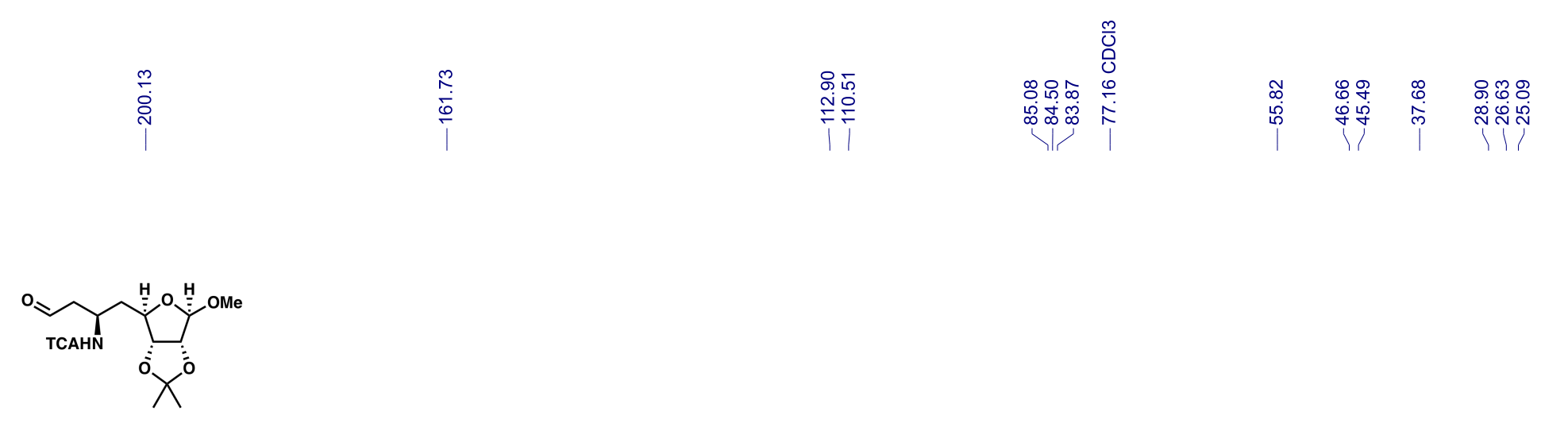

$\mathbf{1 4},{ }^{13} \mathrm{C}, 126 \mathrm{MHz}, \mathrm{CDCl}_{3}$

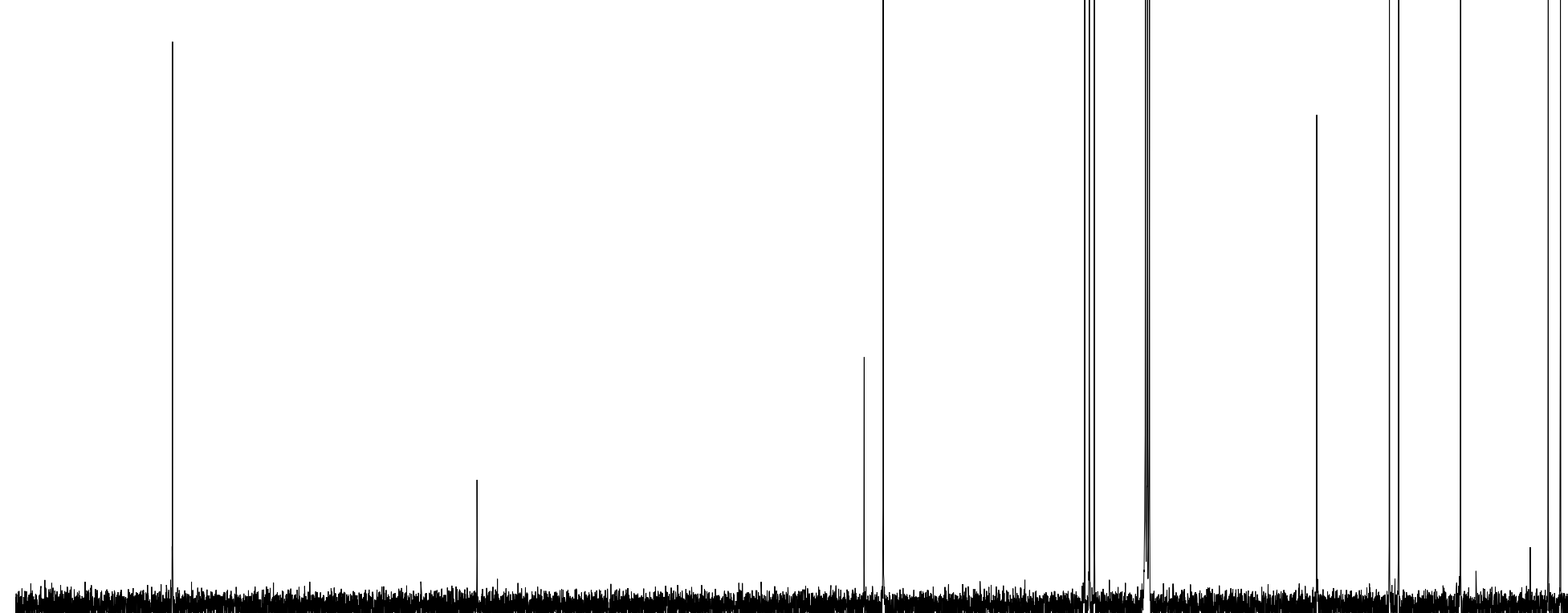

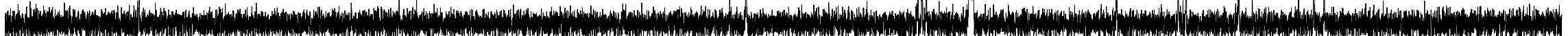




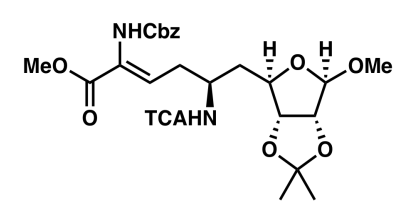

15, ${ }^{1} \mathrm{H}, 500 \mathrm{MHz}, \mathrm{CDCl}_{3}$

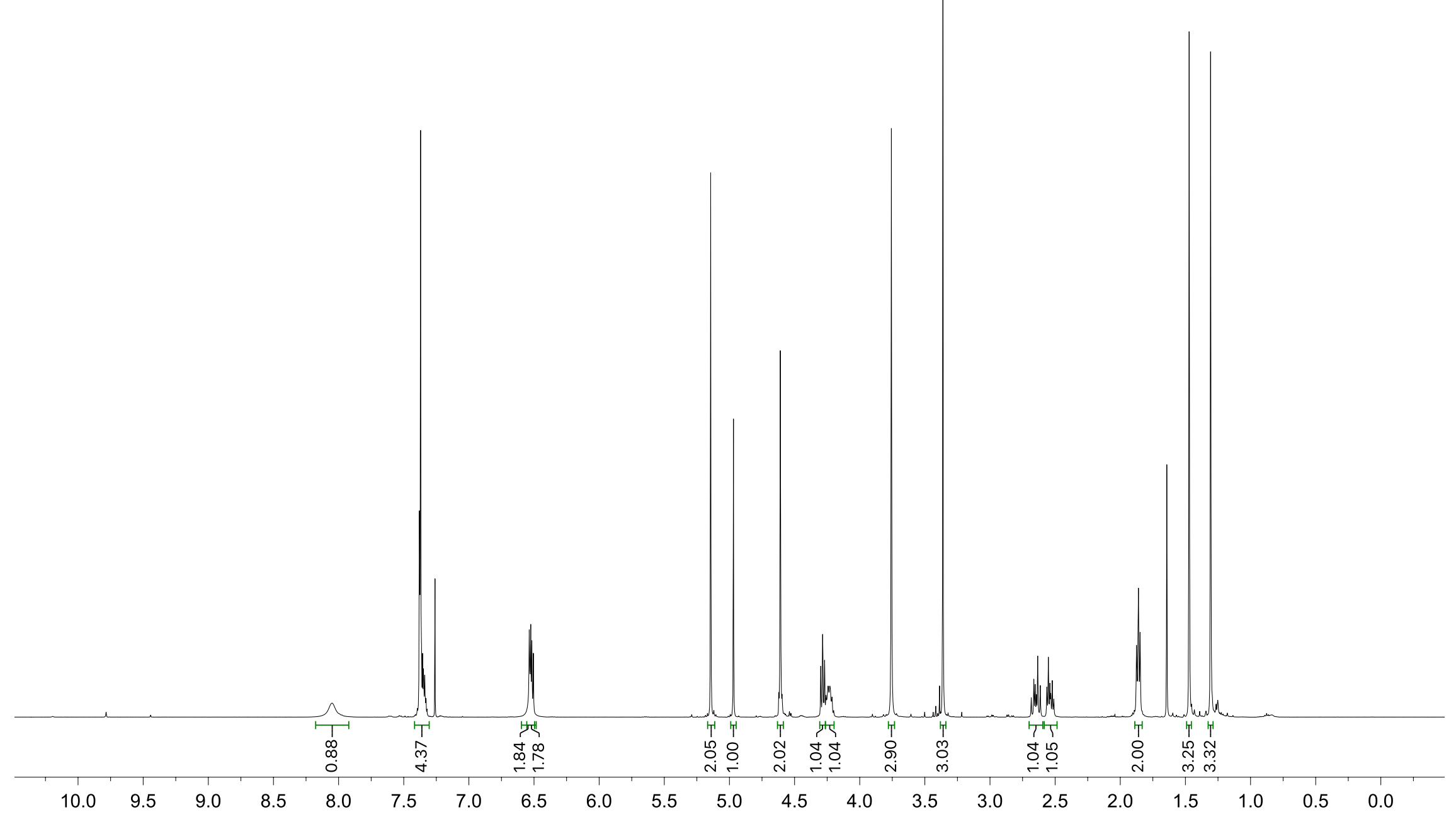




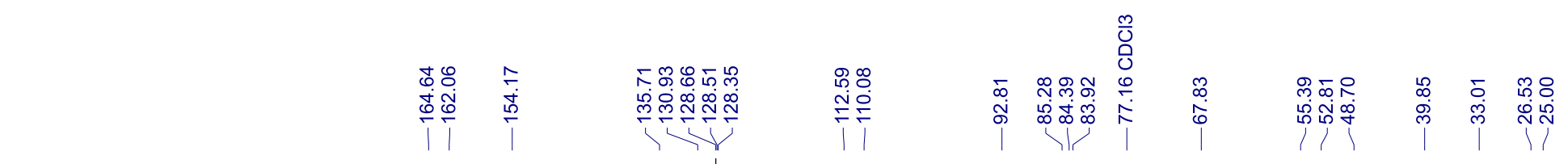

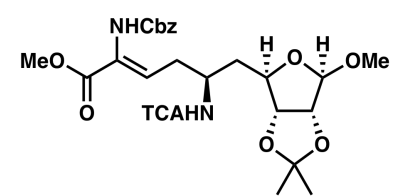

$\mathbf{1 5},{ }^{13} \mathrm{C}, 151 \mathrm{MHz}, \mathrm{CDCl}_{3}$

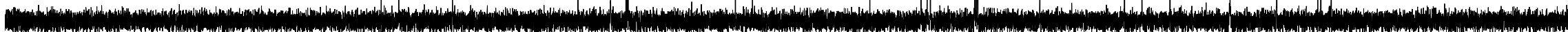




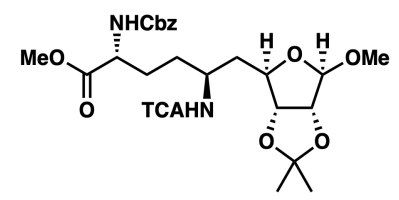

16, ${ }^{1} \mathrm{H}, 600 \mathrm{MHz}, \mathrm{CDCl}_{3}$

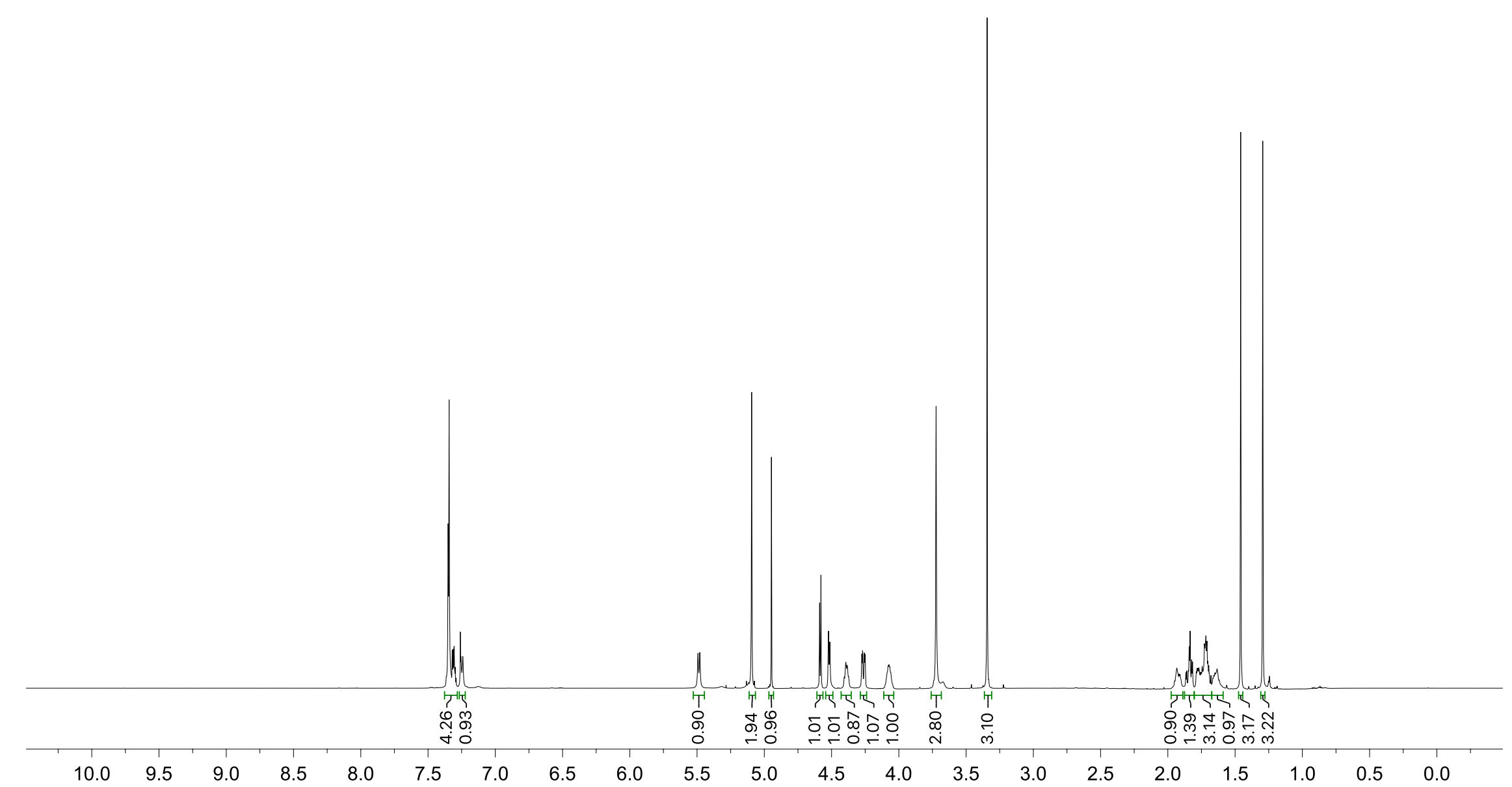




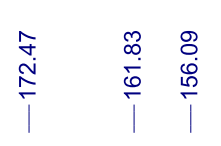
엉

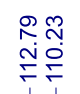
ะิ

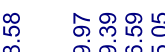

$\overbrace{\text { O TCAHN }}^{\text {MeO }}$

16, ${ }^{13} \mathrm{C}, 151 \mathrm{MHz}, \mathrm{CDCl}_{3}$ 


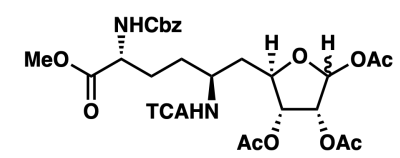

6, ${ }^{1} \mathrm{H}, 600 \mathrm{MHz}, \mathrm{CDCl}_{3}$

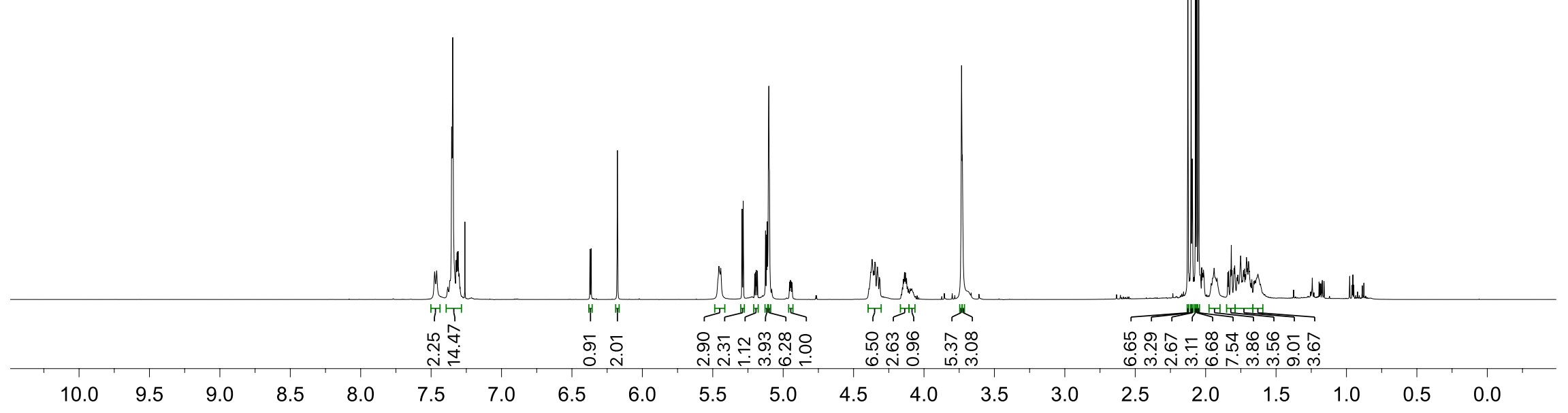




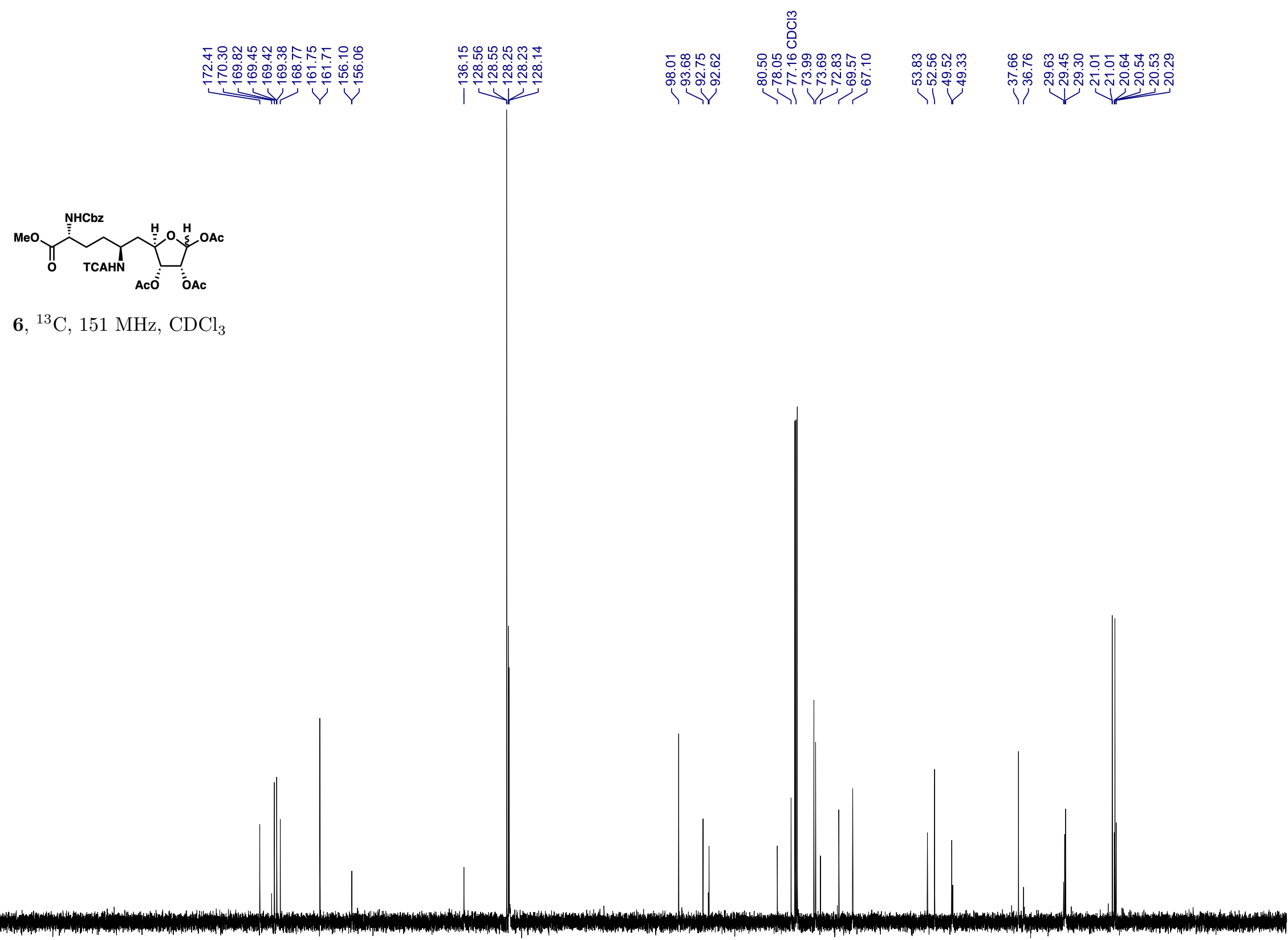

$210 \quad 200$

190

180

$170 \quad 160$

150

140

130

120

110

100

90

80

70

60

50

$40 \quad 30$

$20 \quad 10$ 


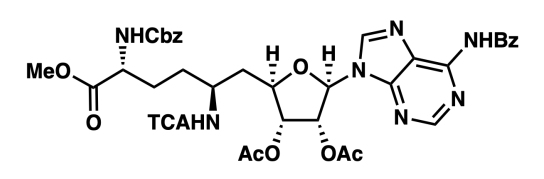

17, ${ }^{1} \mathrm{H}, 600 \mathrm{MHz}, \mathrm{CDCl}_{3}$

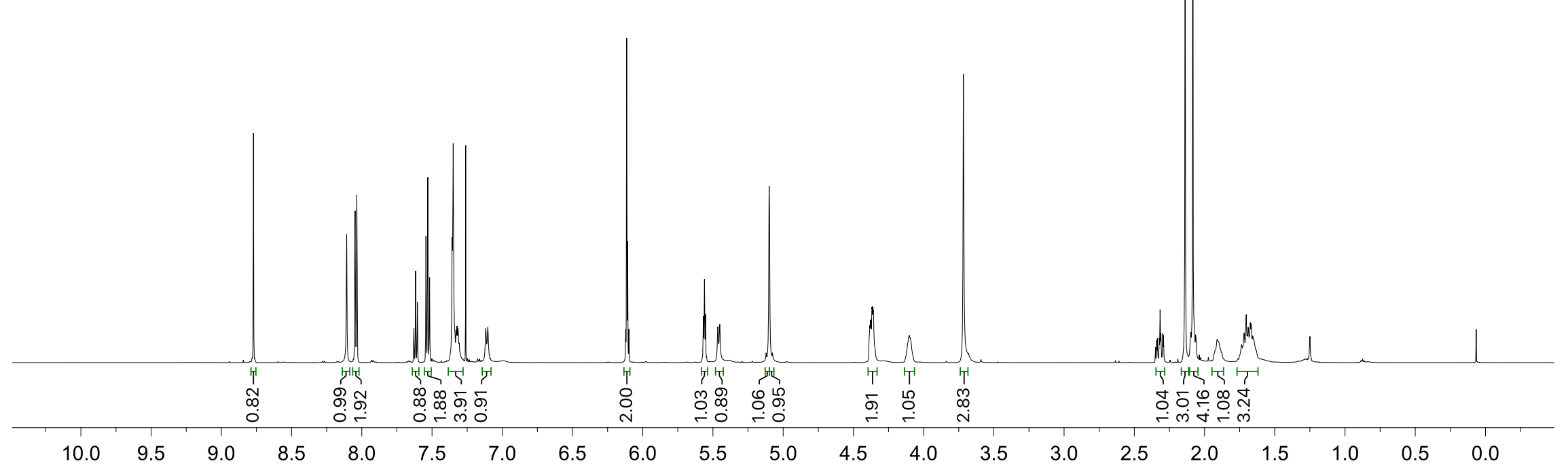




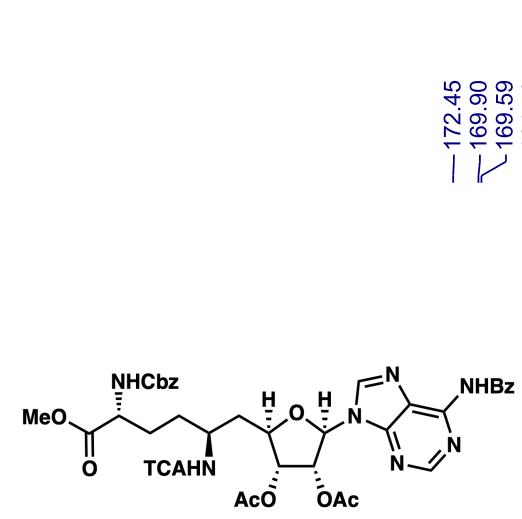

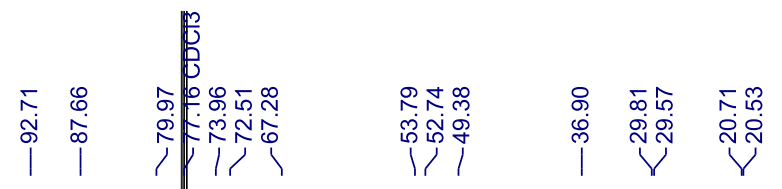

17, ${ }^{13} \mathrm{C}, 126 \mathrm{MHz}, \mathrm{CDCl}_{3}$

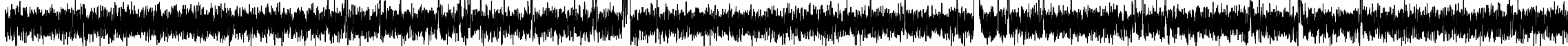




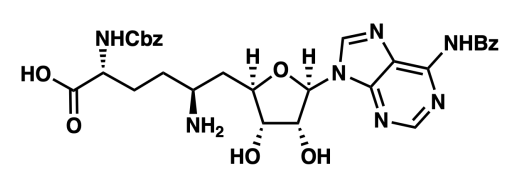

18, ${ }^{1} \mathrm{H}, 500 \mathrm{MHz}, \mathbf{C D}_{\mathbf{3}} \mathbf{C N} / \mathrm{D}_{2} \mathrm{O}$

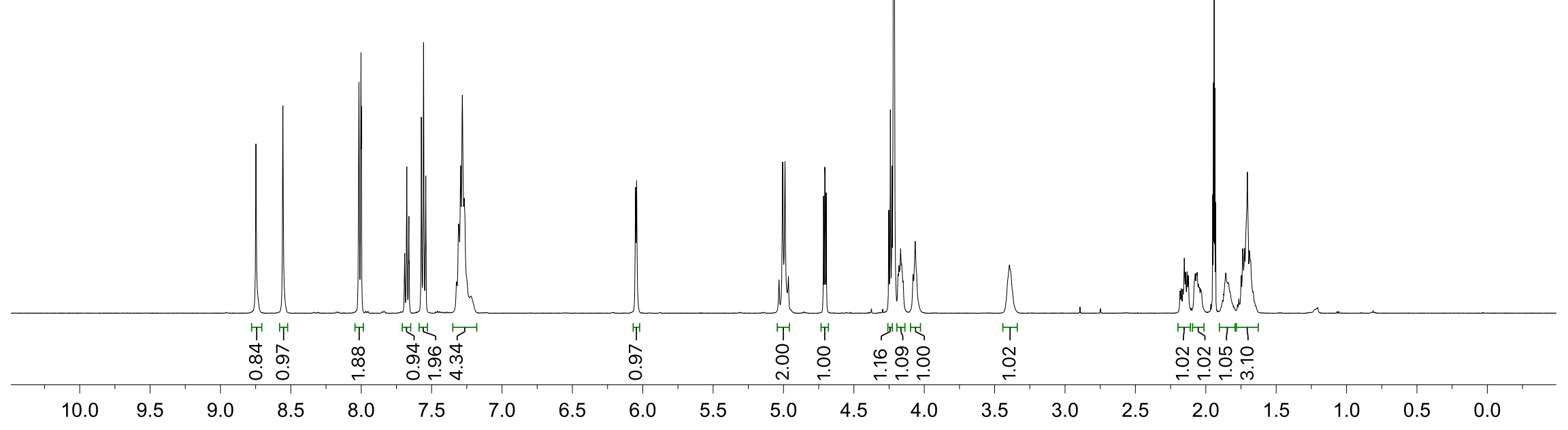




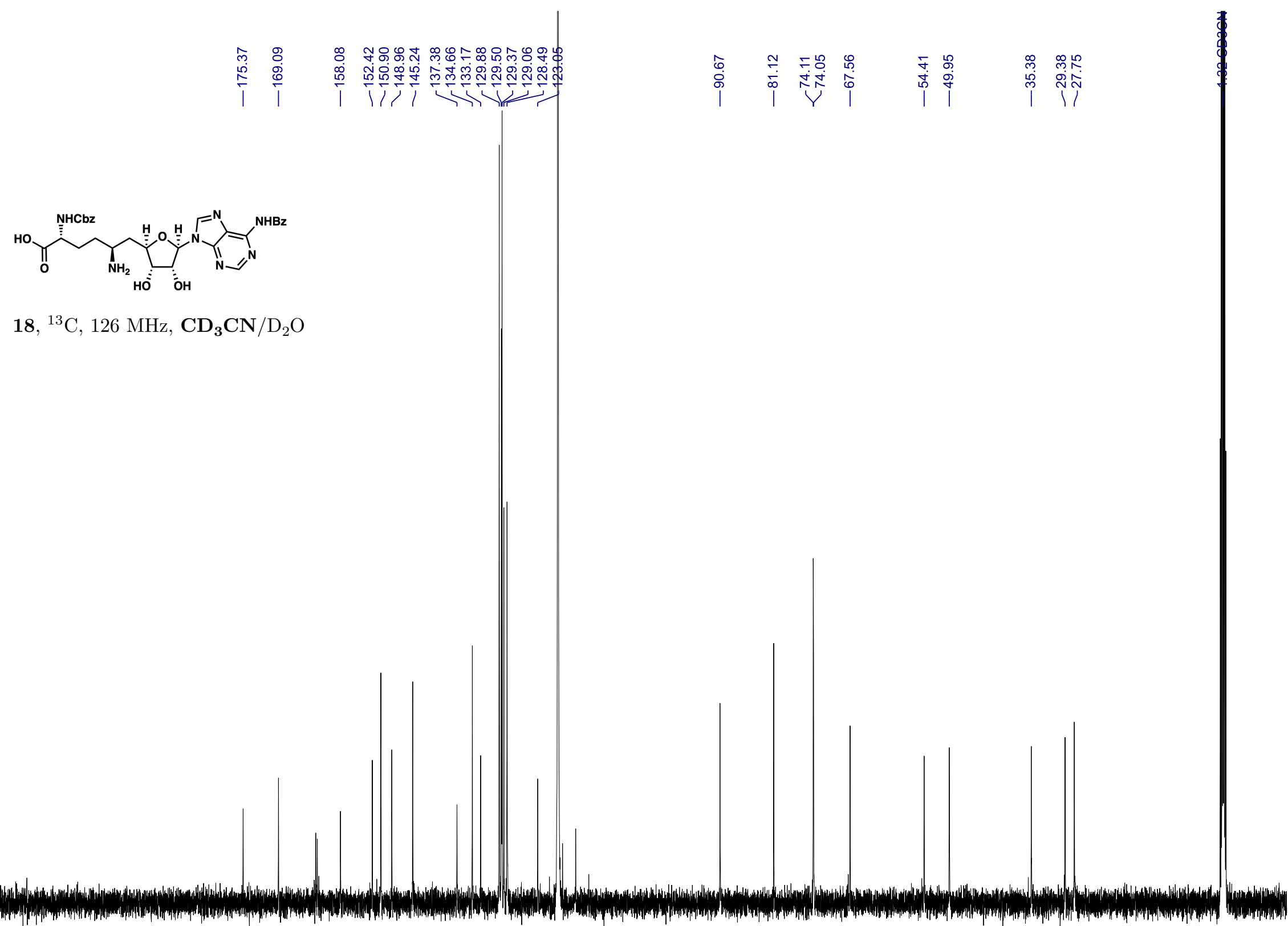




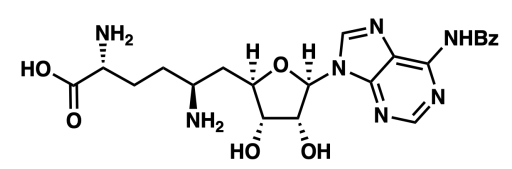

S1, ${ }^{1} \mathrm{H}, 500 \mathrm{MHz}, \mathbf{C D}_{3} \mathbf{C N} / \mathrm{D}_{2} \mathrm{O}$

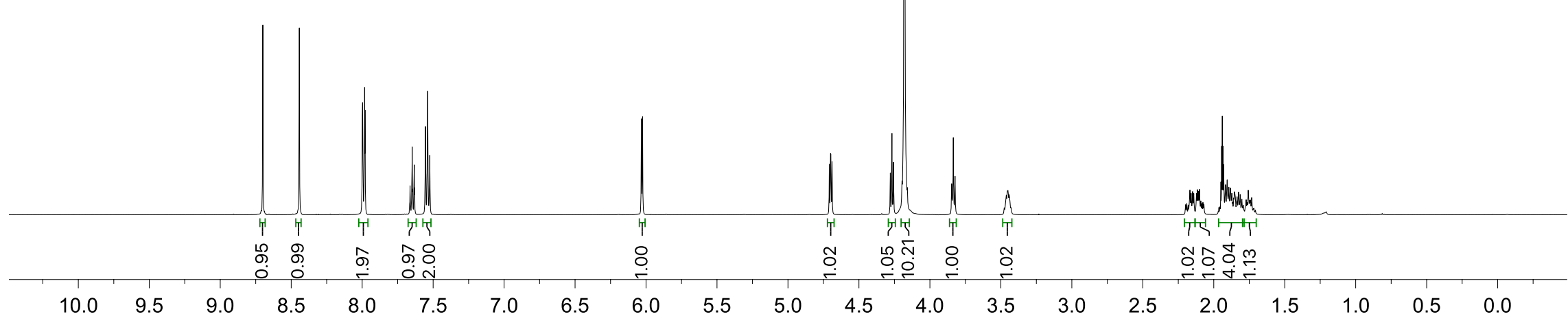




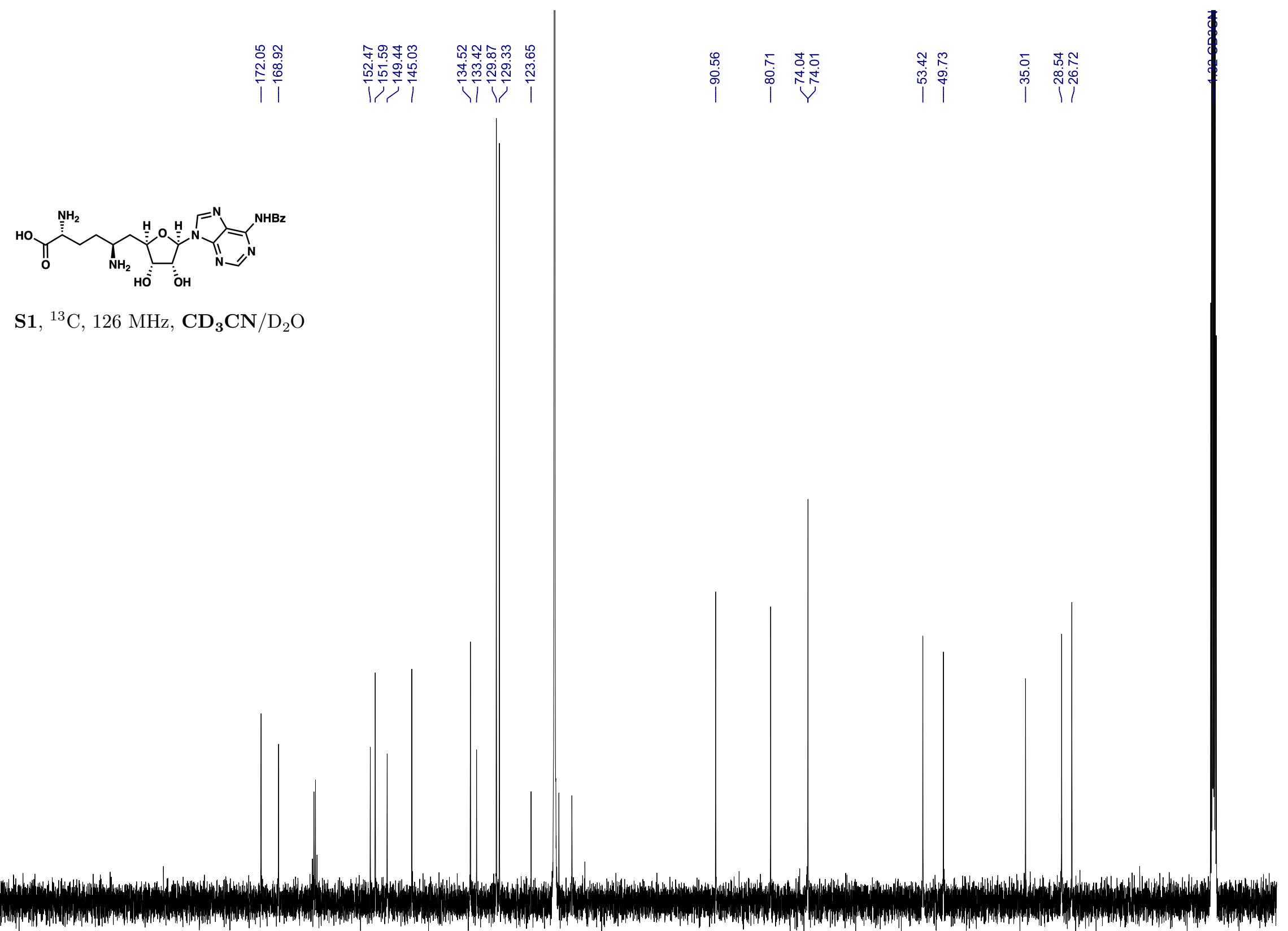

$210 \quad 200$

190

180

$170 \quad 160$

150

130

120

$110 \quad 100$

90

80

60

50

$40 \quad 30$

$20 \quad 10$ 0 


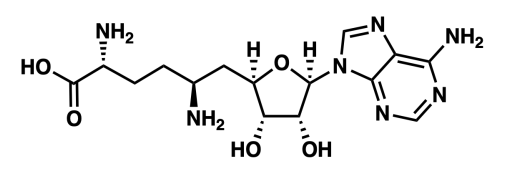

5, ${ }^{1} \mathrm{H}, 500 \mathrm{MHz}, \mathrm{D}_{2} \mathrm{O}$

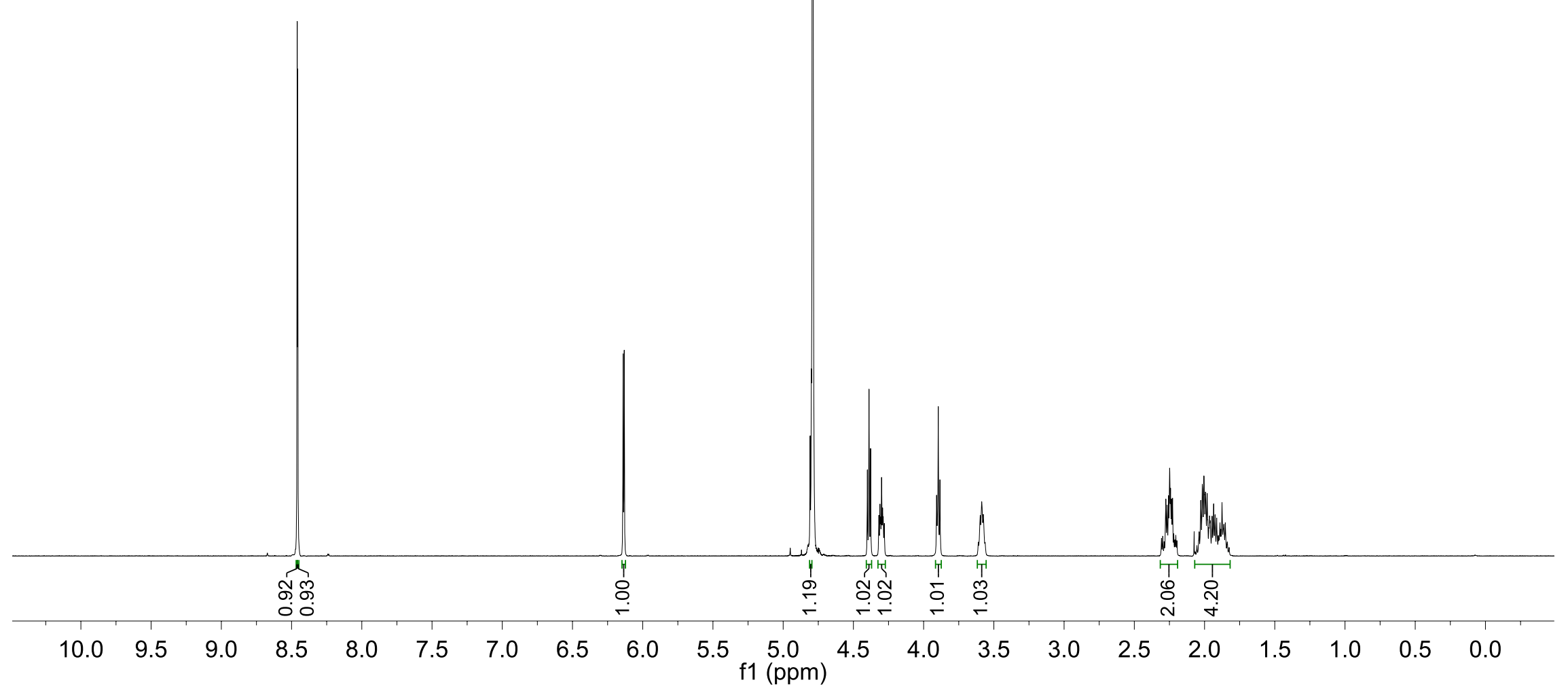




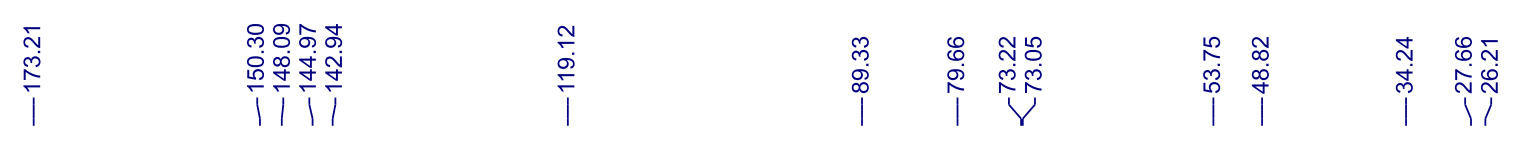

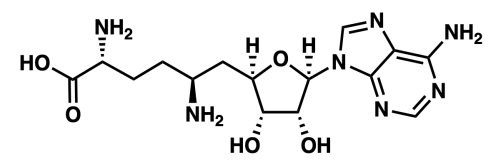

5, ${ }^{13} \mathrm{C}, 126 \mathrm{MHz}, \mathrm{D}_{2} \mathrm{O}$

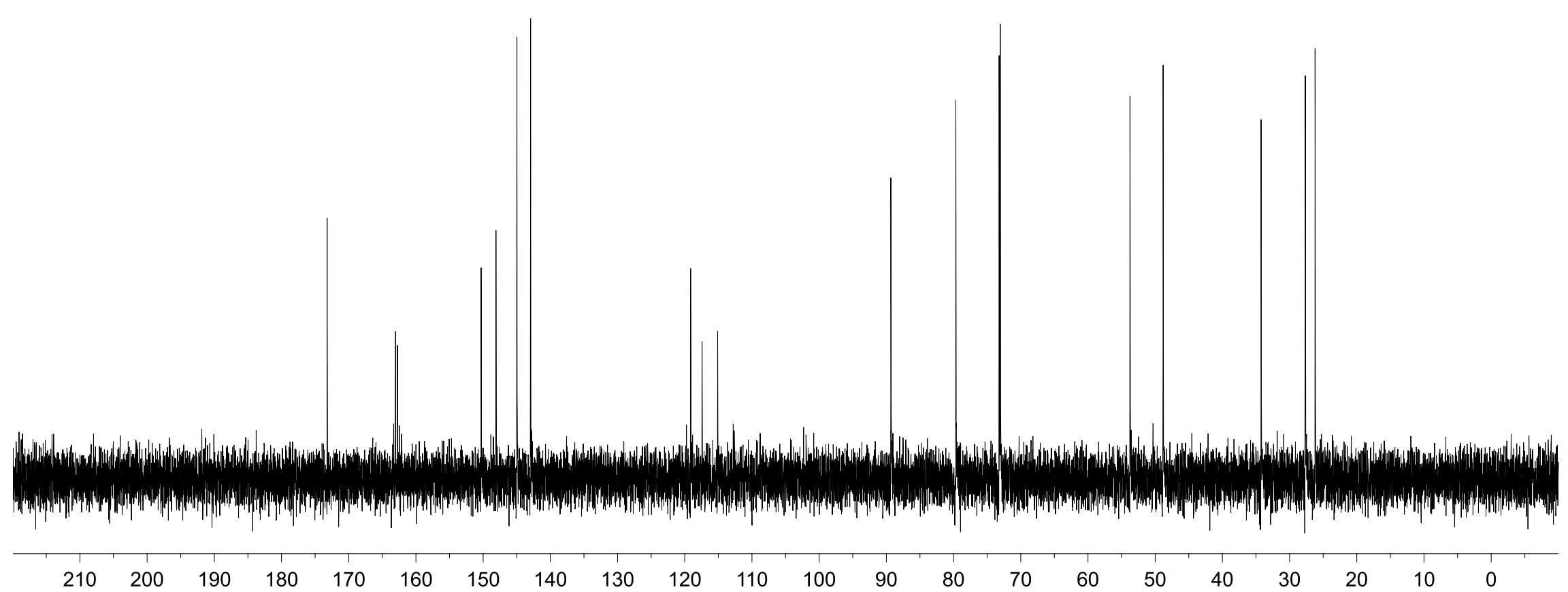




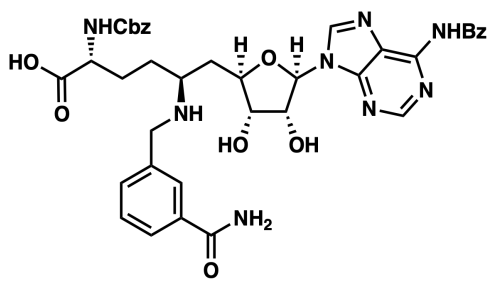

19, ${ }^{1} \mathrm{H}, 600 \mathrm{MHz}, \mathrm{CD}_{3} \mathrm{CN} / \mathrm{D}_{2} \mathrm{O}$

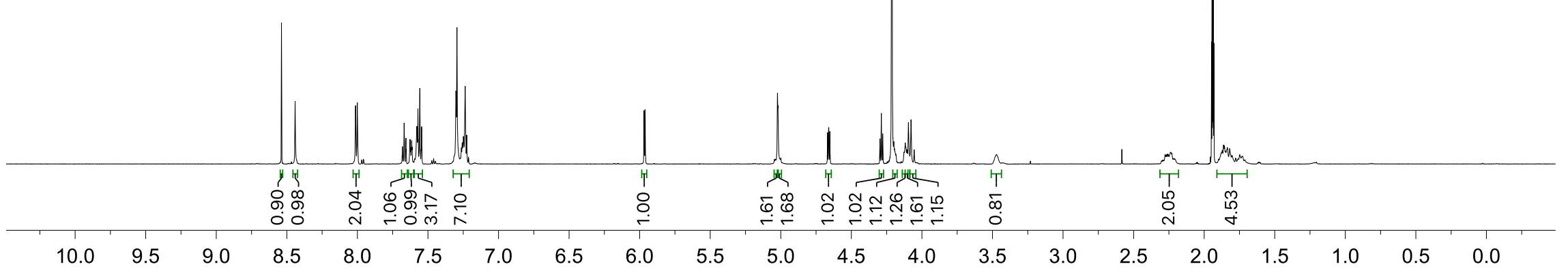




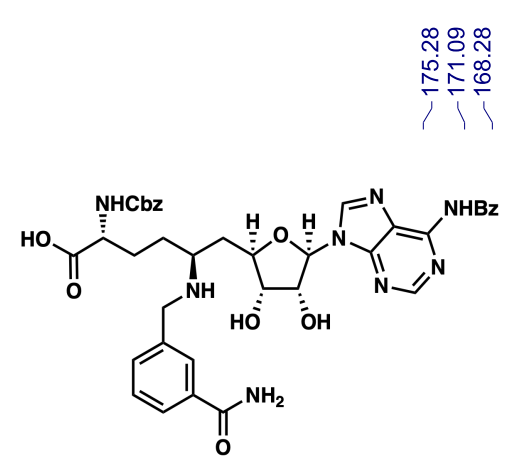

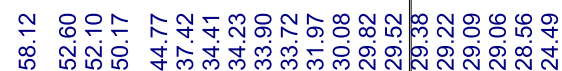

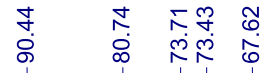

$\stackrel{\infty}{\stackrel{0}{*}} \stackrel{\infty}{\circ}$

$8 \times$

1) ।

กั่งั่

19, ${ }^{13} \mathrm{C}, 126 \mathrm{MHz}, \mathbf{C D}_{3} \mathbf{C N} / \mathrm{D}_{2} \mathrm{O}$

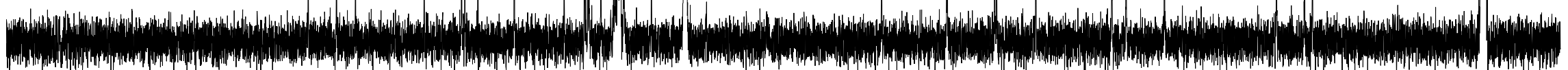

210200

190 
EpiSinefungin_Decultot_Policarpo_ChemRxiv_2020_SI.pdf (6.39 MiB) view on ChemRxiv • download file 
Other files

EpiSinefungin_Xray_Cpd12_ChemRxiv_2020.cif (402.81 KiB)

view on ChemRxiv - download file 